\title{
A review of grass flies (Diptera, Chloropidae) of Karelia and Murmansk Province of Russia
}

\author{
Emilia P. Nartshuk', Alexei V. Polevoi and Andrey A. Przhiboro'
}

\begin{abstract}
Nartshuk EP, Polevoi AV and Przhiboro AA. 2020. A review of grassflies (Diptera, Chloropidae) of Karelia and Murmansk Province of Russia. Fauna Norvegica 40: 47-92.

One hundred and sixteen species of Chloropidae (Diptera) are recorded in Russian Karelia and Murmansk Province: 112 in Karelia and 44 in Murmansk Province. Twenty-two and seven species are new for Karelia and Murmansk Province, respectively. Calamoncosis oscinella is for the first time reported from Russia and Elachiptera breviscutellata - from European Russia. Pseudogaurax venustus is reinstated as Gaurax venustus. Taxonomic notes are provided on Eribolus nana, Polyodaspis ruficornis, Oscinella vindicata and Cetema simile. The distribution and biological data are given for every species. Point maps are provided for species with at least one exactly known location. The zoogeographical structure of Chloropidae fauna on the examined territories is briefly discussed and compared with other north-European countries.
\end{abstract}

doi: 10.5324/fn.v40i0.3406. Received: 2020-02-11. Accepted: 2020-06-01. Published online: 2020-07-02. ISSN: 1891-5396 (electronic).

Keywords: Diptera, Chloropidae, fauna, Karelia, Murmansk Province, Russia.

1. Zoological Institute of Russian Academy of Sciences, Universiterskaya nab. 1, 199034 St Petersburg, Russia 2. Forest Research Institute of Karelian Research Centre of the Russian Academy of Sciences, Pushkinskaya 11, 185910 Petrozavodsk, Russia

Corresponding author: Alexei Polevoi

E-mail: alexei.polevoi@krc.karelia.ru

\section{INTRODUCTION}

The Chloropidae (Diptera, Brachycera, Acalyptratae) is one of the largest acalyptrate families. The family is usually named as grass flies or frit flies. The family is species-rich, nearly 3200 valid species are known in the World (M. von Tschirnhaus pers. comm.) and more than 700 species in the Palaearctic. Chloropidae are distributed worldwide and represented in a great variety of habitats including forests, meadows, wetlands, swamps, bogs, marshes and steppe. The larvae are phytophagous, saprophagous, mycetophagous or carnivorous. The ability of grass-flies to utilize variable substrates made them a family of great ecological importance. Phytophagous larvae develop in the shoots and seeds of Poaceae or in the stems of other plants. Some species in the genera Oscinella, Chlorops, Lasiosina, Meromyza, and Dicraeus are economically important pests of cereals. Saprophagous larvae live in the plant tissues previously damaged by other insects, in fungi or decaying wood with mycelia, in bird nests, in excrements and animal corpses. Carnivorous larvae develop in the egg cocoons of spiders (Araneae) or egg pods of grasshoppers, locusts (Acrididae), mantids (Mantidae), or egg masses of whip spiders (Amblypygi) and dobsonflies (Megaloptera). A few species are predatory on root aphids (Pemphigidae) or thrips (Thysanoptera). Ferrar (1987) and Nartshuk (2014) give extended reviews of larval biology of Chloropidae. Due to species richness and rather simple methods of collecting (sweep net, Malaise traps, color plates), this family is an excellent choice to monitor biodiversity in most terrestrial habitats.
The status of grass flies as a bioindicator group is questionable. It has been demonstrated that they are insensitive to fluorine- and sulphurcontaining aerial emissions (Kozlov \& Zvereva 1997), however, some authors mentioned increasing density of herbivorous flies including Chloropidae in polluted areas (Dabrowska-Prot 1984; Bährmann 1985). Chloropids are among the commonest flies in the examined regions and are found in almost all habitats.

The first list of Karelian Chloropidae containing 17 species dates to the mid-19th century (Chydenius \& Furuhjelm 1859). Frey (1934) recorded 29 species from Karelia and 17 species from Murmansk Province. Kanervo (1942) reported Oscinella frit (Linnaeus 1758) and Chlorops pumilionis (Bjerkander 1778) as pests of cereals in Karelia. Znamenskaya $(1941,1962)$ recorded Oscinella frit also from Murmansk Province. Krogerus (1960) recorded 12 species identified by Frey from bogs in the biogeographical province Regio Kuusamoensis (Paanajärvi and environs), with most of localities situated along the modern Russian-Finnish border or in Russia. Species from both Karelia and Murmansk Province are mentioned in several works (Nartshuk 1998, 1999a; Nartshuk \& Przhiboro, 2003). Additional data on Karelian Chloropidae can be found in numerous local lists (Polevoi 1997, 2006; Yakovlev et al. 2000; Polevoi \& Humala 2003, 2005, 2007, 2009; Humala \& Polevoi 2006, 2008, 2009; Jakovlev et al. 2014). Two species in the genera Chlorops and Incertella have been described based on the materials from Russian Karelia (Duda 1933; Nartshuk \& Przhiboro 2009). Up to now, no 
comprehensive compilation is available for both regions. Eighty-seven species were listed from Karelia and 32 species, from Murmansk Province, in the recent review of North European fauna (Nartshuk \& Andersson 2013), however, exact localities were given only for a few species. In the present work, we give a detailed annotated list of Chloropidae for Murmansk Province and Russian Karelia based on our original material, the material of other authors kept in the collections and the published data, and briefly discuss the features of their distribution in this region.

\section{MATERIAL AND METHODS}

Most of the material examined was collected within the modern borders of administrative regions of Russia: Murmansk Province and the Republic of Karelia. In several cases, we also give additional data from neighboring areas of Leningrad and Archangelsk provinces. This area covers the entire Russian part of Fennoscandia, except the Karelian Isthmus. As finer delimitation, we use the biogeographical provinces of Fennoscandia so that our data are compliant with Scandinavian literature. Province borders and abbreviations (Figure 1) follow Henkinheimo \& Raatikainen (1971) and Ahti \& Boychuk (2006).

The list of species is based on the materials from the following collections: Zoological Institute of the Russian Academy of Sciences, St Petersburg, Russia (ZISP), Natural History Museum, Helsinki, Finland (NHMH) and Forest Research Institute, Russian Academy of Sciences, Petrozavodsk, Russia (FRIP). All species were identified or verified by the first author. The species listed by Frey (1934) were reexamined.

Systematics and nomenclature follow Ismay \& Nartshuk (2000). Nomenclature of plant species is given according to Kravchenko (2007). Regions and localities are listed from north to south and from west to east. In the list, we provide only modern locality names, while the information on older names (or names used on the labels) and geographical coordinates are given separately (Appendix 1). Published records are mentioned only in the cases when the original materials from appropriate localities (or regions) were not seen or a species was published under a different name. There is a problem with the data by Rolf Krogerus from the biogeographic province Regio Kuusamoensis (Krogerus 1960), as his records may refer to Karelia, Murmansk Province or Finland. Pending future revision, we provisionally list these materials as being collected in Karelia, Paanajärvi.

The zoogeographical analysis was based on available checklists of North European countries (Stackelberg 1958; Nartshuk 1962; Nartshuk \& Elberg 1979; Chandler 1998; Wendt 1999; Karpa 2001; Petersen 2001; Zatwarnicki 2001; Pakalniškis et al. 2006; Nartshuk \& Andersson 2013; Nartshuk \& Kahanpää 2014). We have also included later additions for Poland (Grochowska 2006, 2007), Denmark (Nielsen 2014, 2015) and Finland (Haarto et al. 2019), as well as unpublished data available from online resources (http://dipteratyoryhma.myspecies.info/fi/content/checklist-errataaddenda; https:/artskart.artsdatabanken.no) or received from personal communications by Michael von Tschirnhaus. We used nonmetric multidimensional scaling (NMDS) to analyse the similarity of the faunas. It was performed with freely available PAST software (Hammer et al. 2001), using the Dice similarity measure. Maps were produced using QGIS (https://qgis.org). Underlying map layers are based on Open Street Map and Natural Earth data (https://www. naturalearthdata.com; https://www.openstreetmap.org), except for the

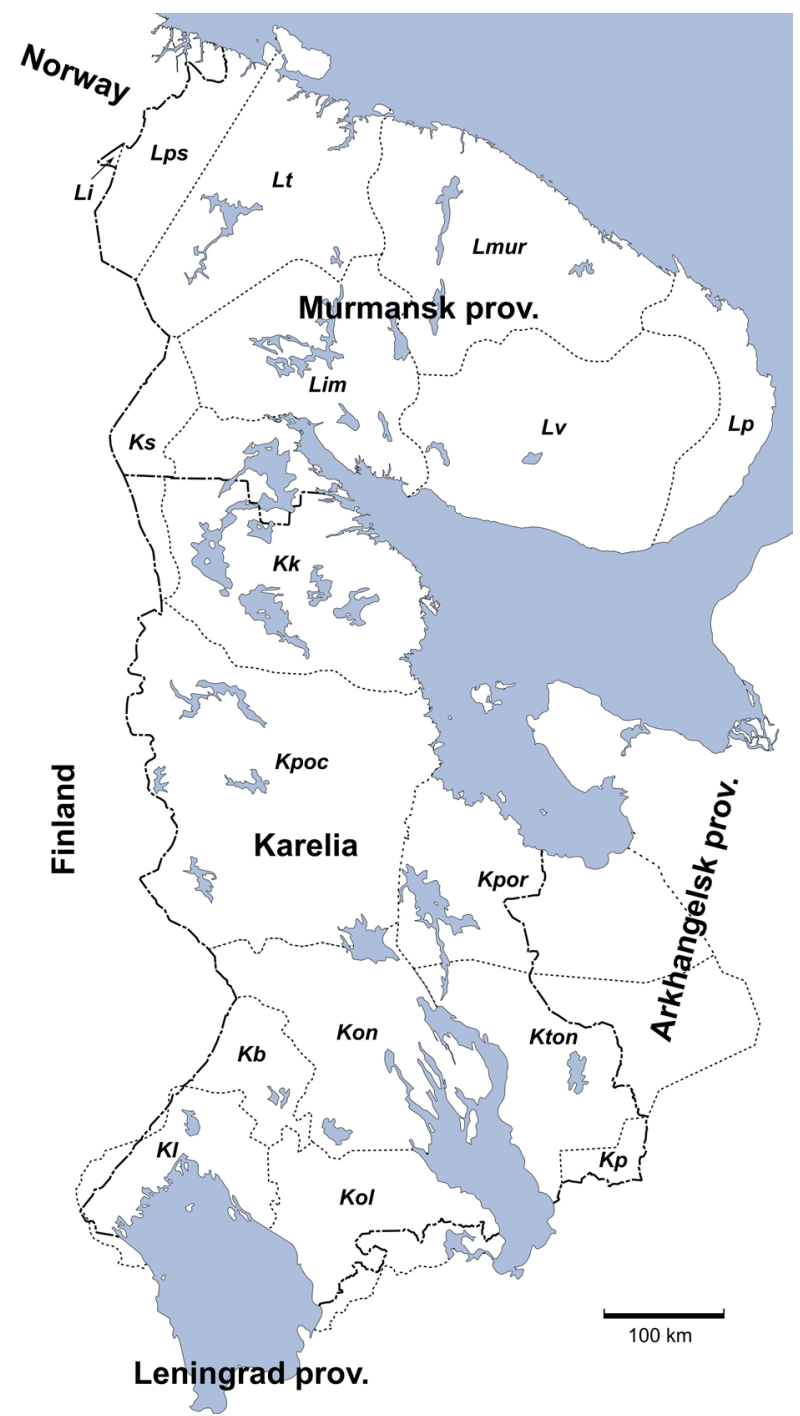

Figure I. Delimitation of biogeographical provinces in the Russian part of Fennoscandia. Li - Lapponia inarensis, Lps - Lapponia petsamoensis, Lt - Lapponia tulomensis, Lmur - Lapponia murmanica, Lp - Lapponia ponoensis, Lim - Lapponia Imandrae, Lv - Lapponia Varsugae, Ks Regio kuusamoensis, Kk - Karelia keretina, Kpoc - Karelia pomorica occidentalis, Kpor - Karelia pomorica orientalis, Kb-Karelia borealis, Kon - Karelia onegensis, Kton - Karelia transonegensis, Kl - Karelia ladogensis, Kol-Karelia olonetsensis, Kp - Karelia pudogensis.

borders of biogeographical provinces, which were manually digitized by the authors.

\section{Species List}

The list contains 116 species recorded from Russian Karelia (112 species) and Murmansk Province (44 species). Species new for Karelia are marked with a single asterisk $\left(^{*}\right)$ and those for Murmansk Province, with a double asterisk (**). Distribution maps are given in Appendix 2.

\section{Subfamily Oscinellinae}

Aphanotrigonum cinctellum (Zetterstedt, 1848).

Material examined: Karelia. Kol: 1 female, Petrozavodsk (NHMH). Distribution: Widely distributed in the Palaearctic. Tschirnhaus 
(1981) recorded this species as A. fasciellum (Zetterstedt 1855) from the Afrotropical region (mangrove flowers in Gambia).

Biological notes: Dry habitats and coastal salt marshes, in which it is the second abundant chloropid after Oscinimorpha albisetosa (Duda 1932) along an investigated German coast section along the North Sea (Tschirnhaus 1981). Adults were reared from different species of Poaceae.

\section{Aphanotrigonum nigripes (Zetterstedt, 1848)}

Material examined: Karelia. Ks: 1 female, Paanajärvi, Frey (NHMH); Kk: 23 males, 7 females, Kartesh, 9-16.VIII.1989, Sugonyaev; 20-21. VIII.1996, 3-5.VII.2000, 12-20.VI.2002, Przhiboro (ZISP); Kpor: 1 male, Island Kondostrov, 20.VIII.2002, Humala (FRIP); Kon: 1 male, Vegoruksy, 24-29.VI.2013, Polevoi (FRIP); Kol: 1 male, Lososinnoe, 28-31.V.2013, Polevoi (FRIP); 1 female, Petrozavodsk, Frey (NHMH). Published records: Karelia. Kol: Petrozavodsk (Frey 1934, as Conioscinella cinctella Zetterstedt, 1848; misidentification).

Distribution: Holarctic boreal species.

Biological notes: Meadows and wetlands, including the sea intertidal and supralittoral zones. In pitfall traps of a dune ecosystem in Denmark (Nielsen et al. 2019) it was the second abundant chloropid after Conioscinella zetterstedti Andersson 1966. Larvae develop in stems of plants damaged by other insects. Hibernate as adults. Adults were collected on and reared from stems of cereals.

\section{Aphanotrigonum trilineatum (Meigen, 1830)}

Material examined: Murmansk Province. Lim: 1 female, Kandalaksha (NHMH). Karelia. Ks: 2 males, 2 females, Paanajärvi, Frey (NHMH); Kon: 1 male, Island Bukol'nikov, 25.VI.2003, Polevoi (FRIP); Kol: 1 female, Lososinnoe, 28-31.V.2013, Polevoi (FRIP); 3 males, 2 females, Petrozavodsk; 1 female, Sheltozero, 29.VIII.1942, Tiensuu (NHMH).

Distribution: Holarctic boreal species.

Biological notes: Meadows and wetlands, sometimes agricultural fields. Larvae develop in stems of Poaceae including plants damaged by other insects. Hibernate as adults.

\section{*Calamoncosis aprica (Meigen, 1830)}

Material examined: Karelia. Kon: 1 female, Gomsel'ga, 9.VIII.2018, Polevoi (FRIP).

Distribution: European species.

Biological notes: Wet meadows and wetlands. Larvae phytophagous or phytosaprophagous, living as inquilines in stems of Phragmites australis (Cav.) Trin. ex Steud. damaged by larvae of Platycephala planifrons (Fabricius 1798) or rarely by caterpillars of Archanara geminipuncta (Haworth 1809) (Lepidoptera, Noctuidae) (Tschirnhaus 1981; Grochowska 2002). Grochowska (2002) studied the life cycle of the species in Poland.

\section{Calamoncosis glyceriae Nartshuk, 1958}

Published records: Karelia. Without locality name (Nartshuk \& Andersson 2013).

Distribution: Eurasian species.
Biological notes: Wet habitats near lakes and rivers. Larvae phytophagous, feed gregariously in unsheathed inflorescences of Glyceria spp.

\section{*Calamoncosis oscinella (Becker, 1910)}

Material examined: Karelia. Kon: 1 female, Gomsel'ga, 6.VI.2013, Polevoi; 1 female, Polya, 26.VI.2013, Polevoi (FRIP).

Distribution: Rare European species. Few specimens are known from Finland, Sweden, Norway and Germany. This is the first record from Russia.

\section{Conioscinella frontella (Fallén, 1820)}

Material examined: Murmansk Province. Lps: 1 male, Trifonovo, 1 female, Pechenga (NHMH); Lim: 2 females, Lake Bolshoi Vudjavr, 13.VIII.1931, Fridolin; 21.VIII.1931, Belyakova; (ZISP). Karelia. Kk: 1 female, Kartesh, 22.VII.1975, Gorodkov (ZISP); Kon: 2 females, Semchezero (NHMH); 1 male, Nizhnee Myagrozero, 20-24.VII.2012, Polevoi; Kl: 1 male, Valaam, 31.VII.2009, Polevoi; Kol: 1 male, Lososinnoe, 28-31.V.2013, Polevoi (FRIP).

Published records: Murmansk Province. Li: Kola (Frey 1934).

Distribution: Widely distributed in the Palaearctic, ranges from Europe to Mongolia and Israel.

Biological notes: Meadows and agricultural fields. Larvae phytophagous, develop in different species of Poaceae, hibernate in the basal tillers of grasses and pupate next spring. Kleptoparasite of predaceous insects and spiders (Tschirnhaus et al. 2015).

\section{*Conioscinella gallarum (Duda, 1933)}

Material examined: Karelia. Kl: 5 males, Harlu, 9-10.VI.1933, reared from Cirsium inflorescences, Tiensuu (NHMH).

Distribution: Euro-Caucasian species. Nartshuk \& Andersson (2013) mention Karelia in the "Biology" section, but do not indicate it formally in the catalogue. We hence consider this species new for the region.

Biological notes: The species was earlier reared from galls of Andricus sp. and Biorhiza pallida Olivier 1791 (Hymenoptera, Cynipidae) on Quercus, from cones of Larix decidua Mill., buds of Populus tremula L. and inflorescences of Cirsium (Duda 1933; Nartshuk \& Andersson 2013).

\section{Conioscinella livida Nartshuk, 1970.}

Material examined: Murmansk Province. Lps: 1 female, Yläluostari, Hellen; 1 male, Pechenga; 1 male, Lotta River, Platonoff (NHMH). Distribution: European species, known from Estonia, Leningrad Province and Germany (Bavaria).

Biological notes: Bogs.

\section{*Conioscinella mimula Collin, 1946}

Material examined: Karelia. Kon: 1 female, Island Radkol'e, 30.VII.2018, Polevoi (FRIP).

Distribution: Eurasian species. 
Biological notes: Meadows. Larvae phytophagous, live in shoots of Anthoxanthum odoratum L., Bromus sp., Lolium perenne L., hibernate in shoots of host plant and pupate in the next spring (Nye 1958, 1959; Tschirnhaus 1981).

\section{Conioscinella sordidella (Zetterstedt, 1848)}

Material examined: Karelia. Kk: 1 female, Syrovatka, 16.VII.2003, Polevoi (FRIP).

Distribution: European species.

Biological notes: Dry meadows. Larvae phytophagous, live in shoots of Poaceae.

\section{Conioscinella zetterstedti Andersson, 1966}

Material examined: Karelia. $K l: 1$ female, Sortavala (NHMH). Published records: Karelia. Kl: Kirjavalahti (Frey 1934, as C. brachyptera Zetterstedt 1848; misidentification).

Distribution: Holarctic species.

Biological notes: Inland and coastal dunes or sandy beaches, among grasses and sedges. The most abundant chloropid in pitfall traps in Danish dunes (Nielsen et al. 2019). Flies are wingless or brachypterous, but the size of wings and halteres is variable (Brauns 1938). Flies are excellent jumpers and run rapidly on plants and on the sand surface. Larvae phytophagous in shoots of Poaceae.

\section{Dicraeus fennicus Duda, 1933}

Material examined: Karelia. Kk: 1 male, Gridino, 14.VII.2007, Humala (FRIP); 1 female, Kartesh, 16-29.VII.1966, Tanasijtshuk; 1 male, Island Sidorov, 23-24.VII.1966, Tanasijtshuk (ZISP); Kpoc: 1 male, Island Nemetskii Kuzov, 17.VII.2001, Humala; 1 male, Island Russkii Kuzov, 18.VII.2001, Humala; Kpor: 1 male, Island Pechak, 24.VII.2001, Humala (FRIP); Kon: 1 male, Konchezero, 6.VII.1942, Tiensuu (NHMH); 1 female, Kurgenitsy, 18.VII.2000, Polevoi; Kl: 1 female, Kirjavalahti, 4.VII.2005, Polevoi (FRIP).

Distribution: Holarctic species.

Biological notes: Meadows. Larvae phytophagous, develop in unripe seeds of Elymus spp., more commonly of E. repens (L.) Gould.

\section{Dicraeus rossicus Stackelberg, 1955}

Material examined: Murmansk Province. Lim: 1 female, Kandalaksha (NHMH). Karelia. Kk: 1 male, Sonostrov (NHMH); Kpoc: 1 male, 1 female, Island Nemetskii Kuzov, 17.VII.2001, Humala (FRIP).

Distribution: Transpalaearctic species, distributed from Europe to Japan.

Biological notes: Meadows. Larvae phytophagous, feed in developing seeds of Elymus caninus (L.) L.

\section{Dicraeus tibialis (Macquart, 1835)}

Published records: Murmansk Province. Lim: Kandalaksha (Frey 1934, as D. pallidiventris Macquart, 1835). Karelia. Without locality name (Nartshuk \& Andersson 2013); Kl: Kirjavalahti (Frey 1934, as
D. pallidiventris).

Distribution: Holarctic species, but recently found in New Zealand (Ismay 1991).

Biological notes: Meadows. Larvae phytophagous, feed in developing seeds of Bromopsis spp., Helictotrichon pubescens (Huds.) Pilg. and probably in other grasses.

\section{** Elachiptera breviscutellata Nartshuk, 1964}

Material examined: Murmansk Province. Lim: 1 male, 3 females, Island Berezhnoi Vlasov, 15-16.VIII.1993, Przhiboro (ZISP).

Distribution: New record for European Russia and northern Europe. The species is known from Austria, Germany, Hungary, Lithuania, Asian Russia (West Siberia), Kazakhstan and Mongolia (Nartshuk 1984).

Biological notes: Our specimens were collected in the supralittoral zone of the White sea shore (meadow with halophytic vegetation). Tschirnhaus (1981) caught it from May to October in yellow pan traps in salt-influenced Phragmites stands in Germany.

\section{Elachiptera cornuta (Fallén, 1820), sensu lato}

Taxonomic notes: Here, we consider the species in the broad sense, including the specimens with thin and broad arista and with some variability in the male genitalia.

Material examined: Murmansk Province. Lps: 3 females, Pechenga (NHMH); Lim: 1 female, Lake Vudjarv, 19.VI.1930, Fridolin (ZISP); 1 male, 3 females, Kandalaksha (NHMH); 1 male, Kolvitsa, 9.VIII.1995, Gorodkov (ZISP). Karelia. Kk: 1 female, Vartolambina, 22.VI.1998, Polevoi(FRIP); 7 males, 5 females, Primorskii, 17.VIII.1996, Przhiboro; 1 male, Chupa, 30.VI.2000, Przhiboro; 35 males, 43 females, Kartesh, 9-16.VIII.1989, Sugonyaev; 15.VII-21.VIII.1996, 5.VII.1997, 3-20. VII.2000, 12.VI.2002, 20.VI.2002, 4.IX.2005, Przhiboro; 22-26. VII.2010, Nartshuk; 1 pupa (reared to adult), Kartesh, 25.VIII.1996, Przhiboro; 1 male, Nikol'skaya Bay, 28.VII.1996, Przhiboro (ZISP); 3 females, Island Malyi Andronin, 20.VII.2010, Nartshuk (ZISP); 1 female, Gridino, 9.VIII.2006, Polevoi; 1 female, Gridino, cape Purnavolok, 7.VIII.2007, Humala; 1 female, Island Pezhostrov, southern shore, 7.VIII.2006, Polevoi; Kpoc: 1 female, Island Nemetskii Kuzov, 22.VIII.2002, Humala; 1 male, Belomorsk, 10.VIII.2018, Grichanov; Kpor: 1 female, Island Kondostrov, 20.VIII.2002, Humala; 1 male, Segezha, 5.VI.2019, Polevoi (FRIP); Kon: 1 male, 1 female, Shaidoma, 10-11.VIII.2018, Polevoi; 1 female, Girvas, 7.IX.2003, Polevoi; 2 females, Belaya Gora, 7.IX.2003, Polevoi (FRIP); 5 males, 5 females, Kivach, 30.IX.1923, Fridolin (ZISP); 22-26.X.1990, Kutenkova, 15.V.1997, 3.IX.2002, 27.V-31.VII.2003, 25.IV-23.VI.2016, Polevoi; 1 female, Myagrozero, 5.IX.2003, Polevoi; 1 female, Pod'el'niki, 22.VII.2011, Polevoi; 1 female, Island Bukol'nikov, 30.VII.2018, Polevoi; 2 females, Island Bol'shoi Lelikovskii 31.VII.2001, Polevoi; 1 female, Lelikovo, 26.VI.2003, Polevoi; 2 females, Island Lyudskoi, 24.VI.2003, Polevoi; 1 male, 1 female, Island Bukol'nikov, 25.VI.2003, Polevoi; 1 female, Island Eglov, 26.VI.2014, Polevoi; 1 female, Island Ernitskii, 26.VI.2003, Polevoi (FRIP); Kpoc: 1 female, Lake Kuzharvi, 21.VI.2000, Polevoi (FRIP); Kl: 1 female, Island Kilpola, 12-17. VI.2011, Polevoi; 4 males, 2 females, Puikkola, 15.V-29.IX.1991, Polevoi (FRIP); 1 female, Sortavala, 11.VI.1933, Frey (NHMH); Kol: 1 female, Vidlitsa, 3.IX.2018, Polevoi (FRIP); Kton: 2 females, Shoikapolda River, 22.VIII.2006, Polevoi; 1 male, Sukhaya Vodla River, 7.VI.2002, Polevoi; 1 female, Koskosalma, 7.VI.2002, Polevoi 
(FRIP); Kp: 1 female, Ust'-Reka, 23.VI.2009, Humala; 1 male, Korbozero, 22.VI.1996, Polevoi (FRIP).

Published records: Karelia. Ks: Paanajärvi (Krogerus 1960).

Distribution: Transpalaearctic polyzonal species.

Biological notes: Meadows and wetlands, including the White sea intertidal and supralittoral zone, also agricultural fields. Larvae saprophytophagous, secondary invaders, develop in rotting tissues of plants: Poaceae including cereals, Cyperaceae and some other monoand dicotyledons damaged by other insects. Hibernate as adults, e.g. under litter, in empty Lipara galls, plant stems or old bird nests.

\section{**Elachiptera diastema Collin, 1946}

Material examined: Murmansk Province. Lim: 1 female, Laplandskii Nature Reserve, Vtoroi Stream, 29.V.2014, Polevoi (FRIP); 1 male, Umba, 13.VIII.1995, Gorodkov (ZISP). Karelia. Kk: 2 males, 1 female, Kartesh, 4.VIII.1996, 8.VII.1997, 4.IX.2005, Przhiboro (ZISP); 1 female, Gridino, 9.VIII.2006, Polevoi; Kpoc: 1 female, Island Russkii Kuzov, 19.VII.2001, Humala; Kon: 1 female, Kivach, 15.V.1997, Polevoi; 1 female, Kurgenitsy, 18.VII.2000, Polevoi; 1 male, Gomsel'ga, 4-6.VII.2012, Polevoi; 1 male, Pin'guba, 17.VI.2007, Polevoi; Kton: 1 male, Sukhaya Vodla River, 24.VIII.2006, Polevoi (FRIP).

Distribution: Euro-Mediterranean polyzonal species.

Biological notes: Meadows and agricultural fields. Larvae phytosaprophagous, develop in stems of plants damaged by other insects. Hibernate as adults.

\section{*Elachiptera scrobiculata (Strobl, 1901)}

Material examined: Karelia. Kk: 1 female, Island Malyi Andronin, 20.VII.2010, Nartshuk (ZISP); Kol: 2 females, Lososinnoe, 28-31.V.2013, Polevoi (FRIP).

Distribution: Transpalaearctic boreal species, distributed from the British Islands to the Far East of Russia, but not common everywhere. Biological notes: Wet habitats with sedges.

\section{Elachiptera tuberculifera (Corti, 1909)}

Material examined: Karelia. Kk: 6 males, 8 females, Kartesh, 9-16.VIII.1989, Sugonyaev; 21.VIII.1996, 6-25.VII.2000, 12.VI.2002, Przhiboro; 22-26.VII.2010, Nartshuk; 4 males, 4 females, Island Malyi Andronin, 29.VII.2010, Nartshuk (ZISP); 1 male, Chupa, 30.VI.2000, Przhiboro (ZISP); Kpor: 1 male, Segezha, 5.VI.2019, Polevoi (FRIP); Kon: 3 females, Shaidoma, 10-11.VIII.2018, Polevoi; 1 male, 2 females, Girvas, 7.IX.2003, Polevoi; 2 males, 1 female, Kivach, 27.V.2003, Polevoi; 1 female, Kosalma, 10.IX.2003, Polevoi; 1 female, Island Lyudskoi, 24.VI.2003, Polevoi; 2 females, Lelikovo, 24.VI.2003, Polevoi; 1 female, Myagrozero, 5.IX.2003, Polevoi; 2 males, Nizhnee Myagrozero, 20-24.VII.2012, Polevoi (FRIP); 1 female, Konchezero (NHMH); Kol: 1 male, Petrozavodsk; 2 females, Sheltozero (NHMH); 1 male, 1 female, Mayachino, 25.VI.2012, Polevoi (ZISP); Kl: 1 male, Impilahti (NHMH); 1 female, Niemelänhovi, 16.VI.2015, Polevoi (FRIP); Kp: 1 female, Korbozero, 22.VI.1996, Polevoi (FRIP); Kton: 2 males, Sukhaya Vodla River, 7-8.VI.2002, Polevoi (FRIP); Leningrad Province: Kol: 1 female, Gumbaritsy (NHMH).

Published records: Murmansk Province. Kola Peninsula, without locality name (Nartshuk \& Andersson 2013).
Distribution: Transpalaearctic polyzonal species.

Biological notes: Wetlands and meadows, including the White sea intertidal and supralittoral zone. Larvae phytosaprophagous, develop in decaying plant tissues; secondary invaders of grasses including cereals, also in stems of Liliaceae, Iridaceae and Typhaceae damaged by other insects. Hibernate as adults and occurs as adults already in the earliest spring time.

\section{*Eribolus hungaricus Becker, 1910}

Material examined: Karelia. Kk: 1 female, Kartesh, 18.VI.1997, Przhiboro (ZISP).

Distribution: European species; known from southern Fennoscandia (Denmark, southern provinces of Finland, Sweden and Norway) (Nartshuk \& Andersson 2013). Our record extends the species range far north.

Biological notes: Our specimen was collected in the upper intertidal zone of the White sea shore (meadow with halophytic vegetation).

\section{Eribolus nana (Zetterstedt, 1838)}

Taxonomic notes: Here we retain the species name according to the International Codex of Zoological Nomenclature (Art. 31.2.2) and following Sabrosky (1967), who considered specific epithet nana in Oscinis nana Zetterstedt, 1838 as a noun.

Material examined: Karelia. Ks: 1 male, 3 females, Paanajärvi, Frey (NHMH); Kon: 1 female, Island Bukol'nikov, 25.VI.2003, Polevoi (FRIP); 1 male, Konchezero, 19.VI.1936, Frey (NHMH); Kl: 1 female, Sortavala, Hellen (NHMH); 1 female, Ladoga, Nordquist (NHMH). Published record: Leningrad Province. Kol: Svir' (Frey 1934, as E. sudeticus Becker, 1910).

Distribution: Holarctic species, widely distributed in the Palaearctic. Biological notes: Wetlands, especially those with reeds. Larvae develop in shoots and stems of hygrophilous monocotyledons damaged by other insects.

\section{*Eribolus slesvicensis Becker, 1910}

Material examined: Karelia. Kk: 5 males, 11 females, Kartesh, 15.VII.1996, 18.VI.1997, 2.VII.1997, Przhiboro (ZISP).

Distribution: Eurasian species, eastward to Mongolia. In Fennoscandia, it was recorded only from few southern provinces of Sweden and Norway (Nartshuk \& Andersson 2013). Our record extends the species range far north.

Biological notes: Wetlands, associated with reed beds (Phragmites australis) especially at the sea and lake shores. Tschirnhaus (1981) listed Carex and Bolboschoenus maritimus (L.) Palla as host plants. Ismay (1980) recorded the species from saline habitats in England. Our specimens were collected only in Phragmites stands at the White sea shore (upper intertidal zone).

\section{Gaurax borealis (Duda, 1933)}

Material examined: Karelia. Ks: 1 female, Paanajärvi, Frey (NHMH).

Published record: Karelia. Ks: Paanajärvi (Frey 1934, as Tropidoscinis gallarum Duda, 1933; misidentification). 
Distribution: Eurasian species, distributed from Europe to Mongolia. Biological notes: Forests.

\section{**Gaurax dubius (Macquart, 1835)}

Material examined: Murmansk Province. Lim: 1 female, Laplandskii Nature Reserve, Vtoroi Stream, 23.VII-2.VIII.2013, Polevoi (FRIP). Karelia: Kon: 1 female, Lelikovo, 24.VI.2003, Polevoi (FRIP); Kol: 1 female, Kaskesruchei, 19-22.VII.2004, Polevoi (FRIP). Arkhangelsk Province: Kton: 1 female, Vozhosel'ga, 8.VII-2.VIII.2014, Polevoi (FRIP).

Published record: Karelia. Ks: Paanajärvi (Frey 1934).

Distribution: Holarctic species.

Biological notes: Forests. Larvae fungivorous, develop in bracket fungi, including old dry fungi, under bark of trees infested by dendrophilous Coleoptera. Adults also visit fungi.

\section{Gaurax leucarista Nartshuk, 1962}

Material examined: Karelia. Kon: 1 female, Kivach, 12-20.VI.1989, Polevoi (ZISP).

Distribution: European species.

Biological notes: Forests. Collected on young aspens and reared from larvae collected under bark of beech (material by V.G. Kovalev from Moscow Province).

\section{Gaurax venustus Czerny, 1906, stat. rev.}

Taxonomic notes: Kanmiya (1983) transferred this species to the genus Pseudogaurax Malloch, based on the elongated scutellum. Other important characters of Pseudogaurax are as follows: ocellar triangle reaching anterior margin of frons, outer vertical seta much longer than inner vertical seta, first flagellomere obliquely elongate, arista thickened and densely pubescent. With more available materials of $P$. venustus it has become clear that all characters, except the elongated scutellum, correspond well with the genus Gaurax (ocellar triangle reaching about the middle of frons, outer vertical seta subequal to inner vertical seta, first flagellomere rounded or reniform, arista pubescent but not thickened, wing membrane covered by rather long trichiae). We consider this as sufficient basis to reinstate the original combination.

Material examined: Karelia. Kon: 3 females, Kivach, 12.VI-20. VII.1989, Polevoi; 1 male, Kondopoga, 4.VIII.2012, A. Kainelainen (ZISP).

Published records: Karelia. Kon: Kivach (Nartshuk 1999c, as Pseudogaurax venustus (Czerny, 1906)).

Distribution: European species.

Biological notes: Forests.

\section{Hapleginella laevifrons (Loew, 1858)}

Material examined: Murmansk Province. Lim: 1 male, Monchegorsk, 24.VII.1995, Kozlov (ZISP); 1 male, Kandalaksha (NHMH); 1 female, Island Ryashkov, 15.VII.1992 (ZISP). Karelia. Kl: 1 male, Kirjavalahti (NHMH); 1 male, Sortavala (NHMH); Kon: 1 female, Tereki, 21.VII.2017, Polevoi; 1 female, Kivach, 4.VII.2001, Polevoi (FRIP).
Distribution: Transpalaearctic species distributed from the British Isles to the Far East of Russia, also known in Greece (Kailidis \& Georgevits 1972), Morocco (El-Hassani \& Messaoudi 1986) and Portugal (Ebejer \& Andrade 2015).

Biological notes: Forests. Larvae develop in cones of different coniferous trees (Pinus, Picea, Abies, Larix) infested by other insects and can develop in other parts of coniferous trees, e.g. in rotting top buds of Pinus sibirica Du Tour. Larvae are saprophagous or/and necrophagous, feed on excrements of other inhabitants, dead insects or rotting plant tissues, do not cause primary damage of coniferous cones and seeds (Gaidene \& Nartshuk 1963). Hibernate as larvae or/ and pupae.

\section{Incertella albipalpis (Meigen, 1830)}

Material examined: Murmansk Province. Lps: 2 males, Pechenga; 1 female, Trifonovo; 1 male, 3 females, Lotta River, Poppius, Platonoff (NHMH); Lim: Kirovsk, 1.VIII.1974, Kasparyan (ZISP). Karelia. Kk: 3 males, 1 female, Nikol'skaya Bay, 28.VII.1996, Przhiboro (ZISP); Kon: 1 female, 1 male, Konchezero, 8.VII.1942, Tiensuu (NHMH); 2 males, 2 females, Lisitsyno, 4.VII.2004, Polevoi (FRIP); Kol: 1 male, Gizhino (NHMH).

Distribution: Transpalaearctic polyzonal species, ranges from the British Isles to Japan

Biological notes: Common species of various meadows, forest edges and agricultural fields. Larvae phytophagous or saprophagous, develop in shoots of different grasses including cereals.

\section{Incertella karteshensis Nartshuk \& Przhiboro, 2009}

Material examined: Karelia. Kk: 2 males, 1 female, Kartesh, 3-5. VII.2000, Przhiboro (ZISP).

Distribution: Described and so far only known from Karelia (Nartshuk \& Przhiboro 2009).

Biological notes: Known only from the upper intertidal zone at the sea shore, covered with grassy vegetation (tidal meadow). Collected only with yellow plate traps.

\section{Incertella kerteszi (Becker, 1910)}

Material examined: Murmansk Province. Lim: 1 female, Monchegorsk, 20-24.VII.1995, Kozlov (ZISP). Karelia. Kol: 1 male, Lososinnoe, 12.VII.2012, Polevoi (FRIP); Kl: 1 male, Lake Iso-Iijärvi, 5.VII.2005, Polevoi (FRIP).

Distribution: Transpalaearctic boreal species, distributed from the British Islands to Kamchatka.

Biological notes: Wet meadows and forest edges.

\section{Incertella nigrifrons (Duda, 1933)}

Material examined: Murmansk Province. Lps: 1 male, Lotta River, Platonoff (NHMH). Karelia. Kk: 1 female, Primorskii, 17.VIII.1996, Przhiboro (ZISP); Kon: 1 male, Konchezero, 4-6.VII.2012, Polevoi (FRIP).

Distribution: Eurasian species.

Biological notes: Wetlands. Hygrophilous species. 
*Incertella scotica (Collin, 1946)

Material examined: Karelia. Kk: 1 male, Kartesh, 3.VII.2000, Przhiboro (ZISP).

Published record: Murmansk Province. Lotta River (Nartshuk \& Andersson 2013).

Distribution: European species.

Biological notes: Wetlands. Hygrophilous species.

\section{Incertella zuercheri (Duda, 1933)}

Published record: Karelia. Without locality name (Nartshuk \& Andersson 2013).

Distribution: Eurasian species.

Biological notes: Phragmites beds. Larvae develop as inquilines in galls of Lipara (Diptera, Chloropidae).

\section{Lasiambia coxalis (von Roser, 1840)}

Published record: Karelia. Without locality name (Nartshuk \& Andersson 2013).

Distribution: Eurasian species.

Biological notes: Meadows, Phragmites beds. Larvae carnivorous, feed on eggs of Acrididae (Nartshuk 2015).

\section{Lasiambia palposa (Fallén, 1820)}

Material examined: Karelia. Kpor: 1 female, Nadvoitsy (NHMH); Kon: 1 female, Kondopoga, 22.VIII.2012, A. Kainelainen (ZISP); 2 males, 2 females, Vorob'i, 6-8.VIII.1996, Polevoi (ZISP); 1 male, Solomennoe, Sahlberg (NHMH); Kol: 2 males, 1 female, Gizhino, 15.IX.1941, Tiensuu; 1 male, 2 females, Sheltozero, 25-29.VIII.1942, Tiensuu (NHMH). Leningrad Province. Kol: 3 males, 4 females, Vazhiny, 14.06-28.IX.1942, Tiensuu (NHMH).

Published records: Karelia. Surroundings of Ladoga Lake (Chydenius \& Furuhjelm 1859); Kol: Petrozavodsk (Frey 1934).

Distribution: Widely distributed Palaearctic species, distributed from Europe to Mongolia and Israel.

Biological notes: Meadows and forest edges. Larvae carnivorous, live in and may feed on egg pods of Acrididae. It is possible that larvae will feed on other food too.

\section{Microcercis kroeberi (Duda, 1935)}

Material examined: Karelia. Kk: 5 males, 8 females, Kartesh, 21-25. VII.1973, Sugonyaev, 20.VIII.1996, 3-5.VII.2000, Przhiboro; 1 male, Primorskii, 17.VIII.1996, Przhiboro (ZISP).

Distribution: European species.

Biological notes: Wet meadows and wetlands, including the sea intertidal and supralittoral zone.

\section{Microcercis trigonella (Duda, 1933)}

Material examined: Murmansk Province. Lps: 1 female, Yläluostari (NHMH); 1 female, Pasvik Nature Reserve, Langvatn, 4.VIII.2008, Polevoi (FRIP); 65 females, Lotta River, Poppius (NHMH). Karelia.
Ks: 1 male, Paanajärvi (NHMH); Kk: 3 males, 4 females, Primorskii, 17.VIII.1996, Przhiboro; 2 males, 2 females, +22 specimens, Kartesh, 4.VIII.1996, 3-5.VII.2000, Przhiboro, 26.VII.2010, Nartshuk (ZISP); Kpoc: 1 female, Lake Kuzharvi, 21.VI.2000, Polevoi (FRIP); Kon: 1 female, Konchezero (NHMH); Kl: 1 female, Sortavala (NHMH); Kton: 1 male, Kolgostrov, 10.VIII.1995, Polevoi (ZISP).

Distribution: Eurasian species.

Biological notes: Wet meadows and wetlands including the intertidal and supralittoral zone. Larvae live in stems of Juncus effusus L. and J. gerardii Loisel. (Tschirnhaus 1981).

\section{**Oscinella alopecuri \\ Mesnil in Balachovsky \& Mesnil, 1935}

Material examined: Murmansk Province. Lmur: 1 male, Dal'nie Zelentsy, 6.VIII.1981, Gorodkov (ZISP).

Distribution: Euroasian species.

Biological notes: Meadows. Larvae phytophagous, develop in shoots of Alopecurus spp.

\section{Oscinella cariciphila Collin, 1946}

Material examined: Karelia. Kk: 3 males, 1 female, Kartesh, 16-20. VII.1966, Tanasijtshuk; 21-25.VII.1973, 9-16.VIII.1989, Sugonyaev; 1 pupa (reared to adult), Kartesh, 15.VII.1996, Przhiboro (ZISP); Kon: 2 males, Island Bukol'nikov, 25.VI.2003, Polevoi (FRIP).

Distribution: Eurasian species.

Biological notes: Wet habitats with sedges. Larvae propably live in shoots of Carex spp.

\section{Oscinella frit (Linnaeus, 1758)}

Material examined: Murmansk Province. $L t$ : 1 female, Mishukovo, 7.VIII.1997, Przhiboro (ZISP); Lmur: 1 male, +4 specimens, Dal'nie Zelentsy, 10.VIII.1981, Gorodkov; 4.VIII.1997, Przhiboro (ZISP); Lim: 1 male, 1 female, Monchegorsk, 20-24.VII.1995, Kozlov; 1 female, Island Berezhnoi Vlasov, 16.VIII.1993, Przhiboro (ZISP). Karelia. Kk: 13 males, 9 females, +48 specimens, Kartesh, 16-20.VII.1966, Tanasijtshuk; 23.VII.1975, Gorodkov; 27.VII-16.VIII.1989, Sugonyaev; 23.VII.1975, Gorodkov; 15.VII.1996, 21.VIII.1996, 2.VII.1997, 3-5. VII.2000, Przhiboro; 26.VII.2010, Nartshuk; 3 females, Primorskii, 17.VIII.1996, Przhiboro; 1 male, 2 females, Nikol'skaya Bay, 28.VII.1996, Przhiboro; 12 specimens, Chupa, 30.VI.2000, Przhiboro (ZISP); 1 female, Island Malyi Andronin, 20.VII.2010, Nartshuk (ZISP); 2 females, Syrovatka, 18.VII.2003, Polevoi (FRIP); Kpoc: 1 male, Island Nemetskii Kuzov, 17.VII.2001, Humala; 1 male, Island Lodeinyi, 21.VII.2001, Humala (FRIP); Kpor: 1 female, Segezha, 29.VII.1996, Gorodkov (ZISP); Kon: 2 males, 1 female, Shaidoma, 11.VIII.2018, Polevoi; 1 male, 10 females, Konchezero, 4-6.VII.2012, Polevoi; 1 male, 1 female, Kizhi, 15.VI.1994, Polevoi; 1 male, Island Radkol'e, 30.VII.2018, Polevoi; 1 male, Lelikovo, 24.VI.2003, Polevoi (FRIP); Kol: 5 males, 2 females, Vidlitsa, 3-4.IX.2018, Polevoi; 2 males, Ust'e Tuloksy, 5.IX.2018, Polevoi; Kton: 1 male, Besov Nos, 18.VII.2006, Polevoi (FRIP).

Published records: Karelia. Surroundings of Ladoga Lake (Chydenius \& Furuhjelm 1859); Ks: Paanajärvi (Krogerus 1960); Kton: Besov Nos (Humala \& Polevoi 2009, as O. vindicata Meigen, 1830; misidentification). 
Distribution: One of the commonest species of the family, possibly representing a group of closely related species. Widely distributed species, recorded from all zoogeographical regions of the World.

Biological notes: Meadows, forest edges and agricultural fields. Two or three generations in a year. Larvae phytophagous, develop in shoots and in spikelets of cereals (wheat, barley, rye, oat) and many wild grasses. The species may seriously damage cereals and fodder grasses.

\section{Oscinella maura (Fallén, 1820)}

Material examined: Karelia. Kon: 1 female, Shaidoma, 10.VIII.2018, Polevoi (FRIP).

Published records: Karelia. Surroundings of Ladoga Lake (Chydenius \& Furuhjelm 1859).

Distribution: Known from Europe, Siberia, Caucasus and Iran. Nartshuk \& Andersson (2013) mention Karelia in the "Distribution" section, but not in the catalogue, probably by mistake.

Biological notes: Meadows, forest edges and pastures. Two-three generations in a year. Larvae phytophagous, live in shoots of Dactylis glomerata L.

\section{***Oscinella nigerrima (Macquart, 1935)}

Material examined: Murmansk Province. Lim: 1 female, Kolvitsa, 8.VIII.1995, Gorodkov (ZISP). Karelia. Kpor: 1 male, 1 female, Segezha, 29.VII.1996, Gorodkov (ZISP).

Distribution: Eurasian species.

Biological notes: Meadows. Larvae phytophagous, live in shoots of Poaceae.

\section{Oscinella nitidissima (Meigen, 1838)}

Material examined: Murmansk Province. Lps: 1 male, 2 females, Lotta River, Poppius (NHMH). Karelia. Kk: 1 female, Kartesh, 25.VII.1996, Polevoi (FRIP); 1 male, Kartesh, 21.VIII.1996, Przhiboro (ZISP); Kon: 1 male, Island Bukol'nikov, 30.VII.2018, Polevoi (FRIP); Kl: 1 male, Impilahti (NHMH); 1 female, Kirjavalahti, 4.VII.2005, Polevoi; 1 female, Niva, 7.VII.2005, Polevoi (FRIP); Kol: 2 females, Vidlitsa, 4.IX.2018, Polevoi (FRIP).

Distribution: Holarctic species, widely distributed in the Palaearctic. Biological notes: Meadows, forest edges and agricultural fields. Larvae phytophagous, develop in shoots of Agrostis spp. and probably of other grasses.

\section{Oscinella pusilla (Meigen, 1830)}

Material examined: Karelia. Kk: 22 males, 3 females, Kartesh, 9-20.VII.1966, Tanasijtshuk, 21-25.VII.1983，27.07-16.VIII.1989, Sugonyaev, 6.VI.1993, Przhiboro; 2 pupae (reared to adults), Kartesh, 3.VII.2000, Przhiboro (ZISP); 1 female, Syrovatka, 19.VII.2003, Polevoi (FRIP); Kon: 1 female, Myagrozero, 18.VII.2002, Polevoi; 2 males, 1 female, Vegoruksy, 24-29.VI.2013, Polevoi; 1 female, Kosalma, 15.VII.2002, Polevoi; 1 female, Island Bukol'nikov, 25.VI.2003, Polevoi; 1 female, Pin'guba, 6.VII.1997, Polevoi (FRIP).

Published records: Murmansk Province. Kola Peninsula, without locality name (Nartshuk \& Andersson 2013).

Distribution: Widely distributed in the Palaearctic.
Biological notes: Meadows and agricultural fields. Larvae phytophagous, live in shoots and (more rarely) in spikelets of cereals (usually avoid oats), as well in shoots of different wild grasses, preferably of the tribe Hordeae. A well-known serious pest of cereals in many European countries.

\section{*Oscinella trochanterata Collin, 1946}

Material examined: Karelia. 1 female, Sukhaya Vodla, 24.VIII.2006, Polevoi (FRIP).

Published records: Karelia. Kton: Sukhaya Vodla (Humala \& Polevoi, 2009, as O. vindicata; misidentification).

Distribution: European species.

Biological notes: Wet meadows, edges of ponds and other marshy habitats. Probably, two generations in a year. Larvae phytophagous, develop in unshelled inflorescence of Phalaris arundinacea L. The tissues of the infested plant turn brown above the larvae placement.

\section{*Oscinella ventricosi Nartshuk, 1956}

Material examined: Karelia. Kon: 1 male, Lisitsyno, 4.VII.2004, Polevoi (FRIP).

Distribution: Euroasian species.

Biological notes: Meadows. Larvae phytophagous in shoots of Poaceae.

\section{*Oscinella vindicata (Meigen, 1830)}

Taxonomic notes: Nartshuk \& Andersson (2013) used the junior synonym O. hortensis Collin, 1946 for this species. However, Nartshuk (2006) already formally synonymised these names.

Material examined: Karelia. Kl: 1 male, 1 female, Valaam, 31.VII.2009, Polevoi (FRIP).

Distribution: Euroasian species.

Biological notes: Meadows. Larvae probably phytophagous in stems of Poaceae as other species of Oscinella.

\section{*Oscinimorpha minutissima (Strobl, 1900)}

Material examined: Murmansk Province. Lim: 1 female, Khibiny, 16.VIII.1928, Cheburova (ZISP). Karelia. Kon: 1 female, Island Radkol'e, 30.VII.2018, Polevoi (FRIP).

Distribution: Euroasian species.

Biological notes: Meadows and forest edges. Larvae phytophagous. Plant genera Salvia, Secale and Oryza as well as Melandrium dioicum (L.) Coss. et Germ. were listed as hosts (Tschirnhaus 1981).

\section{*Oscinimorpha sordidissima (Strobl, 1893)}

Material examined: Karelia. Kk: 2 males, 1 female, Kartesh, 3-5. VII.2000, Przhiboro (ZISP).

Distribution: Euroasian species. In Fennoscandia, the species was known only from some southern provinces of Sweden and Finland (Nartshuk \& Andersson 2013). Our record extends its range far north. Biological notes: Wet meadows; our material was collected in the White sea intertidal and supralittoral zone. 


\section{Oscinisoma cognatum (Meigen, 1830)}

Material examined: Karelia. Kk: 1 male, Kartesh, 28.VII.2004, Przhiboro; 1 pupa (reared to adult), Kartesh, 22.VII.2004, Przhiboro (ZISP); Kon: 1 male, Bukol'nikov, 30.VII.2018, Polevoi (FRIP); Kol: 1 male, 1 female, Vidlitsa, 3.IX.2018, Polevoi (FRIP); 1 female, Petrozavodsk, 4.VI.1942, Tiensuu (NHMH). Leningrad Province. Kol: 1 male, Vazhiny, 2.V.1942, Tiensuu (NHMH).

Distribution: Transpalaearctic boreal species.

Biological notes: Common in wetlands. Flies live near the ground in deep litter. Larvae develop in different monocotyledones. Hibernate as adults.

\section{Polyodaspis ruficornis (Macquart, 1835)}

Taxonomic notes: Cherian (2012) considered Polyodaspis Duda, 1933 a junior synonym of Anacamptoneurum Becker, 1903. However, Ebejer \& Andrade (2015) considered this synonymy questionable due to intermediate character states in these and some other related genera. Thus, we prefer to retain the name Polyodaspis until this issue is clarified. Material examined: Murmansk Province. Lps: 1 male, 1 female, Yläluostari, Hellen; Lt: 1 female, Kola; Lim: 2 females, Kandalaksha (all in ZMNH). Karelia. Kon: 1 male, Konchezero, 10.VII.1942, Tiensuu; Kol: 1 female, Petrozavodsk (all in ZMNH).

Distribution: Widely distributed in the Palaearctic and recorded in the Oriental Region as well.

Biological notes: Dry habitats. Larvae feed on different organic matters, usually rich in proteins, and exhibit very wide ecological plasticity in relation to food substrate and enviromental conditions. They may be saprophagous, phytophagous, necrophagous or parasitic (Kiauka \& Nartshuk 1973).

\section{Rhopalopterum anthracinum (Meigen, 1830)}

Material examined: Karelia. Kon: 1 male, Martsyal'nye Vody, Sahlberg (NHMH).

Published records: Murmansk Province. Without locality name (Nartshuk \& Andersson 2013).

Distribution: Eurasian boreal species. Natrshuk \& Andersson (2013) mention Murmansk Province in the catalogue, but not in the "Distribution" section, probably by mistake.

Biological notes: Wetlands with sedges. Species of Carex are probable host plants.

\section{Rhopalopterum atricillum (Zetterstedt, 1838)}

Material examined: Murmansk Province. Lps: 1 male, Lotta River, Poppius (NHMH); Lmur: 1 specimen, Gavrilovo, 21.VII.1913, Frey; 1 specimen, Voron'ya River, 21.VII.1913, Frey (NHMH); Lp: 1 specimen, Ponoi, 18.VII.1913, Frey (NHMH); Lim: 4 males, 10 females, Monchegorsk, 20-24.VII.1995, Kozlov; 2 females, Lake Vudjarv, 15.VII.1931, Fridolin (ZISP). Karelia. Ks: 1 specimen, Paanajärvi, Frey (NHMH).

Distribution: Euroasian boreal species.

Biological notes: Wetlands with sedges. Species of Carex are probable host plants.
Rhopalopterum atricorne (Zetterstedt, 1838)

Material examined: Karelia. 1 female, Karelia, without locality name (NHMH).

Distribution: Eurasian boreal species.

Biological notes: Wetlands with sedges. Species of Carex are probable host plants.

\section{Rhopalopterum brunneipenne \\ Beschovski \& Lansbury, 1987}

Material examined: Karelia. Kk: 2 males, 1 female, Kartesh, 8.VII.1997, Przhiboro (ZISP).

Distribution: European species. Described from England and elsewhere only known from Lithuania and Russian Karelia (Nartshuk \& Przhiboro 2003; Nartshuk \& Andersson 2013).

Biological notes: In northern Karelia, the species was collected from a lake shore.

\section{Rhopalopterum fasciola (Meigen, 1830)}

Material examined: Karelia. Kon: 1 male, Kurgenitsy, 18.VII.2000, Polevoi; Kton: 1 female, Sukhaya Vodla River, 24.VIII.2006, Polevoi (FRIP).

Distribution: Eurasian boreal species.

Biological notes: Wetlands with sedges. Species of Carex are probable host plants.

\section{**Rhopalopterum femorale (Collin, 1946)}

Material examined: Murmansk Province. Lim: 1 female, Monchegorsk, 20-24.VII.1995, Kozlov; 2 females, Lake Vudjarv, 13-30.VII.1931, Fridolin (ZISP). Karelia. Kk: 1 male, 1 female, Syrovatka, 18.VII.2003, Polevoi (FRIP); Kon: 3 females, Island Lyudskoi, 24.VI.2003, Polevoi; 1 female, Lelikovo, 24.VI.2003, Polevoi (FRIP).

Distribution: Eurasian boreal species.

Biological notes: Wetlands with sedges. Species of Carex are probable host plants.

\section{Siphonella oscinina (Fallén, 1820)}

Material examined: Murmansk Province. Lps: 1 female, Pechenga; 1 male, 1 female, Yläluostari (NHMH); Lt: 1 male, Kola (NHMH). Lim: 1 female, Monchegorsk, 20-24.VII.1995, Kozlov; 1 female, Khibiny, 16.VIII.1928, Cheburova (ZISP); 1 female, Kandalaksha (NHMH). Karelia. Kk: 1 female, Kartesh, 22-24.VII.2010, Nartshuk (ZISP); 1 female, Sonostrov, 6.VIII.2006, Polevoi; 1 male, Syrovatka, 19.VII.2003, Polevoi (FRIP); Kpoc: 1 female, Island Nemetskii Kuzov, 22.VIII.2002, Humala; 1 female, Island Zhiloi, 22.VII.2001, Humala (FRIP); Kon: 1 male, Vorob'i, 6-8.VIII.1996, Polevoi (ZISP); 1 female, Vorob'i, 20.VIII.2008, Polevoi (FRIP); 1 female, Gomsel'ga, 3-5. VI.2013, Polevoi; 1 male, Island Bukol'nikov, 30.VII.2018, Polevoi; 1 male, Island Radkol'e, 30.VII.2018, Polevoi (FRIP); Kl: 1 female, Valaam, 30.VII.2009, Polevoi (FRIP); Kol: 1 female, Kolatsel'ga; 2 females, Petrozavodsk (NHMH); 1 female, Vidlitsa, 3.IX.2018, Polevoi; 1 female, Zales'e, 26.VIII.2004, Polevoi (FRIP); 1 male, 
Sändeba (NHMH); Kton: 1 female, Kolgostrov, 10.VIII.1995, Polevoi (ZISP).

Published records: Karelia. Surroundings of Ladoga Lake (Chydenius \& Furuhjelm 1859); Ks: Paanajärvi (Krogerus 1960).

Distribution: Holarctic species.

Biological notes: Dry meadows. The data on bionomics of the species are controversal. Larvae are predators in egg cocoons of Aranei or feed on coccids Greenisca glyceriae (Green) living on Agrostis capillaris L. Both sexes of this species are highly attracted by white pan traps in late summer and autumn which may mirror the attractivity of spider cocoons as a source of oviposition and rendezvous strategy (Tschirnhaus 1992).

\section{Speccafrons halophila (Duda, 1933)}

Material examined: Karelia. Kon: 1 male, 2 females, Konchezero, 7.VII.1942, Tiensuu (NHMH); 1 female, Island Bukol'nikov, 25.VI.2003, Polevoi (FRIP).

Distribution: Transpalaearctic species, distributed from the British Islands to the Far East of Russia.

Biological notes: Meadows and wetlands with Phragmites australis. Larvae carnivorous, develop in egg cocoons of Araneae, which was studied in detail by Rollard $(1987,1992)$.

\section{*Trachysiphonella ruficeps (Macquart, 1835)}

Material examined: Karelia. Kon: 2 males, Gomsel'ga, 4-6.VI.2013, Polevoi (FRIP).

Distribution: Euroasian species.

Biological notes: Dry meadows. The larval substrate is completely unknown.

\section{Trachysiphonella scutellata (von Roser, 1840)}

Material examined: Karelia. Kon: 2 females, Kivach, 4.VII.2001, 3.IX.2002, Polevoi; 1 female, Island Radkol'e, 30.VII.2018, Polevoi; 1 female, Konchezero, 15.VII.2002, Polevoi (FRIP).

Distribution: Eurasian species, occurring from Europe to Mongolia and Israel.

Biological notes: Dry meadows and forest edges.

\section{Tricimba cincta (Meigen, 1830)}

Material examined: Murmansk Province. Lps: 1 male, Lotta River, Poppius; Lt: 2 males, Kola; Lim: 2 males, Kandalaksha (all in ZMNH). Karelia. Kk: 1 female, Kartesh, 21.VIII.1996, Przhiboro; 1 female, Kartesh, 22-24.VII.2010, Nartshuk (ZISP); Kon: 2 females, Semchezero (NHMH); 1 female, Girvas, 16.VII.2002, Polevoi; 1 female, Ar'koila, 20-21.VI.2018, Polevoi; 3 females, Vendery, 29-31. VIII.2017, Polevoi; 1 male, 5 females, Kivach, 26.VI-10.VII.1990, 26.VI-7.VII.2000, 1.VIII.2003, 26.V-23.VI.2016, Polevoi (FRIP); 2 females, Konchezero, 4-6.VII.2012, Polevoi; 1 female, Vorob'i, 20.VIII.2008, Humala (FRIP); 1 female, Yalguba (NHMH); Kl: 1 male, Sortavala (NHMH); Kol: 1 female, Kolatselga; 1 female, Sändeba; 2 females, Petrozavodsk (NHMH); 1 female, Sheltozero, 13.VII.2004, Polevoi (FRIP). Arkhangelsk Province. Kton: 1 female, Vozhosel'ga, 8.VII-2.VIII.2014, Polevoi (FRIP).
Published records: Karelia. Surroundings of Ladoga Lake (Chydenius \& Furuhjelm 1859).

Distribution: Holarctic species.

Biological notes: Meadows, forests and agricultural fields. Recorded within houses in autumn together with Thaumatomyia notata (Meigen, 1830). The species exhibits a wide ecological plasticity in relation to food substrate. It was reared from stems of cereals infested by other insects, from mushrooms, berries of Sambucus racemosa L., dead molluscs, caterpillars. Hibernate as adults.

\section{Subfamily Chloropinae}

\section{Cetema cereris (Fallén, 1820)}

Material examined: Murmansk Province. Lt: 1 male, Murmansk, 19.VIII.1923, Kuznetzov (ZISP); Kk: 1 female, Kovda, 25.VII.1870, Sahlberg (NHMH). Karelia. Ks: 2 specimens, Paanajärvi, 22-27. VII.1934, Ekman; Kk: 1 male, Kartesh, 16-20.VII.1966, Tanasijtshuk (ZISP); Kon: 1 male, Island Bukol'nikov, 30.VII.2018, Polevoi; 1 female, Kivach, 3.IX.2002, Polevoi; 1 female, Kosalma, 15.VII.2002, Polevoi; 1 female, Nizhnee Myagrozero, 20-24.VII.2012, Polevoi; 1 male, 3 females, Pod'el'niki, 18-22.VII.2011, Polevoi (FRIP); 3 males, 1 female, Vorob'i, 6-8.VIII.1996, Polevoi (ZISP), 18-22.VIII.2008, Polevoi, Humala; 1 female, Pin'guba, 30.VI.1991, Polevoi (FRIP); Kol: 1 specimen, Kolatselga, 30.VIII.1943, Tiensuu (NHMH); 1 male, Sudalitsa, 30.VII.1994, Polevoi; 1 male, Kaskesruchei, 20.VII.2004, Polevoi; 1 male, Shoksha, 13.VII.2004, Polevoi (FRIP).

Published records: Murmansk Province. Lt: Murmansk (Nartshuk 1999a). Karelia. Surroundings of Ladoga Lake (Chydenius \& Furuhjelm 1859); Ks: Paanajärvi; Kol: Kolatsel'ga (Nartshuk 1999a).

Distribution: Transpalaearctic boreo-nemoral species, distributed from the Great Britain to Sakhalin and Japan.

Biological notes: Meadows, forest edges and clearings. Larvae phytophagous, develop in shoots of Poaceae.

\section{Cetema elongatum (Meigen, 1830)}

Material examined: Karelia. 1 female, without locality name, Tengström (NHMH); Kk: 1 male, 1 female, Kartesh, 17-22.VII.2010, Nartshuk (ZISP); Kl: 1 male, Salmi, 11.VIII.1941, Tiensuu; 1 male, 1 female, Sortavala, Tiensuu, Woldstedt; 1 female, Valaam, Suomalainen; Kol: 1 female, Derevyannoe, 2.VII.1942, Tiensuu; 1 female, Vedlozero, 18.VII.1942, Tiensuu; 1 female, Vidlitsa, 23.VII.1942, Tiensuu (all in ZMNH); Kon: 1 male, Island Bukol'nikov, 30.VII.2018, Polevoi (FRIP).

Distribution: European species.

Biological notes: Meadows, forest edges. Larvae phytophagous, develop in shoots of Poaceae. Univoltine species, overwinters as larvae.

\section{Cetema myopinum (Loew, 1866)}

Material examined: Karelia. Kk: 1 male, Syrovatka, 20-22.VII.2003, Polevoi (FRIP); Kpoc: 1 male, Kostomuksha, 31.VII.1996, Gorodkov (ZISP); Kpor: 1 male, Segezha, 28.VII.1996, Gorodkov (ZISP); Kl: 1 male, Puikkola, 27.VII-21.VIII.1991, Polevoi; 1 male, Haapalampi, 2.VII.2010, Polevoi; 1 male, Reuskula, 6.VII.2010, Polevoi; 1 male, Sukopohja, 7.VII.2005, Polevoi; 3 males, 1 female, Niva, 7.VII.2005, 
Polevoi; 1 male, Kurkijoki, 6.VII.2005, Polevoi; (FRIP); 1 male, Valaam, Suomalainen (NHMH); Kon: 1 male, Lake Nizhnii Myarat, 6.VII.2006, Polevoi; 1 male, Tipinitsy, 4.VII.2004, Polevoi; 1 male, Konchezero, 4.VII.2012, Polevoi; 1 female, Vorob’i, 27-28.VII.1994; 1 male, 1 female, Oyatevschina, 21.VII.2011, Polevoi; 1 male, Vegoruksy, 25-28.VI.2013, Polevoi (FRIP); Kol: 1 male, Derevyannoe, 2.VII.1942, Tiensuu (NHMH).

Published records: Karelia. Without locality name (Nartshuk \& Andersson 2013).

Distribution: Eurasian species, distributed from the Great Britain to Lake Baikal and northern Mongolia.

Biological notes: Meadows. Larvae phytophagous, develop in shoots of different grasses, but prefer species of the genus Agrostis.

\section{Cetema simile Ismay, 1985}

Taxonomic notes: There are different opinions on the identity of $C$. simile. Some authors treat it as a junior synonym of $C$. elongatum (Savage \& Wheeler 1999; Nartshuk \& Andersson 2013), others consider it as a distinct species (Merz 2012; Nartshuk \& Kahanpää 2014). Here we follow the latter authors, accepting that these species are reliably differentiated at least in the shape of hypandrium (see Ismay 1985; Nartshuk 1999b).

Material examined: Karelia. Kl: 1 male, Valaam, 30.VII.2009, Polevoi (FRIP).

Published records: Karelia. Kl: Sortavala, Vidlitsa (Nartshuk 1999a).

Distribution: European species.

Biological notes: Meadows. Larvae probably phytophagous as other species of Cetema.

\section{Chlorops anthracophagoides Strobl, 1901}

Material examined: Karelia. Kl: 1 female, Impilahti, Suomalainen; Kol: 1 male, Gizhino, Platonoff (NHMH); 1 male, Kolatsel'ga, 18.VI.1942, Tiensuu (all in ZMNH).

Distribution: Euro-Caucasian species.

Biological notes: Meadows. Larvae probably phytophagous as other species of Chlorops.

\section{**Chlorops calceatus Meigen, 1830}

Material examined: Murmansk Province. Lps: 1 female, Korablekk Mountain, 30.VII.2008, Polevoi (FRIP); Lm: 1 female, Lovozero, 16.VIII.1981, Gorodkov (ZISP). Karelia. Kon: 1 male, Lizhma, 3.VIII.1936, Cheburova (ZISP); Kl: 1 female, Impilahti, Suomalainen (NHMH); Kol: 1 male, Derevyannoe, 2.VII.1942, Tiensuu (NHMH). Distribution: Euroasian species.

Biological notes: Meadows and forest edges. Larvae phytophagous, host plant Festuca rubra L. (Tschirnhaus 1981).

\section{*Chlorops centromaculatus (Duda, 1933)}

Material examined: Karelia. Kon: 1 male, 1 female, Island Sychevets, 1.VIII.2018, Polevoi (FRIP).

Distribution: Rare European species.

Biological notes: Meadows. Autumnal species.

\section{Chlorops figuratus (Zetterstedt, 1848)}

Material examined: Karelia. Ks: 1 male, Paanajärvi, Frey (NHMH). Distribution: Eurasian species.

Biological notes: Meadows. Larvae probably phytophagous as other species of Chlorops.

\section{Chlorops frontosus Meigen, 1830}

Material examined: Karelia. Kon: 1 male, Lelikovo, 24.VI.2003, Polevoi (FRIP).

Distribution: European species.

Biological notes: Fens and marshes with sedges. Larvae phytophagous, develop in shoots of large species of Carex (Cyperaceae).

\section{Chlorops geminatus Meigen, 1830}

Material examined: Karelia. Kk: 1 female, Syrovatka, 16.VII.2003, Polevoi (FRIP); Kl: 1 male, Ruskeala, 28.VII.1949, Nyland (NHMH). Distribution: Eurasian species.

Biological notes: Meadows. The species was reared from Hierochloe odorata (L.) P. Beauv. (Karpova 1972).

\section{Chlorops gracilis Meigen, 1830}

Material examined: Karelia. Kon: 1 male, Tivdiya, 22.VI.1943, Hellen (NHMH); 1 male, Konchezero, 4.VII.2012, Polevoi (FRIP); Kol: 1 male, Shoksha, 13.VII.2004, Polevoi; 1 male, Mayachino, 22.VI.2012, Humala (FRIP).

Distribution: Eurasian species.

Biological notes: Dry meadows. One generation in a year. Hibernate as larvae in shoots of Calamagrostis epigejos (L.) Roth.

\section{Chlorops hypostigma Meigen, 1830}

Material examined: Karelia. Kpoc: 1 female, Island Taparukha, 17.VIII.2002, Humala (FRIP); Kon: 1 male, Oyatevschina, 21.VII.2011, Polevoi (FRIP); Kl: 1 female, Ruskeala, 28.VII.1949, Nyland (NHMH); Kol: 1 female, Kolatsel'ga, 2.VIII.1942, Tiensuu; 2 males, 1 female, Olonets, 25.VII.1942, Tiensuu (NHMH); 1 female, Sheltozero, 24.VIII.2004, Polevoi; 1 male, 1 female, Kaskesruchei, 19-20.VII.2004, Polevoi (FRIP).

Distribution: Euro-Caucasian species.

Biological notes: Meadows and forest edges. Univoltine species, overwinters as the third instar larvae in shoots of Poaceae.

\section{Chlorops kirigaminensis Kanmiya, 1978}

Material examined: Karelia. Ks: 1 male, Paanajärvi (NHMH). Published records: Karelia. Ks: Paanajärvi (Nartshuk 1998, as C. zonulatus auct. non Wahlgren, 1913).

Distribution: Widely distributed in the Palaearctic Region eastwards to Japan. More common in the Asian part of the range.

Biological notes: Meadows. Larvae probably phytophagous as other species of Chlorops. 


\section{Chlorops laetus Meigen, 1830}

Material examined: Karelia. Kol: 1 female, Sheltozero, 3.IX.1942, Tiensuu (NHMH); 1 female, Matveeva Sel'ga, 26.VIII.2004, Polevoi (ZISP).

Distribution: The species is known from Europe and Kazakhstan. Biological notes: Meadows. Autumnal species.

\section{Chlorops limbatus Meigen, 1830}

Material examined: Karelia. Kk: 1 male, 2 females, Kartesh, 26.VII.2010, Nartshuk; 1 female, Kuzema, 13.V.1935, Cheburova (ZISP); Kpor: 2 males, Valdai, 29.VI.2010, Polevoi (FRIP); Kon: 1 female, Lake Nizhnii Myarat, 6.VII.2006, Polevoi; 1 female, Lipovitsy, 25.VI.2013, Polevoi; 2 males, Polya, 26.VI.2013, Polevoi; 1 male, Tipinitsy, 4.VII.2004, Polevoi; 1 female, Gomsel'ga, 5.VII.2012, Polevoi; 1 female, Kurgenitsy, 19.VII.2000, Polevoi; 1 female, Island Bukol'nikov, 25.VI.2003, Polevoi; 1 female, Island Ernitskii, 24-27. VI.2003, Polevoi; 3 males, 1 female, Klimenitsy, 12.VII.1997, Polevoi (FRIP); Kl: 1 female, Sortavala, Suomalainen; 1 male, 1 female, Valaam, Suomalainen (NHMH), 31.VII.2009, Polevoi (FRIP); 2 females, Island Kilpola, 12-16.VI.2011, Polevoi (FRIP); Kol: 1 female, Ust'e Obzhanki, 23.VI.2012, Polevoi; 3 females, Mayachino, 21-22.VI.2012, Polevoi; 1 male, Sheltozero, 13.VII.2004, Polevoi; 5 males, 2 females, Vidlitsa, 3-4.IX.2018, Polevoi; 5 females, Ust'e Tuloksy, 5.IX.2018, Polevoi (FRIP); Kon: 1 female, Island Bukol'nikov, 30.VII.2018, Polevoi; 1 male, Shaidoma, 11.VIII.2018, Polevoi (FRIP); Kton: 1 male, Vodlozerskii National Park, Novguda River, 20.VIII.2007, Polevoi (FRIP). Kp: 2 females, Ust'-Reka, 25.VI.2009, Polevoi (FRIP).

Published records: Murmansk Province. Ks: Kuolajärvi (Nartshuk 1998). Karelia. Kk: Kuzema (Nartshuk 1998); Kl: Sortavala (Frey 1934, as Oscinis brevimana Loew, 1866); Salmi (Nartshuk 1998).

Distribution: Transpalaearctic species, distributed from the British Isles to Japan.

Biological notes: Wet meadows at river banks and lake shores. Two generations in a year. Larvae phytophagous, develop in shoots of Phalaroides arundinacea (L.) Rauschert; larvae of the summer generation develop in exposed panicles. Tissues of leaves above the larva turn brown, and central leaf dries out. Larvae hibernate in basal and aerial shoots and pupate next spring.

\section{Chlorops meigenii Loew, 1866}

Material examined: Murmansk Province. Lps: 1 female, Borisoglebskii, Hellen; 1 male, 1 female, Kuvernöörikoski, Hellen; 1 female, Zemlyanoe, Frey; 1 male, 2 females, Salmijärvi, Frey, Hellen; 7 males, Trifonovo, 11.VII.1929, Hellen, Lindberg; 5 males, 2 females, Yläluostari, 8.VII.1929, Lindberg (NHMH); Lt: 1 male, Kola, Frey (NHMH); 1 female, Murmansk, 18.VII.1923, Kapustin (ZISP); Lmur: 1 male, 1 female, Lake Seidozero, Palmen (NHMH); Lim: 10 females, Monchegorsk, 20-24.VII.1995, Kozlov (ZISP); 1 male, 2 females, Laplandskii Nature Reserve, Lisii Stream, 25.VII.2013, Polevoi (FRIP); 1 female, Apatity, 7.VII.1998, Anufriev, Mokrousov (ZISP); 2 males, Kandalaksha, Hellen (NHMH); $L p$ : 1 female, Ponoi, Frey (NHMH). Karelia. Ks: 2 males, 2 females, Leppyalä, 1.VII.2000, Polevoi (FRIP); 1 male, 1 female, Paanajärvi, Frey (NHMH); Kk: 1 male, 6 females, Kartesh, 18-22.VII.1975, Gorodkov (ZISP), 24-25.VII.1996, Polevoi (FRIP); 3 females, Keret', 13.VIII.1966,
Tanasijtshuk (ZISP); 1 female, Gridino, 2.VIII.2007, Polevoi (FRIP); 5 males, 3 females, Kyatka River, 5.VII-4.VIII.2007, Polevoi; 30 males, 8 females, Syrovatka, 16-22.VII.2003, Polevoi (FRIP); Kрос: 1 female, Kostomuksha, 1.VI.1996, Gorodkov; 2 females, Kostomukshskii Nature Reserve, 1.VIII.1996, Gorodkov (ZISP); 1 female, Ladvozero, 9.VII.1996, Polevoi (ZISP); 1 male, 2 females, Island Lodeinyi, 21.VII.2001, Humala; 1 female, Island Nemetskii Kuzov, 17.VII.2001, Humala; 1 male, Murdojoki River, 6.VII.2009, Polevoi; 3 females, Nesterova Mountain, 23.VI.2000, Polevoi (FRIP); Kpor: 1 female, Island Bol'shoi Zhuzhmui, 23.VII.2001, Humala; 1 male, 6 females, Island Malyii Zhuzhmui, 25.VII.2001, Humala; 1 female, Island Myagostrov, 14.VIII.2002, Polevoi; 1 female, Island Pechak, 24.VII.2001, Humala (FRIP); 1 female, Sumskii Posad, 24.VII.1996, Gorodkov (ZISP); 2 males, Ladozero, 29.VI.2010, Polevoi; 2 males, 1 female, Valdai, 29.VI.2010, Polevoi (FRIP); Kon: 1 male, Medvezh'egorsk, Carpelan (NHMH); 1 female, Ar'koila, 20.VI.2018, Polevoi; 1 female, Raiguba, 11.VII.2002, Polevoi; 2 males, Lake Vikshezero, 28.VI.2007, Polevoi; 4 males, 9 females, Tereki, 21.VII.2017, 25.VI.2018, Polevoi; 2 males, 2 females, Island Paleostrov, 3.VII.2004, Polevoi; 2 males, Polya, 26.VI.2013, Polevoi; 2 males, Kivach, 31.VII-13.VIII.1990, Polevoi; 1 female, Gomsel'ga, 5.VII.2012, Polevoi (FRIP); 3 females, Konchezero, 10.VII.1942, Tiensuu (NHMH); 1 female, Island Eglov, 26.VI.2014, Polevoi; 2 females, Island Ernitskii, 26.VI.2003, Polevoi; 1 male, 2 females, Island Khvost, 27.VI.2014, Polevoi; 2 males, Island Kuivakhda, 24.VI.2003, Polevoi; 1 female, Island Lyudskoi, 24.VI.2003, Polevoi; 1 female, Island Myal', 2.VII.2017, Polevoi; 1 female, Klimenitsy, 12.VII.1997, Polevoi (FRIP); Kl: 1 female, Ruskeala, Sahlberg; 1 male, Sortavala, Suomalainen; 1 male, Harlu, Tiensuu; 4 males, Impilahti, Forsius, Suomalainen (NHMH); 2 males, Lake Iso-Iijärvi, 5.VII.2005, Polevoi; 1 male, Meijeri, 3.VII.2010, Humala; 1 male, 1 female, Niva, 7-8.VII.2005, Polevoi (FRIP); 2 females, Salmi, 2-3. VI.1910, Tuomikoski, 2.VIII.1941, Tiensuu (NHMH); Kol: 1 female, Kindasovo, 9.VII-5.IX.1996, Polevoi (ZISP); 2 males, Lososinnoe, 9.VII.2012, Polevoi (FRIP); 1 female, Petrozavodsk, 7.VIII.1923, Fridolin; 2 females, Sheltozero, 13.VII.2004, Polevoi; 3 males, 5 females, Vidlitsa, 4.IX.2018, Polevoi; 1 male, Mayachino, 22.VI.2012, Polevoi; 4 males, 1 female, Novikovo, 2.VII.2008, Polevoi (FRIP); 1 male, Gizhino, Platonoff (NHMH); 1 male, Karelia, Tengstrom (NHMH).

Published records: Murmansk Province. Ks: Kuolajärvi; Lmur: Voroninsk (Nartshuk 1998). Karelia. Kon: Velikaya Niva (Nartshuk 1998).

Distribution: Transpalaearctic species, distributed eastwards to Kamchatka.

Biological notes: Wet meadows, wet clearings in the forests. Host plants are probably some species of Calamagrostis other than $C$. epigejos.

\section{Chlorops nigripalpis (Duda, 1933)}

Material examined: Karelia. 1 female, without locality name, Tengström (NHMH).

Distribution: The species is known from Europe and Kazakhstan.

Biological notes: Bionomics unknown. 


\section{Chlorops planifrons Loew, 1866}

Material examined: Murmansk Province. Lps: 1 female, Lotta River, Poppius; 1 female, Pechenga, Frey; 1 female, Salmijärvi, 28.VI.1937, Nordman; 2 males, 1 female, Yläluostari, Frey (NHMH); Lt: 1 male, 1 female, Kola, Frey (NHMH); 1 female, Kola, 4.VII.1906, Soldatov (ZISP); Lm: 1 female, Lovozero, 16.VIII.1981, Gorodkov (ZISP). Karelia. Kpoc: 1 female, Kem', Sahlberg (NHMH); Kpor: 1 female, Segezha, 20.VII.1996, Gorodkov (ZISP); Kon: 1 male, Tipinitsy, 4.VII.2004, Polevoi; 1 female, Island Khvost, 27.VI.2014, Polevoi (FRIP); Kl: 1 female, Valaam, 28.VII.2009, Polevoi (FRIP); Kton: 3 females, Sheltoporog, 2.VIII.2002, Polevoi; Kton: 1 female, Vozritsy, 1.VIII.2002, Polevoi (FRIP).

Distribution: Widely distributed transpalaearctic polyzonal species, distributed from the British Islands to the Far East of Russia, in Europe occurs southwards to Spain.

Biological notes: Fens and marshes with large species of sedges. One generation in a year. Larvae phytophagous, develop in shoots of Carex rostrata Stokes. Larvae of the first instar hibernate in shoots of host plant and continue feeding in the next spring, make a furrow on stem, starting below inflorescence and going down gradually widening until suddenly interrupts. Interruption most likely corresponds to the period of larvae moulting, as furrow becomes wider after interruption. Larvae pupate within stem below. Some larvae leave the stem and continue feeding in another one (Nartshuk 1962).

\section{Chlorops pumilionis (Bjerkander, 1778)}

Material examined: Karelia. Kpor: 1 male, island Nemetskii Kuzov, 17.VII.2001, Humala; 1 female, Island Myagostrov, 14.VIII.2002, Humala (FRIP). Leningrad Province. Kol: 1 male, Vazhiny, 9.IX.1942, Tiensuu (NHMH); 1 male, Gimreka, 2.VI.2007, Polevoi (FRIP).

Published records: Karelia. Ks: Paanajärvi (Krogerus 1960); Kl: Salmi, Sortavala, Yakkima; Kol: Gizhino, Olonets, Petrozavodsk, Sheltozero, Sändeba (Nartshuk 1998).

Distribution: Eurasian temperate species, distributed from Europe to Iran, Israel, West Siberia and Mongolia.

Biological notes: Meadows and agricultural fields. Two generation in a year. Well-known pest of cereals (predominantly wheat, more rarely, barley and rye), but does not develop on oats. Larvae of winter generation develop in shoots of cereals causing thickening of the attacked shoots ("gout swelling"), while larvae of summer generation feed on exposed ear, lower spikeletts and stem below the spike, gnawing a furrow. Different authors also list many species of wild grasses as possible host plants, but most likely only Elymus repens and some other species of closely related genera are genuine ones.

\section{Chlorops ringens Loew, 1866}

Material examined: Karelia. Kon: 1 male, Polya, 26.VI.2013, Polevoi; 1 male, Vorob'i, 19.VIII.2008, Polevoi (FRIP); Kol: 1 female, Ust'e Tuloksy, 5.IX.2018, Polevoi; 2 females, Vidlitsa, 4.IX.2018, Polevoi; 2 females, Matveeva Sel'ga, 26.VIII.2004, Polevoi; 1 female, Zales'e, 26.VIII.2004, Polevoi (FRIP).

Distribution: Eurasian temperate species, distributed from southern Sweden and Finland southwards to Bulgaria and eastwards to Yakutia. Biological notes: Meadows. Autumnal species.

\section{*Chlorops rossicus Smirnov, 1955}

Material examined: Murmansk Province. Lps: 1 male, Salmijärvi, 28.VI.1937, Nordman; 1 male, Yläluostari, 8.VII.1929, Lindberg (NHMH). Lmur: 1 male, Voron'ya River, Hayren (NHMH). Karelia. Kon: 1 female, Vottovaara Mountain, 18.VII.2008, Polevoi (ZISP); 1 female, Island Ernitskii, 26.VI.2003, Polevoi; 1 female, Polya, 26.VI.2013, Polevoi (FRIP).

Distribution: East European species. Repeatedly collected in Germany (Tschirnhaus 1992).

Biological notes: Meadows, forest edges and clearings.

Chlorops rufinus (Zetterstedt, 1848)

Material examined: Karelia. Ks: 1 female, Paanajärvi, Hellen (NHMH).

Distribution: Widespread transpalaearctic species, distributed southwards to Bulgaria and eastwards to Mongolia and Japan, but everywhere rare.

Biological notes: Meadows.

\section{Chlorops scalaris Meigen, 1830}

Material examined: Karelia. Kpoc: 7 males, 2 females, Ladvozero, 11.VII.1996, Polevoi (ZISP); 1 male, Nesterova Mountain, 23.VI.2000, Polevoi (FRIP); Kon: 2 females, Padany, 17.VI.1921, Olonets Expedition (ZISP); 1 female, Lisitsyno, 4.VII.2004, Polevoi (FRIP); 1 female, Konchezero, 10.VII.1942, Tiensuu (NHMH); 2 females, Island Bukol'nikov, 25.VI.2003, Polevoi (FRIP); 1 female, Yalguba, Sahlberg (NHMH); Kl: 1 female, Sortavala, Suomalainen (NHMH); 1 female, Haapalampi, 2.VII.2010, Polevoi (FRIP); Kol: 2 males, 1 female, Kolatsel'ga, 29.VI.1942, Tiensuu (NHMH); Kp: 1 female, Korbozero, 24.VI.1996, Polevoi (FRIP).

Published records: Karelia. Surroundings of Ladoga Lake (Chydenius \& Furuhjelm 1859); Kl: Impilahti, Valaam (Nartshuk 1998); Kрос: Voknavolok (Nartshuk 1998).

Distribution: Eurasian polyzonal species, distributed southwards to Italy and Bulgaria, and eastwards to East Siberia and Mongolia.

Biological notes: Rather common on meadows. Larvae phytophagous, host plants Holcus lanatus L. and Poa pratensis L. (Tschirnhaus 1981).

\section{Chlorops scutellaris (Zetterstedt, 1838)}

Material examined: Murmansk Province. Lt: 1 male, Kola, Frey (NHMH). Ks: 1 specimen, Kuolajärvi, 23.VII.1934, Krogerus (NHMH). Lim: 1 female, Lake Bolshoi Vudjavr, 21.VII.1934, Fridolin; 15 females, Khibiny, 15.VIII.1928, Cheburova, 14.VII.1934, Fridolin (ZISP). Karelia. Kk: 1 female, Kartesh, 17.VII.2010, Nartshuk (ZISP). Published records: Murmansk Province. Lt: Kola (Frey 1934, as Oscinis freyi Duda, 1933). Karelia. Ks: Paanajärvi (Krogerus 1960, as $O$. freyi). This record most probably corresponds to the specimen from Kuolajärvi listed in the examined material.

Distribution: North Eurasian species, distributed from North Europe eastwards to Yakutia.

Biological notes: Wet meadows and fens. 


\section{*Chlorops serenus Loew, 1866}

Karelia: Kol: 1 female, Ust'e Tuloksy, 5.IX.2018, Polevoi (FRIP). Distribution: Transpalaearctic species, known from Europe, Israel and Algeria. Also reported from Morocco (Ebejer \& Kettani 2016), Turkey (Kubík \& Barták 2014), Iran (Khaghaninia \& Gharajedaghi 2013) and Korea (Ryu 1994).

Biological notes: Meadows. Probably, two generations.

\section{Chlorops speciosus Meigen, 1830}

Material examined: Murmansk Province. Lps: 1 male, 1 female, Zemlyanoe, Frey; 1 female, Pechenga, Hellen; 2 males, 3 females, Salmijärvi, 28.VI.1937, Nordman; 5 males, 5 females, Yläluostari, Frey, Hellen (NHMH); 1 female, Pasvik Nature Reserve, Island Niilansaari, 29.VII.2008, Polevoi (ZISP); Lt: 2 females, Polyarnyi, 10.VIII.1923, Kuznetzov; 2 females, Murmansk, 22.VIII.1923, Fridolin (ZISP); 1 male, Kola, Frey (NHMH); Lim: 5 females, Monchegorsk, 20-24. VII.1995, Kozlov; 1 female, Apatity, 7.VII.1998, Anufriev, Mokrousov (ZISP); 1 male, Kandalaksha, Sahlberg (NHMH); Lv: 1 female, Varzuga, 16.VIII.1995, Gorodkov (ZISP). Karelia. Ks: 5 males, 5 females, Paanajärvi, Frey (NHMH); 2 males, Nuorunen Mountain, 12.VII.1990, Jakovlev (FRIP); Kk: 5 males, 3 females, Kartesh, 16-20.VII.1966, Tanasijtshuk, 27.VII.1989, Sugonyaev (ZISP), 22-25.VII.1996, Polevoi (FRIP); 9 males, 5 females, Gridino, 9.VIII.2006, 2-13.VII.2007, Polevoi; 1 female, Kyatka River, 4.VIII.2007, Polevoi; 2 males, Syrovatka, 16-18.VII.2003, Polevoi (FRIP); Kpoc: 8 males, 2 females, Ladvozero, 11.VII.1996, Polevoi (ZISP); 2 females, Voknavolok, Hellen (NHMH); 8 females, Nesterova Mountain, 23.VI.2000, Polevoi (FRIP); Kpor: 2 males, 2 females, Valdai, 29.VI.2010, Polevoi (FRIP); Kon: 1 female, Lake Nizhnii Myarat, 6.VII.2006, Polevoi (FRIP); 3 females, Padany, 17.VI.1921, Olonets Expedition (ZISP); 1 male, Shaidoma, 10.VIII.2018, Polevoi; 1 female, Girvas, 16.VII.2002, Polevoi; 1 male, 1 female, Ar'koila, 19-20.VI.2018, Polevoi; 1 male, 1 female, Vikshezero, 28.VI.2007, Polevoi; 1 female, Nizhnee Myagrozero, 20.VII.2012, Polevoi; 1 male, 1 female, Kivach, 29.VI.1993, 4.VII.2001, Polevoi; 1 female, Gomsel'ga, 5.VII.2012, Polevoi (FRIP); 1 male, Konchezero, 10.VII.1942, Tiensuu (NHMH); 1 female, Pod'el'niki, 21.VII.2011, Polevoi; 1 male, Island Bol'shoi Klimenetskii, 7.VIII.1996, Polevoi; 1 female, Island Bukol'nikov, 30.VII.2018, Polevoi; 1 female, Island Sychevets, 1.VIII.2018, Polevoi; 1 male, 1 female, Lelikovo, 28.VII.2018, Polevoi; 1 male, Klimenitsy, 11.VII.1997, Polevoi; 2 females, Island Eglov, 26-29.VI.2014, Polevoi; 1 male, Gomsel'ga, 9.VIII.2018, Polevoi; 2 females, Pin'guba, 29.VI.1997, 16.VII.2000, Polevoi (FRIP); Kl: 2 males, 1 female, Sortavala, Tiensuu; 1 female, Kirjavalahti, Poppius; 1 female, Harlu, Tiensuu; 1 male, 1 female, Impilahti, Suomalainen, Woldstedt (NHMH); 2 females, Meijeri, 3.VII.2010, 11.VI.2015, Polevoi (FRIP); 1 male, 1 female, Valaam, Suomalainen (NHMH), 31.VII.2009, Polevoi (FRIP); 1 female, Jakkima, Forsius (NHMH); Kol: 1 female, Kolatsel'ga, 3.VIII.1942, Tiensuu; 1 female, Vedlozero, 18.VII.1942, Tiensuu; 1 male, 2 females, Vidlitsa, 22.VII.1942, Tiensuu (NHMH), 4.IX.2018, Polevoi (FRIP); 2 females, Olonets, 27.VII.1942, Tiensuu; 1 male, 2 females, Petrozavodsk, 1.VI24.VIII.1942, Tiensuu (NHMH), 25.VII.2015, Humala (FRIP); 2 males, 3 females, Sheltozero, 26-31.VIII.1942, Tiensuu (NHMH); Kton: 1 female, Sheltoporog, 2.VIII.2002, Polevoi; Kton: 2 females, Vozritsy, 1.VIII.2002, Polevoi (FRIP); Kp: 2 females, Korbozero, 22-24.VI.1996, Polevoi (FRIP). Leningrad Province. Kol: 1 male, Lahta, 10.VII.1992, Polevoi; 1 male, Moshnich'e, 3.VII.2008, Polevoi (FRIP); 1 female, Vazhiny, 20.VII.1942, Tiensuu (NHMH).
Published records: Murmansk Province. Lps: Vuoremijärvi; Lt: Notozero, Tuloma River; Lp: Ponoi (Nartshuk 1998). Karelia. Surroundings of Ladoga Lake (Chydenius \& Furuhjelm 1859); Kk: Chupa, Keret' (Nartshuk 1998).

Distribution: Eurasian species, southwards to Spain and Bulgaria, eastwards to West Siberia (Altai).

Biological notes. Wet meadows. Larvae phytophagous, live in stems of Deschampsia cespitosa (L.) P. Beauv. Hibernate as larvae in the basal tillers.

\section{Chlorops troglodytes (Zetterstedt, 1848)}

Material examined: Murmansk Province. Lim: 1 female, Khibiny, 23.VIII.1928, Cheburova (ZISP); 1 female, Kokorin Stream, 26.VII.2013, Polevoi (FRIP); 1 female, Apatity, 17.VII.1998, Anufriev; 8 females, Umba, 27.VII.1966, Tanasijtshuk (ZISP). Karelia. Ks: 2 males, 2 females, Paanajärvi, 22-27.VII.1934, Ehman (NHMH); Kk: 10 males, 11 females, Kartesh, 16-20.VII.1966, Tanasijtshuk, 6.VIII.1982, Zaitzev, 27.VII-16.VIII.1989, Sugonyaev (ZISP), 28.VII.1996, Polevoi (FRIP); 2 males, 5 females, Gridino, 5-9. VIII.2006 (ZISP), Polevoi, 7.VIII.2007, Humala (FRIP); Kpoc: 1 male, Island Lodeinyi, 21.VII.2001, Humala (ZISP); Kpor: 3 females, Island Bol'shoi Zhuzhmui, 23.VII.2001, Humala (ZISP); 1 female, Island Malyii Zhuzhmui, 25.VII.2001, Humala; 4 males, 3 females, Island Pechak, 24.VII.2001, Humala (FRIP); Kon: 1 male, Vottovaara Mountain, 18.VII.2008, Polevoi; 1 male, Kivach, 29.VI.1993, Polevoi; 1 male, 3 females, Gomsel'ga, 9.VIII.2018, Polevoi; 1 male, Kosalma, 15.VII.2002, Polevoi; 1 female, Pod'elniki, 20.VII.2011, Polevoi; 2 males, Vorob'i, 7.VIII.1996, Polevoi (ZISP), 3 males, 1 female, Vorob'i, 19.VIII.2008, Polevoi; 1 female, Island Sychevets, 1.VIII.2018, Polevoi; 3 males, 1 female, Island Radkol'e, 30.VII.2018, Polevoi; 2 males, 2 females, Island Karel'skii, 31.VII.2018, Polevoi (FRIP); Kl: 1 male, 3 females, Valaam, 27-31.VII.2009, Polevoi (FRIP); Kol: 1 male, Sheltozero, 28.VII.1942, Tiensuu (NHMH).

Published records: Murmansk Province. Ks: Kuolajärvi (Nartshuk 1998). Karelia. Kk: Chupa (Nartshuk 1998).

Distribution: Eurasian polyzonal species, distributed from the Great Britain to East Siberia and Mongolia.

Biological notes: Meadows.

\section{Chlorops varsoviensis Becker, 1910}

Material examined: Karelia. Kk: 1 male, Kartesh, 21-25.VII.1973, Sugonyaev (ZISP); Kl: 1 male, Ruskeala, Sahlberg; 2 females, Salmi, Tuomikoski (NHMH); Kol: 1 male, Lososinnoe, 9.VII.2012, Polevoi (FRIP).

Published records: Murmansk Province. Ks: Kuolajärvi (Nartshuk 1998).

Distribution: Transpalaearctic species, distributed from France to Mongolia and Japan, southwards to Spain, northern Italy and Bulgaria. Biological notes: Wet habitats with sedges. Larvae phytophagous, develop in stems of Carex spp.

\section{Cryptonevra flavitarsis (Meigen, 1830)}

Published records: Karelia. Without locality name (Nartshuk \& Andersson 2013).

Distribution: Transpalaearctic species. 
Biological notes: Phragmites beds. Larvae usually develop as inquilines in galls of Lipara spp., probably also in damaged stems of Phragmites.

\section{Diplotoxa messoria (Fallén, 1820)}

Material examined: Karelia. Kk: 1 male, Kartesh, 4.IX.2005, Przhiboro (ZISP); Kton: 3 males, Vozritsy, 1.VIII.2002, Polevoi (FRIP).

Published records: Karelia. Surroundings of Ladoga Lake (Chydenius \& Furuhjelm 1859); Kl: Sortavala, Jaakkima (Nartshuk 1999a).

Distribution: Holarctic species, widely distributed in the Palaearctic from the Great Britain to the Far East of Russia.

Biological notes: Wet habitats with spike sedges. Larvae develop in Eleocharis spp.

\section{Diplotoxa dalmatina Strobl, 1900}

Material examined: Karelia. Kton: 1 female, Vozritsy, 1.VIII.2002, Polevoi (FRIP).

Published records: Karelia. Kol: Bol'shie Gory (Nartshuk 1999a).

Distribution: Transpalaearctic species, distributed from Europe to the Far East of Russia.

Biological notes: Wetlands.

\section{Epichlorops puncticollis (Zetterstedt, 1848)}

Material examined: Karelia. Kl: 1 female, Suistamo, Tuomikoski (NHMH).

Distribution: Holarctic species.

Biological notes: Wet meadows and marshes with sedges. Larvae develop in Carex spp.

\section{*Lasiosina albipila (Loew, 1866)}

Material examined: Karelia. Kol: 1 male, Vidlitsa, 4.IX.2018, Polevoi (FRIP).

Distribution: European species with a record from Turkey (Kubík \& Barták 2014)

Biological notes: Meadows. It was found in Switzerland that adults feed on secreta of Agelastica alni (Linnaeus, 1758) larvae (PschornWalcher 1956).

\section{Lasiosina herpini (Guérin-Ménéville, 1843)}

Material examined: Karelia. Kon: 1 female, Konchezero, 24-26. VIII.2011, Polevoi (FRIP); Kol: 1 male, Gizhino, 15.IX.1941, Tiensuu; 1 female, Kolatsel'ga, 9.V.1942, Tiensuu (NHMH); Kton: 1 female, Sukhaya Vodla River, 24.VIII.2006, Polevoi (FRIP).

Published records: Karelia. Kton: Sukhaya Vodla (Humala \& Polevoi 2009, as L. cinctipes (Meigen, 1830). Leningrad Province. Kol: Gumbaritsy (Nartshuk 1999a).

Distribution: Transpalaearctic species.

Biological notes: Dry meadows and agricultural fields. Larvae develop in shoots of different grasses, including cereals rye, wheat, oats, barley, as secondary invaders. In applied literature usually given as $L$. cinctipes due to misidentification.

\section{Lasiosina parvipennis Duda, 1933}

Published record: Karelia. Without locality name (Nartshuk \& Andersson 2013).

Distribution: Eurasian species.

Biological notes: Bogs and marshes.

\section{Melanum laterale (Haliday, 1833)}

Material examined: Murmansk Province. $L p$ : 1 female, Ponoi, Frey (NHMH). Lim: 2 females, Island Berezhnoi Vlasov, 16.VIII.1993; 1 female, Luvenga, 2.IX.1993, Przhiboro (ZISP). Karelia. Kk: 40 males, 13 females, Kartesh, 21-25.VII.1973, 27.VII-16.VIII.1989, Sugonyaev; 15.VII-21.VIII.1996, 3-5.VII.2000, Przhiboro, 22-24. VII.2010, Nartshuk; 1 pupa (reared to adult), Kartesh, 13.VII.1996, Przhiboro; 1 male, 2 females, Island Malyi Andronin, 29.VII.2010, Nartshuk; 1 female, Primorskii, 17.VIII.1996, Przhiboro (ZISP); 7 males, 11 females, Gridino, 5-9.VIII.2006, Polevoi; 7 males, 3 females, Island Luda Lesovata, 9.VIII.2006, Polevoi; 3 females, Sonostrov, 6.VIII.2006, Polevoi; 1 female, Syrovatka, 19.VII.2003, Polevoi (FRIP); Kpoc: 1 female, Island Lodeinyi, 21.VII.2001, Humala; 3 males, 4 females, Island Nemetskii Kuzov, 22.VIII.2002, Humala; 2 females, Island Taparukha, 17.VIII.2002, Humala (FRIP); Kpor: 3 males, 2 females, Island Kondostrov, 20-21.VIII.2002, Humala; 2 males, 6 females, Island Myagostrov, 14.VIII.2002, Humala; 2 males, Perkhludy, Island Yuzhnyi, 16.VIII.2002, Humala (FRIP).

Published records: Karelia. Ks: Paanajärvi (Krogerus 1960); Kon: Shueretskoe (Frey 1934; Nartshuk 1998).

Distribution: Transpalaearctic species.

Biological notes: Wetlands, especially salt marshes along the sea coasts, but also at lake shores and river banks. Not uncommon in the upper intertidal and supralittoral zones (sea shore meadows) of the White Sea. Reared from Juncus gerardii Loisel. (Tschirnhaus 1981; Przhiboro unpubl. data).

\section{Meromyza mosquensis Fedoseeva, 1960}

Material examined: Murmansk Province. Lt: 1 male, 1 female, Murmansk, 19.VIII.1923, Kuznetzov (ZISP). Karelia. Kol: 2 females, Pai, 7.VIII.1923, Kuznetzov (ZISP).

Distribution: Distributed over Europe except the southernmost parts. Biological notes: Meadows. Larvae phytophagous, develop in different species of Poaceae. Hibernate as the third instar larva in shoots of host plants.

\section{*Meromyza nigriseta Fedoseeva, 1960}

Material examined: Karelia. Kl: 1 male, Reuskula, 6.VII.2010, Polevoi (FRIP).

Distribution: Euroasian species, distributed from the British Islands to Malta and Mongolia.

Biological notes: Meadows. Larvae develop in several species of Poaceae, rarely in cereals. 


\section{Meromyza nigriventris Macquart, 1835}

Material examined: Karelia. Kpoc: 1 male, Island Nemetskii Kuzov, 17.VII.2001, Humala; 1 male, 2 females, Island Russkii Kuzov, 18.VII.2001, Humala (FRIP); Kpor: 1 male, Island Bolshoi Zhuzhmui, 23.VII.2001, Humala (FRIP); Kon: 1 male, Raiguba, 17.VII.2002, Polevoi (FRIP).

Distribution: Holarctic species, widely distributed in the Palaearctic from the British Isles to Japan.

Biological notes: Meadows and agricultural fields. Two generations in a year. Larvae phytophagous, develop in different grasses including cereals (wheat, barley, rye). Hibernate as the third instar larvae in shoots of host plants. Namely this species (not M. saltatrix (Linnaeus, 1761)) is a pest of cereals.

\section{Meromyza ornata (Wiedemann, 1817)}

Material examined: Karelia. Kk: 1 female, Kartesh, 16-20.VII.1966, Tanasijtshuk (ZISP); 3 females, Syrovatka, 18-22.VII.2003, Polevoi (FRIP); Kon: 1 female, Lelikovo, 26.VI.2003, Polevoi (FRIP); Kol: 1 male, Sheltozero, 15.VII.2006, Polevoi (FRIP); Kton: 1 male, Sheltoporog, 2.VIII.2002, Polevoi; 2 males, 4 females, Vozritsy, 1.VIII.2002, Polevoi (FRIP).

Published records: Karelia. Kol: Svir', Vidlitsa (Nartshuk 1992, as M. sororcula Fedoseeva 1962).

Distribution: Euroasian species, ranging from the British Isles to Mongolia.

Biological notes: Wet meadows. Larvae phytophagous, live in shoots of Deschampsia cespitosa. The first instar larvae hibernate and in spring move to another plant to continue developing.

\section{Meromyza pluriseta Péterfi, 1961}

Material examined: Murmansk Province. Lim: 1 female, Khibiny, 23.VIII.1928, Cheburova (ZISP). Karelia. Kon: 1 female, Island Bukol'nikov, 25.VI.2003, Polevoi (FRIP).

Published records: Karelia. Kl: Valaam (Nartshuk 1992).

Distribution: Euroasian species, ranging from the British Isles to Mongolia.

Biological notes: Meadows. Larvae develop in shoots of Hierochloa odorata and probably in other Poaceae.

\section{Meromyza pratorum Meigen, 1830}

Material examined: Karelia. Kpoc: 2 males, Island Russkii Kuzov, 18.VII.2001, Humala (FRIP); Kol: 1 male, Lososinnoe, 9-13.VII.2012, Polevoi (FRIP); Leningrad Province. Kol: 1 male, Gimreka, 25.VII.2008, Polevoi (FRIP).

Published records: Karelia. Surroundings of Ladoga Lake (Chydenius \& Furuhjelm 1859); Ks: Paanajärvi (Krogerus 1960).

Distribution: Holarctic species, widely distributed in the Palaearctic from the British Isles to Japan.

Biological notes: Dry meadows and sandy dunes on the sea coasts. Larvae phytophagous, develop in shoots of Calamagrostis epigejos and Ammophila arenaria (L.) Link.

\section{Meromyza saltatrix (Linnaeus, 1761)}

Material examined: Murmansk Province. $L t$ : 3 males, Murmansk, 23.VIII.1928, Kuznetzov (ZISP). Karelia. Kk: 2 males, 1 female, Kartesh, 16-20.VII.1966, Tanasijtshuk (ZISP); 1 male, Gridino, 14.VII.2007, Humala (FRIP); Kpor: 1 male, Island Bolshoi Zhuzhmui, 23.VII.2001, Humala (FRIP); Kon: 3 males, 3 females, Island Lambaznik, 25.VI.2003, Polevoi; 1 female, Lelikovo, 26.VI.2003, Polevoi (FRIP); Kol: 1 male, Mayachino, 22.VI.2012, Polevoi (FRIP); 1 male, Pai, 7.VIII.1923, Kuznetzov (ZISP).

Published records: Murmansk Province. Kandalaksha (Nartshuk 1992). Karelia. Surroundings of Ladoga Lake (Chydenius \& Furuhjelm 1859); Kрос: Vuokkiniemi; Kon: Konchezero; Kl: Valamo, Impilahti, Kirjavalahti (Nartshuk 1992).

Distribution: Holarctic species, widely distributed in the Palaearctic. Biological notes: Meadows. Larvae phytophagous, in different grasses (Poaceae). Hibernate as the third instar larva. The species has been recorded as a pest of cereals by different authors, but in fact, it does not live in cereals, all these records refer to $M$. nigriventris.

\section{Meromyza triangulina Fedoseeva, 1960}

Material examined: Karelia. Kol: 1 female, Shoksha, 13.VII.2004, Polevoi (FRIP).

Published records: Karelia. Kon: Tivdia (Nartshuk 1992).

Distribution: European species.

Biological notes: Meadows. Probably two generations in a year. Larvae phytophagous, develop in different grasses (Poaceae), especially Festuca spp. Hibernate as the third instar larva in shoots of host plants.

\section{Neohaplegis tarsata (Fallén, 1820)}

Material examined: Karelia. Kk: 1 male, Syrovatka, 17.VII.2003, Polevoi (FRIP); Kl: 1 female, Impilahti, Forsius (NHMH); Kon: 1 female, Vottovaara Mountain, 18.VII.2008, Polevoi; 1 male, Tipinitsy, 4.VII.2004, Polevoi (FRIP); Kpor: 1 female, Valdai, 29.VI.2010, Polevoi (FRIP).

Distribution: Transpalaearctic species.

Biological notes: Wetlands with sedges. Larvae live in shoots of Carex spp.

\section{Platycephala planifrons (Fabricius, 1798)}

Material examined: Karelia. Kl: 3 females, Salmi, Tuomikoski (NHMH), 29.VII.1994, Humala (FRIP); 1 female, Jakkima, Sahlberg (NHMH); 1 female, Ladoga Lake, Nordqvist (NHMH). Kpor: 2 females, Island Myagostrov, 14.VIII.2002, Humala (FRIP).

Published records: Karelia. Surroundings of Ladoga Lake (Chydenius \& Furuhjelm 1859); Kon: Shueretskoe (Nartshuk 1999a).

Distribution: Euroasian species, distributed from the British Isles to Mongolia.

Biological notes: Common reed (Phragmites australis) beds. Larvae phytophagous, live in stems of common reed, destroying the vegetation point with the result that the upper leaves wilt. 


\section{Pseudopachychaeta approximatonervis (Zetterstedt, 1848)}

Material examined: Karelia. Kon: 1 female, Solomennoe, Sahlberg (NHMH); Kl: 1 male, Yakkima, Forsius (NHMH); Kol: 1 male, Petrozavodsk, Sahlberg (NHMH).

Published records: Karelia. Surroundings of Ladoga Lake (Chydenius \& Furuhjelm 1859); Ks: Paanajärvi (Krogerus 1960).

Distribution: Holarctic species.

Biological notes: Wetlands. Larvae phytophagous, live in inflorescences of Eriophorum spp. except E. vaginatum L., feeding on unripe seeds. Hibernate as adults.

\section{Pseudopachychaeta ruficeps (Zetterstedt, 1838)}

Material examined: Murmansk Province. Lps: 1 male, Trifonovo, Hellen (NHMH); Lt: 5 males, 3 females, Kola, Frey; 1 male, Polyarnyi, Frey (NHMH); Lim: 1 male, 1 female, Belaya Guba, Frey (NHMH); Lim: 4 males, 2 females, Lake Bolshoi Vudjavr, 14.IX.1930, 18.VI-24.VIII.1931, Fridolin; 3 males, 1 female, Khibiny, 7-28.VIII.1928, Cheburova; 1 female, Island Berezhnoi Vlasov, 16.VIII.1993, Przhiboro (ZISP); 1 male, Lisii Stream, 28.V.2014, Polevoi (FRIP); 1 male, Kandalaksha, Hellen (NHMH); Lp: 3 males, Ponoi, Frey (NHMH). Karelia. Kk: 2 females, Kartesh, 20.VIII.1996, 4.IX.2005, Przhiboro (ZISP); Kon: 3 females, Shaidoma, 10-11. VIII.2018, Polevoi; 1 female, Vendery, 30.VIII.2017, Polevoi; 1 male, 3 females, Kivach, 2.IX.2002, 1.VIII.2003, Polevoi (FRIP); Kol: 1 male, Sheltozero, 15.VII.2006, Polevoi (FRIP).

Published records: Murmansk Province. Ks: Kuolajärvi. Karelia. Ks: Paanajärvi (Nartshuk 1999a).

Distribution: Transpalaearctic arcto-boreal species.

Biological notes: Bogs. Females lay eggs early in spring into inflorescences of Eriophorum vaginatum, where phytophagous larvae then develop. Up to 4-6 larvae may live in one inflorescence. Adults appear in July and hibernate.

\section{Thaumatomyia glabra (Meigen, 1830)}

Material examined: Murmansk Province. Lmur: 1 male, Gavrilovo, Enwald River (NHMH). Karelia. Kk: 3 males, 2 females, Kartesh, 9-16.VIII.1989, Sugonyaev (ZISP), 28.VII.1996, Polevoi (FRIP); 17-26.VII.2010, Nartshuk (ZISP); 6 males, 9 females, Gridino, 7.VIII.2007, Polevoi, Humala, 14.VII.2007, Humala (FRIP); Kpor: 3 females, Island Myagostrov, 14.VIII.2002, Humala; 1 female, Island Pechak, 24.VII.2001, Humala (FRIP); 2 females, Segezha, 29.VII.1996, Gorodkov (ZISP); Kb: 1 female, Tolvojärvi, 27.VII.1998, Polevoi (FRIP); Kon: 1 male, 4 females, Shaidoma, 10-11.VIII.2018, Polevoi; 7 males, 2 females, Lisitsyno, 4.VII.2004, Polevoi; 1 male, Island Bukol'nikov, 25.VI.2003, Polevoi (FRIP); 1 female, Konchezero, 7.VII.1942, Tiensuu (NHMH); Kl: 1 female, Sukopohja, 7.VII.2005, Polevoi (FRP); Kol: 1 male, 1 female, Petrozavodsk, Sahlberg, Tiensuu (NHMH); Kton: 1 female, Kolovo, 14.VI.2003, Polevoi (FRIP).

Published records: Karelia. Kpoc: Segozero (Frey 1934).

Distribution: Holarctic species.

Biological notes: Abundant everywhere and found in a variety of habitats. Larvae carnivorous, live between the plant roots, feeding on root aphids. The fly larvae occur between the root aphids and their white wax, partly also with the Lasius flavus ants, which visit the aphids to collect their sugar excretions or the aphids themselves (M. von Tschirnhaus, pers. comm.).
Thaumatomyia hallandica Anderson, 1966

Material examined: Karelia. Kk: 3 males, 2 females, Kartesh, 20.VII.1966, Tanasijtshuk, 23.VII.1975, Gorodkov, 20.VIII.1996, Przhiboro (ZISP); Kpoc: 1 female, Island Lodeinyi, 21.VII.2001, Humala; 1 female, Island Nemetskii Kuzov, 17.VII.2001, Humala; 8 males, 14 females, Island Russkii Kuzov, 18-19.VII.2001, Humala (FRIP); Kpor: 1 female, Island Bol'shoi Zhuzhmui, 23.VII.2001, Humala; 2 females, Island Kondostrov, 20.VIII.2002, Humala; 3 males, 5 females, Perkhludy, Island Yuzhnyi, 16.VIII.2002, Humala (FRIP); Kpor: 1 male, Segezha, 29.VII.1996, Gorodkov (ZISP); Kon: 2 males, 2 females, Lisitsyno, 4.VII.2004, Polevoi; 1 female, Lelikovo, 25.VI.2003, Polevoi; 1 female, Island Radkol'e, 25.VI.2003, Polevoi; 1 male, 2 females, Konchezero, 8.VII.1942, Tiensuu (NHMH), 24-26. VIII.2011, 4-6.VII.2012, Polevoi (FRIP); Kol: 1 male, Sheltozero, 29.VIII.1942, Tiensuu (NHMH).

Published records: Karelia. Kol: Petrozavodsk; Kpoc: Segozero (Frey 1934); Kk: Chupa (Nartshuk 1999a).

Distribution: Eurasian species, distributed from the British Isles to Mongolia and Central Asia.

Biological notes: Dry meadows and sandy dunes. Larvae carnivorous, live between plant roots, feeding on root aphids. Prefers permanent wet ground (Festucetum rubrae litoralis, Puccinellietum maritimae in salt marshes, also wet grass stands along small high alpine streams). In dune systems they fly around but prefer the wet depressions (M. von Tschirnhaus, pers. comm.).

\section{Thaumatomyia notata (Meigen, 1830)}

Material examined: Murmansk Province. Lim: 10 males, 19 females, Island Ryashkov, 5.IX.1984, Gorodkov (ZISP). Karelia. Ks: 1 male, Paanajärvi, Platonoff (NHMH); Kk: 1 male, Kartesh, 20.VII.2010, Nartshuk (ZISP); 1 female, Gridino, 14.VII.2007, Humala (FRIP); Kpor: 1 female, Segezha, 29.VII.1996, Gorodkov (ZISP); Kon: 1 female, Medvezh'egorsk, 7.VI.2000, Polevoi; Kon: 2 females, Shaidoma, 10.VIII.2018, Polevoi; 1 female, Lisitsyno, 4.VII.2004, Polevoi; 1 male, 1 female, Kondopoga, 30.VIII-6.IX.2011, Kainelainen; 1 female, Konchezero, 4.VI.2013, Polevoi (FRIP); Kl: 1 female, Salmi, Woldstedt (NHMH); Kol: 2 males, 1 female, Petrozavodsk, Günther, Tiensuu (NHMH), 17.V.2019, Polevoi (FRIP); 5 males, 12 females, Ust'e Tuloksy, 5.IX.2018, Polevoi; 1 female, Shoksha, 13.VII.2004, Polevoi; 1 male, Sheltozero, 14-15.VII.2006, Polevoi (FRIP); Kton: 1 female, Sukhaya Vodla River, 7.VI.2002, Polevoi (FRIP); Kp: 1 female, Schanikovskaya, 23.VI.2009, Humala (FRIP).

Published records: Karelia. Kl: Sortavala (Nartshuk 1999a).

Distribution: Palaearctic, Afrotropical and Oriental regions. In the Palaearctic, distributed from the British Isles to Japan.

Biological notes: Found in a variety of habitats, often visit flowers. Larvae carnivorous, live between the plant roots, feeding on root aphids. Hibernate as adults. Flies aggregate before hibernation (sometimes up to several millions of specimens), in autumn may penetrate into houses. This phenomenon was recorded nearly every 10-11 years (Nartshuk 2000; Kotrba \& Nartshuk 2008).

\section{Thaumatomyia rufa (Macquart, 1835)}

Material examined: Karelia. Kpoc: 1 female, Belomorsk, Sahlberg; 1 female, Shueretskoe, Sahlberg (NHMH); Kon: 1 female, Shaidoma, 11.VIII.2018, Polevoi; 3 females, Island Lambaznik, 25.VI.2003, 
Polevoi; 1 female, Lelikovo, 25.VI.2003, Polevoi (FRIP); 2 males, Konchezero, 6.VII.1942, Tiensuu (NHMH); Kl: 1 male, Sortavala, 30.VII.1935, Tiensuu; 1 male, Salmi, 7.VIII.1941, Tiensuu (NHMH); Kol: 1 male, Kolatsel'ga, 7.VIII.1943, Tiensuu; 1 male, 1 female, Petrozavodsk, Sahlberg (NHMH).

Distribution: Transpalaearctic species, distributed from the British Isles to Japan.

Biological notes: Meadows. Larvae carnivorous, live between the plant roots, feeding on root aphids.

\section{Thaumatomyia trifasciata (Zetterstedt, 1848)}

Material examined: Murmansk Province. Lps: 1 female, Borisoglebskii, Hellen; 1 female, Kuvernöörikoski, Hellen; 1 female, Trifonovo, Hellen; 2 males, 3 females, Yläluostari, 8.VII.1929, Lindberg (NHMH); 3 females, Langvatn, 4.VIII.2008, Humala (FRIP); 2 females, Lotta River, Platonoff; $L t: 3$ females, Polyarnyi, 17.VI.1926, Barovskii (ZISP), Hellen (NHMH); 1 female, TyuvaGuba, 23.VIII.1923, Fridolin; 1 female, Murmansk, 15.VI.1910, Fedotov (ZISP); 2 females, Kola, Hellen; 1 male, $50 \mathrm{~km} \mathrm{~W}$ of Verhnetulomskii, 9.VIII.1967, Meinander (NHMH); Lmur: 2 males, Gavrilovo, Hellen (NHMH); 1 female, Tumannyi, 14.VII.1974, Kasparyan (ZISP); 1 male, 2 females, Voroninsk, Palmen (NHMH); 1 female, Lake Seidozero, 31.VII.1974, Kasparyan (ZISP); Lim: about 20 specimens, Island Telyachii and Luvenga, VII-VIII.1987-1993, Przhiboro; 1 male, 1 female, Island Berezhnoi Vlasov, 16.VIII.1993, Przhiboro (ZISP); 12 females, Monchegorsk, 20-24.VII.1995, Kozlov (ZISP); 1 male, Belaya Guba, Frey (NHMH); 2 females, Lake Malyi Vudjavr, 4-30.VII.1974, Kasparyan; 7 females, Lake Bolshoi Vudjavr, 14.IX.1930, 30.VI.1931, 9-25.VII.1934, Fridolin; 6 females, Yuksporrjok River, 24.VII.1974, Kasparyan; 2 females, Khibiny, 17.VII.1926, Barovskii (ZISP); 2 females, Kandalaksha, Frey \& Hellen (NHMH); 1 female, Kolvitsa, 9.VIII.1995, Gorodkov (ZISP); $L p$ : 1 male, 2 females, Ponoi, Frey (NHMH). Karelia. Ks: 1 male, Nuorunen Mountain, 12.VII.1990, Jakovlev (FRIP); Kk: 1 male, 2 females, Primorskii, 17.VIII.1996, Przhiboro; 1 female, Nikol'skaya Bay, 28.VII.1996, Przhiboro; 22 males, 35 females, Kartesh, 16-20. VII.1966, Tanasijtshuk, 23.VII.1975, Gorodkov, 6.VIII.1982, Zaitzev, 27.VII-16.VIII.1989, Sugonyaev, 14.VII.1992, 4.VIII-21.VIII.1996, 18.VI.1997, 28.VI-23.VII.2000, 16-27.VI.2002, 4.IX.2005, Przhiboro, 20-24.VII.2010, Nartshuk; 2 larvae, 17 pupae (reared to adults), 21.VIII.1996，2.X.1996，6-8.VII.2000， 3.X.2003， 22.V.2004, 4.IX.2005, Kartesh, Przhiboro (ZISP); 20-25.VII.1996, Polevoi (FRIP); 2 females, Keret', 13.VIII.1966, Tanasijtshuk (ZISP); 1 female, Chupa, 30.VI.2000, Przhiboro (ZISP); 11 females, Gridino, 7.VII-5. VIII.2007, Polevoi; 1 female, Island Pezhostrov, 7.VIII.2006, Polevoi; 1 female, Sonostrov, 6.VIII.2006, Polevoi; 23 males, 45 females, Syrovatka, 16-22.VII.2003, Polevoi (FRIP); Kpoc: 3 females, Lake Levi, 4.VII.1998, Polevoi; 2 females, Ladvozero, 11.VII.1996, Polevoi; 1 female, Nesterova Mountain, 23.VI.2000, Polevoi; 2 females, Island Lodeinyi, 21.VII.2001, Humala; 7 females, Island Nemetskii Kuzov, 17.VII.2001, 22.VIII.2002, Humala; 1 female, Island Russkii Kuzov, 18.VII.2001, Humala (FRIP); Kpor: 1 female, Island Myagostrov, 14.VIII.2002, Humala (FRIP); 2 males, 4 females, Segezha, 4.VII.1921, Olonets Expedition, 29.VII.1996, Gorodkov (ZISP); Kon: 1 male, 5 females, Shaidoma, 10-11.VIII.2018, Polevoi; 1 male, 3 females, Lake Shuyal, 8-9.VII.2006, Polevoi; 1 male, 2 females, Kurgenitsy, 18.VII.2000, Polevoi; 1 male, 4 females, Konchezero, 24-26.VIII.2011, 4.VII.2012, Polevoi (FRIP); Kl: 1 male, Salmi, Westerlund (NHMH); Kol: 2 males, 2 females, Vidlitsa, 4.IX.2018, Polevoi; 1 female, Ust'e
Tuloksy, 5.IX.2018, Polevoi; 3 females, Lososinnoe, 9-13.VII.2012, Polevoi (FRIP); 1 female, Petrozavodsk, Günther; 1 female, Olonets, 27.VII.1942, Tiensuu (NHMH); 2 females, Sheltozero, 15.VII.2006, Polevoi (FRIP); Kton: 2 females, Shoikapolda River, 22.VIII.2006, Polevoi; 1 female, Vozritsy, 1.VIII.2002, Polevoi (FRIP).

Published records: Murmansk Province. Lps: Pechenga, Vuoremijärvi; Ks: Kuolajärvi (Nartshuk 1999a). Karelia. Ks: Paanajärvi; Kk: Chupa; Kpoc: Segozero (Krogerus 1960; Nartshuk 1999a).

Distribution: Holarctic arcto-boreal species.

Biological notes: Wetlands and the sea intertidal zone (mostly tidal meadows). Larvae carnivorous, live between the plant roots, feeding on root aphids. The only Thaumatomyia species, which is common at the White Sea shores as adults. Larvae of only this Thaumatomyia species are common in the upper intertidal zone of the White Sea, where they are associated mostly with the aphid species Colopha compressa (Koch, 1856) and, more rarely, with Pemphigus bursarius (Linnaeus, 1758) (Przhiboro unpubl. data).

\section{Distribution and Zoogeography}

The study region is extending for nearly 10 degrees from the southern border of Karelia to the coast of the Arctic Ocean and basically lies in the limits of Southern, Middle and Northern boreal vegetation zones. Boreal forests cover most of this territory leaving only a relatively narrow stripe along the Barents Sea coast occupied by tundra. This territory was covered by glaciation during the Riss period and recolonization started after the retreat of the glaciers, nearly 10-12 thousand years ago. The main route of colonization was from south to north. Certain species have acclimatized in different parts of the territory probably due to individual tolerance to the climatic condition, first of all, to low temperature.

In the boreal zone, only a few species of Chloropidae are associated with forests. Larvae of the genus Gaurax develop in rotting wood, larvae of Hapleginella laevifrons, in damaged coniferous cones. Tricimba cincta is often reared from fungi. There are no exact data on food substrates of these species, but these may include mycelia, macerated wood or dead insects occurring in the same microhabitats. Other species of Chloropidae occurring in the boreal zone inhabit different meadows, forest edges, and clearings with grasses as well as bogs, fens and marshes with sedges.

Northern melanism is known in some light-coloured species of Chloropidae occurring on the Kola Peninsula. Specimens of Gaurax, especially females, collected in northern localities, have nearly all body black. Specimens of Chlorops speciosus, C. meigenii, Diplotoxa messoria, Thaumatomyia trifasciata, Pseudopachychaeta ruficeps from northern localities have their black stripes on the thorax wide and nearly fused, basal segment of antennae black and legs partly black.

The recent fauna of Chloropidae of Karelia and Murmansk Province includes species having various range types: species with multiregional distribution, including Afrotropical and/or Oriental regions besides the Palaearctic, account for 3.7\%, Holarctic species $16.5 \%$, Transpalaearctic boreal - 24.8\%, Eurasian - 33\%, European (including Euro-Caucasian-Kazakhstanian) - 22\%. A higher percentage of Holarctic species in the regional fauna (16.5\%) in comparison with that in the fauna of the entire Palaearctic (no more than $10 \%$ ) agrees well with the zoogeographical structure of the Chloropidae fauna in other northern countries.

The distribution of frit flies in the study territory is still inadequately known; many species are recorded only from one or two 
localities. Probably some of them are rare or stenotopic, e.g. Incertella karteshensis, Chlorops centromaculatus, C. serenus, Diplotoxa dalmatina, Lasiosina albipila, Eribolus hungaricus, E. slesvicensis, Gaurax leucarista, G. venustus, Calamoncosis oscinella (the three last ones are known only from one-two localities in the other parts of Europe). The remaining species may appear to be more common in the future. All recorded species can be tentatively divided into four main groups characterized by different distribution patterns according to the northern border of their ranges on the study territory. These groups may be characterized as follows.

1. Widely distributed species recorded from the whole study territory, including the extreme North. These are species with the widest, holarctic and transpalaeartic boreal ranges. Many of them (e.g., most of the phytophagous species of the subfamily Chloropinae and even Thaumatomyia spp. with carnivorous larvae) are associated predominantly with Cyperaceae. Most of these species reach the northern limits of the Northern Boreal zone but some (Oscinella frit, Chlorops planifrons, C. scutellaris, Melanum laterale, Pseudopachychaeta ruficeps, Thaumatomyia trifasciata) occur in the tundra zone as well (Nartshuk 2005).

The following species belong to this group. Subfamily Oscinellinae: Aphanotrigonum trilineatum, Conioscinella frontella, C. livida, Elachiptera cornuta, E. diastema, Hapleginella laevifrons, Incertella albipalpis, I. kerteszi, I. nigrifrons, Microcercis trigonella, Oscinella frit, O. nitidissima, Polyodaspis ruficornis, Rhopalopterum atricillum, R. femorale, Siphonella oscinina, Tricimba cincta. Subfamily Chloropinae: Cetema cereris, Chlorops meigenii, C. planifrons, C. rossicus, C. scutellaris, C. speciosus, C. troglodytes, Melanum laterale, Meromyza mosquensis, M. saltatrix, Pseudopachychaeta ruficeps, Thaumatomyia glabra, T. trifasciata.

2. Species widely distributed in Karelia, but reaching the southern border of Murmansk Province at the most. With some exceptions, this assemblage includes common meadow species predominantly associated with grasses (Poaceae). In more northern localities, these species are gradually substituted by wetland species associated with sedges (Cyperaceae). Subfamily Oscinellinae: Aphanotrigonum nigripes, Conioscinella sordidella, Elachiptera tuberculifera, Eribolus nana, Oscinella cariciphila, O. pusilla, Dicraeus fennicus. Subfamily Chloropinae: Chlorops limbatus, C. hypostigma, C. varsoviensis, C. geminatus, C. figuratus, C. pumilionis, Diplotoxa messoria, Meromyza ornata, M. pratorum, Neohaplegis tarsata, Pseudopachychaeta approximatonervis, Thaumatomyia hallandica, T. notata.

3. Species distributed up to the northern border of the biogeographical province Karelia pomorica occidentalis (approximately the latitude of the Solovetsk Islands). This group includes a few species associated with cereals and one species associated with reed (Phragmites australis), its northern border accords well with the northern border of the host plant range (Hultén 1971). Subfamily Oscinellinae: Lasiambia palposa, Oscinella nigerrima. Subfamily Chloropinae: Platycephala planifrons, Meromyza nigriventris, Chlorops scalaris, C. hypostigma, Thaumatomyia rufa.

4. Species found only in the southern part of Karelia, reaching approximately the northern border of the Boreonemoral zone. This group includes species with European, Euro-Caucasian and EuroMediterranean ranges possibly representing a nemoral element of the fauna. Although some of these species are found only in one or two localities in southern Karelia, they all are included here based on

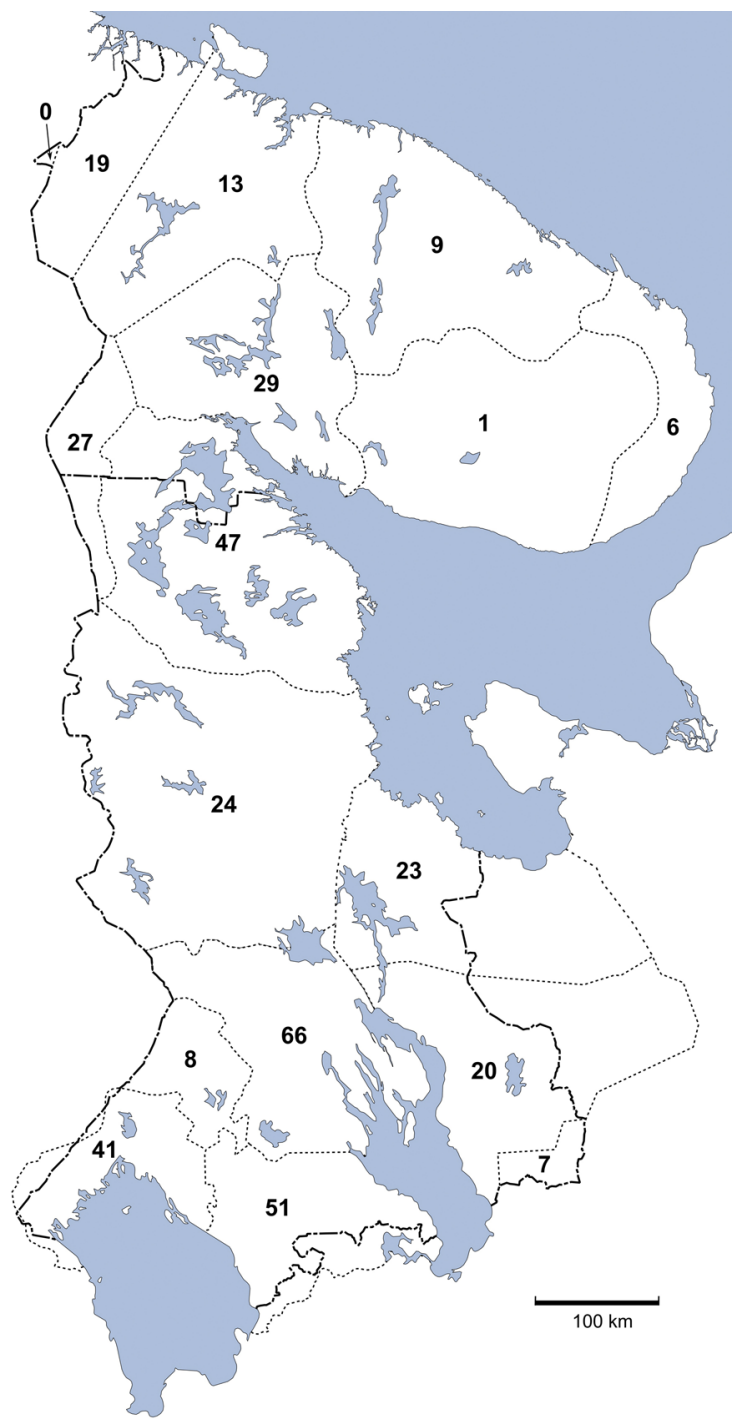

Figure 2. The number of Chloropidae species recorded in biogeographical provinces of the Russian part of Fennoscandia.

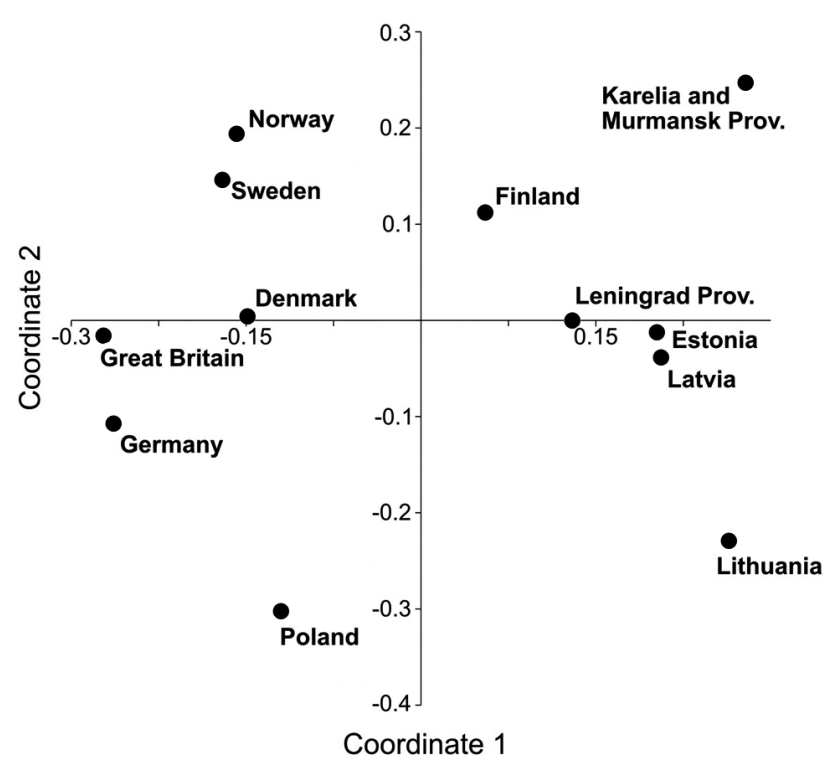

Figure 3. NMDS diagram (stress $=0.15$ ), showing similarity (Dice measure) between the Chloropidae fauna of north-European regions. 
their distributions in the neighboring regions. Subfamily Oscinellinae: Aphanotrigonum cinctellum, Calamoncosis aprica, Conioscinella gallarum, C. mimula, Oscinella maura, O. trochanterata, Speccafrons halophila, Trachysiphonella ruficeps, T. scutellata. Subfamily Chloropinae: Cetema simile, Chlorops anthracophagoides, C. frontosus, C. gracilis, C. laetus, C. ringens, Lasiosina herpini, Meromyza nigriseta, M. triangulina.

As in many other groups of insects, the number of Chloropidae species in Fennoscandia gradually decreases northwards (Nartshuk \& Andersson 2013). In Karelia and Murmansk Province this trend is less distinct due to unequal knowledge of the territory (Figure 2), however, the species composition is significantly impoverished north of latitude $64-65^{\circ} \mathrm{N}$, which approximately coincides with the northern border of the Middle boreal zone. Hence, a large number of species reaches the northern limit of their distribution in Karelia while only 38 species (35.2\%) were found north of the Arctic Circle $\left(66^{\circ} 33^{\prime} \mathrm{N}\right)$. It is clearly seen that species occurring throughout Karelia and those reaching the northern part of the Kola Peninsula mainly have wide holarctic and transpalaearctic ranges, while species recorded only in the southern part of Karelia mostly have a European and Euro-Mediterranean distribution.

The similarity between the Chloropidae fauna of the northEuropean regions is displayed in the NMDS diagram (Figure 3), which almost perfectly mirrors the geographical position of the countries under comparison. The first and second axes in this case approximately coincide with the directions South-North and WestEast. The Russian part of Fennoscandia (Karelia and Murmansk Province) is more similar to Leningrad Province, Estonia, Latvia and Finland. This result indicates that the study territory has been recolonized mainly from the south and partly from the southeast. Thus, the species groups discussed above reflect the rate of colonization by different species and, probably, their tolerance to low temperatures. Only the following species are most likely of eastern origin: Polyodaspis ruficornis, Chlorops kirigaminensis and Lasiosina parvipennis. They are common and abundant in the East Palaearctic but are rather rare or absent in the western part of Europe. On the other hand, Norway and especially Sweden share many species with Germany and Great Britain. Such southern elements probably penetrated into the Scandinavian Peninsula via Denmark and, to date, they are not expanding further than its southern part (Aphanotrigonum inerme Collin, 1946, Dicraeus raptus (Haliday, 1838), Gaurax flavomaculatus (Duda, 1933), Incertella antennata (Collin, 1946), Oscinimorpha albisetosa (Duda, 1932), Oscinisoma gilvipes (Loew, 1858), Chlorops dasycerus Loew, 1866, Eurina lurida Meigen, 1830, Meromyza bohemica Fedoseeva, 1962, M. femorata Macquart, 1835, Parectecephala longicornis (Fallén, 1820)). Some of the southern (nemoral) species are recorded only on islands (Öland, Gotland, Bornholm): Dicraeus raptus, D. ingratus (Loew, 1866), Chlorops finitimus Becker, 1910, C. pallidiventris (Duda, 1933), C. pannonicus Strobl, 1893, Chloropsina distinguenda (Frey, 1909), C. rohaceki Nartshuk, 2000, Neohaplegis glabra Duda, 1933. These species are probably a remnant of the fauna of the interglacial xerothermic period and preserved due to a warmer present-day climate on islands compared to the mainland Scandinavia. Finland occupies an intermediate position on the diagram and is situated a little closer to the "eastern" group of countries. This may indicate the primarily south-eastern origin of its fauna with an admixture of south-western species.

A number of species has been described from the northern parts of Fennoscandia. Probably they are examples of relatively late speciation in the northern boreal zone: Conioscinella abiskoi Nartshuk \& Andersson, 2013, C. tornensis Nartshuk \& Andersson, 2013, C. messaurea Nartshuk \& Andersson, 2013, Aphanotrigonum norrbotticum Nartshuk \& Andersson, 2013 (from northern Sweden) and Incertella karteshensis (from northern Karelia). Two dendrophilous species of Gaurax demonstrate a curious pattern of distribution: $G$. venustus is associated with aspen wood and found in Karelia and $G$. flavomaculatus is associated with broad-leaved trees and found in Norway (Nartshuk, 2008). Both species are not recorded in adjacent territories of Fennoscandia but are known from Central Europe (Austria and Hungary, Germany and Switzerland, respectively). However, taking into account the principal rarity of Gaurax spp., these species may really be more widely distributed.

\section{ACKNOWLEDGEMENTS}

The authors sincerely acknowledge the help of administration and staff of protected areas (Pasvik Nature Reserve, Laplandskii Nature Reserve, Kivach Nature Reserve, Vodlozerskii National Park, Kandalaksha State Reserve) during collecting in appropriate territories. We sincerely acknowledge the help of Jevgeni Jakovlev and Jere Kahanpää (Helsinki, Finland), who kindly provided us with the information on some poorly known species stored in the collection of Natural History Museum, Helsinki. We also thank Juho Paukkonen (Helsinki, Finland), who helped to clarify position of uncertain collecting localities visited by Finnish scientists in Murmansk Province. We greatly appreciate valuable comments on the manuscript by anonymous referee and by Michael von Tschirnhaus. The work of E.P. Nartshuk was performed in the frame of the State Research Project AAAA-A19-119020690082-8. The work of A. Przhiboro was performed in the frames of the State Research Project AAAA-A19-119020690091-0 and was partially supported by the Russian Foundation for Basic Research (grant no. 18-04-00988). The work of A. Polevoi was carried out under the state order to the Karelian Research Centre of the Russian Academy of Sciences (Forest Research Institute KRC RAS).

\section{REFERENCES}

Ahti T, Boychuk M. 2006. The botanical journeys of A.K. Kajander and J.I. Lindroth to Karelia and Onega river in 1898 and 1899, with a list of their bryophyte and lichen collections. Norrlinia 14: 1-65.

Bährmann R. 1985. Untersuchungen der Diversität und Stabilität der Dipterenfauna einer naturnahen und einer anthropogen beeinflussten Rasenkatena bei Jena/Türingen. Zoologische Jahrbücher. Abteilung für Systematik, Ökologie und Geographie der Tiere 112(2): 235-248.

Brauns A. 1938. Die Flügelrückbildung bei Strandfliege Conioscinella brachyptera Zett. und die Beziehungen zur Ausbildung der Flügelsinneskuppeln. Zoologischer Anzeiger 123: 281-295.

Chandler P. 1998. Checklists of insects of the British Isles (New Series). Part 1 (incorporating a List of Irish Diptera): Diptera. Chloropidae. Handbooks for the Identification of British Insects 12: 145-149.

Cherian PT. 2012. Revision of some genera of tribe Fiebrigellini and description of nine new species of Anacamptoneurum Becker from the Oriental Region (Diptera: Chloropidae: Oscinellinae). Acta zoologica Cracoviensia 55(1): 1-32. doi: 10.3409/azc.55 1.01.

Chydenius JJ, Furuhjelm JE. 1859. Berättelse öfver en naturhistorisk resa i Karelen, företagen på Sällskapets pro Fauna et Flora fennica bekostnad [med en anteckning af W. Nylander]. Notiser ur Sällskapets pro Fauna et Flora fennica Förhandlingar 4(1858-1859): 79-118.

Dabrowska-Prot E. 1984. Structural and functional characteristics of Chloropidae community in an industrial landscape. Polish Ecological 
Studies 10(1): 111-140.

Duda O. 1933. 61. Chloropidae. In: Lindner E. (ed). Die Fliegen der Palaearktischen Region, 6(1). Stuttgart. E. Schweizerbart'sche Verlagsbuchhandlung. pp 49-248.

Ebejer M, Andrade R. 2015. The Chloropidae (Diptera, Brachycera) of mainland Portugal with description of a new species of Lasiosina Becker. Entomologist's monthly Magazine 151: 227-271.

Ebejer MJ, Kettani K. 2016. An overview of the Chloropidae (Diptera) of Morocco with new records, description of a new species of Pselaphia Becker and an updated list of species. Entomologist's monthly Magazine 125: 225-244

El-Hassani A, Messaoudi J. 1986. Les ravageurs des cônes et graines de conifères et leur distribution au Maroc. In: Roques A. (ed). Proceedings of the 2nd conference of the cone and seed insects working party S2.0701, IUFRO, Briançon, France, September 3-5, 1986. Ardon, Olivet. Station de Zoologie Forestière, I.N.R.A. pp 5-14.

Ferrar P. 1987. A guide to the breeding habits and immature stages of the Diptera Cyclorrhapha. Part 1. Entomonograph 8: 9-478.

Frey R. 1934. Förteckning över Finlands Chloropider, bestämda av O. Duda. Memoranda Societatis pro Fauna et Flora Fennica 9: 128-139.

Gaidene EK, Nartshuk EP. 1963. On the biology of Hapleginella laevifrons Lw. (Diptera, Chloropidae) the cone pest. Entomological Review 42: $411-413$.

Grochowska M. 2002. Remarks on morphology of immature stages, biology and life cycle of Calamoncosis aprica (Meigen, 1830) (Diptera, Chloropidae). Mitteilungen aus dem Museum für Naturkunde in Berlin - Deutsche entomologische Zeitschrift 49(2): 279-290. doi: 10.1002/ mmnd.20020490212.

Grochowska M. 2006. Morphology of preimaginal stages of Lipara pullitarsis Doskočil \& Chvála, 1971 (Diptera: Chloropidae) — a gallforming fly in the common reed (Phragmites australis). Entomologica Fennica 17(4): 387-393. doi: 10.33338/ef.84362.

Grochowska M. 2007. Morphology of preimaginal stages of Lipara rufitarsis Loew 1858 (Diptera: Chloropidae), a parasite of the common reed (Phragmites australis (Cav.) Trin.). Annales de la Société Entomologique de France 43(1): 57-62. doi: 10.1080/00379271.2007.10697494.

Haarto A, Kakko I, Winqvist K. 2019. Lisäyksiä Suomen Diptera-faunaan vuoden 2014 jälkeen. W-album 22: 3-31.

Hammer Ø, Harper DAT, Ryan PD. 2001. PAST: Palaeontological statistics software package for education and data analysis. Palaeontologica electronica 4(1): 1-9.

Heikinheimo O, Raatikainen M. 1971. The recording of locations of biological finds in Finland. Annales entomologici Fennici 37(1a): 1-27.

Hultén E. 1971. Atlas över växternas utbredning i Norden: fanerogamer och ormbunksväxter. Stockholm. Generalstabens Litografiska Anstalt $587 \mathrm{p}$.

Humala AE, Polevoi AV. 2006. Composition and structure of the insect communities in the forests formed on various soil-forming bedding rocks. In: Fedorets NG. (ed). Soil diversity and biodiversity in the middle taiga ecosystems. Moscow. Nauka. pp 67-92. (In Russian).

Humala AE, Polevoi AV. 2008. Insects. In: Gromtsev AN. (ed). Rupestrian landscapes of the White Sea Karelian coast: natural characteristics, economic utilization, conservation. Petrozavodsk. Karelian Research Centre RAS. pp 125-136. (In Russian).

Humala, AE, Polevoi AV. 2009. On the insects fauna of South-East Karelia. Transactions of Karelian Research Centre of the Russian Academy of Sciences 9(4): 53-75. (In Russian).

Ismay JW. 1980. Recent records of Eribolus (Dipt., Chloropidae) Entomologist's monthly Magazine 115: 96.

Ismay JW. 1985. The identity of Cetema elongata (Meigen) (Dipt., Chloropidae). Entomologist's monthly Magazine 121: 35-38.

Ismay JW. 1991. Dicraeus tibialis (Macquart) (Diptera: Chloropidae) new to New Zealand. New Zealand Entomologist 14: 1-24. doi: 10.1080/00779962.1991.9722607.

Ismay JW, Nartshuk EP. 2000. Family Chloropidae. In: Papp L, Darvas B. (eds). Contribution to a Manual of Palaearctic Diptera. Appendix.
Budapest. Science Herald. pp 387-430.

Jakovlev J, Polevoi A, Humala A. 2014. Insect fauna of Zaonezhye Peninsula and adjacent islands. In: Lindholm T, Jakovlev J, Kravchenko A. (eds). Biogeography, landscapes, ecosystems and species of Zaonezhye Peninsula, in Lake Onega, Russian Karelia. Helsinki. Finnish Environment Institute. pp 257-338.

Kailidis DS, Georgevits RP. 1972. Forstinsekten Griechenlands. Tanneninsekten. Anzeiger für Schädlingskunde und Pflanzenschutz 45(2): 25-28. doi: 10.1007/BF01894026.

Kanervo V. 1942. Schadeninsekten in Ostkarelien im Sommer 1942. Annales entomologici Fennici 8: 260.

Kanmiya K. 1983. A systematic study of the Japanese Chloropidae (Diptera). Memoirs of the Entomological Society of Washington 11: $1-370$.

Karpa A. 2001. Revision of Chloropidae of the collection of B.A. Gimmerthal and a check-list of Latvian Chloropidae (Diptera). Latvijas Entomologs 38: 21-26.

Karpova AI. 1972. Ecological and geographic description of the field fauna of grass flies (Diptera, Chloropidae). Entomological Review 51(4): 484-491.

Khaghaninia S, Gharajedaghi Y. 2013. A study of the family Chloropidae (Diptera) in grasslands of East Azerbaijan province. Iranian Journal of Forest and Range Protection Research 11(1): 68-77. doi: 10.22092/ IJFRPR.2013.106401.

Kiauka GF, Nartshuk EP. 1973. On biology of Polyodaspis rufijicornis Mcq. (Diptera, Chloropidae). Entomological Review 52: 434-438.

Kotrba M, Nartshuk E. 2008. Masseauftreten der Gemeinen Rasenhalmfliege Thaumatomyia notata (Diptera: Chloropidae) an Gebäuden: Determination-Biologie-Verbreitung. Studia dipterologica 15(1/2): 193-209.

Kozlov MV, Zvereva EL. 1997. Effects of pollution and urbanization on diversity of frit flies (Diptera, Chloropidae). Acta oecologica 18: 13-20. doi: 10.1016/S1146-609X(97)80076-8.

Kravchenko AV. 2007. A compendium of Karelian flora (vascular plants). Petrozavodsk. Karelian Research Centre RAS. 403 p. (In Russian).

Krogerus R. 1960. Ökologische Studien über nordische Moorarthropoden. Commentationes biologicae 21(3): 1-238.

Kubik S, Barták M. 2014. The preliminary result of the frit flies research (Diptera: Chloropidae) in Mugla province (SW Turkey). In: Dorchin N, Kotrba M, Mengual X, Menzel F. (eds). 8th International Congress of Dipterology, 10-15 August 2014, Potsdam, Germany. Abstract Volume. Halle (Saale). Ampyx-Verlag Dr. Andreas Stark. pp 188.

Merz B. 2012. 8.36.31. Superfamily Carnoidea. In: Merz B. (ed). Liste annotée des insectes (Insecta) du canton de Genève. Instrumenta Biodiversitatis VIII. Genève. Muséum d'histoire naturelle. pp 404-407.

Nartshuk EP. 1962. On the fauna and ecology of Chloropidae (Diptera) of the Leningrad Province. Trudy Zoologicheskogo Instituta Akademii Nauk SSSR 31: 250-275. (In Russian).

Nartshuk EP. 1984. Family Chloropidae. In: Soós Á, Papp L. (eds). Catalogue of Palaearctic Diptera. Vol. 10 (Clusiidae-Chloropidae). Budapest. Akadémiai Kiadó. pp 232-298.

Nartshuk EP. 1992. Revision of the species of Meromyza Mg. from Finland. Entomologica Fennica 3(3): 121-138. doi: 10.33338/ef.83603.

Nartshuk EP. 1998. A revision of grassflies of the tribe Chloropini (Diptera, Chloropidae) of Finland, Estonia and North-West Russia. Entomologica Fennica 9: 153-183. doi: 10.33338/ef.83979.

Nartshuk EP. 1999a. Grassflies of the subfamily Chloropinae, except the tribe Chloropini and the genus Meromyza, of Finland, Karelia and the Kola Peninsula (Diptera, Cyclorrhapha, Chloropidae). Entomologica Fennica 10: 7-28. doi: 10.33338/ef.83994.

Nartshuk EP. 1999b. Notes on Cetema elongatum species group (Diptera, Chloropidae). International Journal of Dipterological Research 10(1): $67-70$.

Nartshuk EP. 1999c. The first record of carnivorous chloropid fly Pseudogaurax venustus (Czerny, 1906) (Diptera, Chloropidae) in Russia. International Journal of Dipterological Research 10(2): 77-79. 
Nartshuk E. 2000. Outbreaks of carnivorous fly Thaumatomyia notata Meigen (Diptera, Chloropidae) and their periodicity. Proceedings of the Zoological Institute RAS. 286: 93-100.

Nartshuk EP. 2005. Grassflies (Diptera, Chloropidae) in the Northern Palaearctic Region. Entomological Review 85(3): 244-254.

Nartshuk EP. 2006. A revision of Meigen's Chloropidae collection in the Museum national d'Histoire naturelle, Paris (Diptera). Zoosystematica Rossica 15(1): 173-184.

Nartshuk EP. 2008. Gaurax flavomaculatus (Duda, 1933) (Diptera, Chloropidae) found in Norway - with data on distribution and the mode of life of the species. Norwegian Journal of Entomology 55(2): 233-234.

Nartshuk EP. 2014. Grass-fly larvae (Diptera, Chloropidae): diversity, habitats, and feeding specialization. Entomological Review 94(4): 514-525. doi: 10.1134/S001387381404006X.

Nartshuk EP. 2015. Niche separation between two closely related species of the genus Lasiambia Anonymous, 1937 (Diptera: Chloropidae). Caucasian Entomological Bulletin 11(2): 415-417. doi: 10.23885/18143326-2015-11-2-415-417.

Nartshuk EP, Andersson H. 2013. The frit flies (Chloropidae, Diptera) of Fennoscandia and Denmark. In: Kristensen NP, Michelsen V. (eds). Fauna entomologica Scandinavica, 43. Leiden-Boston. Brill. pp 1-282. doi: $10.1163 / 9789004190665002$.

Nartshuk E, Elberg K. 1979. The list of frit flies (Diptera, Chloropidae) of Estonia. In: Elberg K. (ed). Dipteroloogilisi uurimusi [Dipterological Research]. Eesti NSV Teaduste Akadeemia Toimetus-ja Kirjastus Nõukogu. Tallinn. pp 122-143. (In Russian).

Nartshuk EP, KahanpääJ. 2014. Checklist of the family Chloropidae (Diptera) of Finland. Zookeys 441: 311-318. doi: 10.3897/zookeys.441.7505.

Nartshuk EP, Przhiboro AA. 2003. New records of Chloropidae from Russia and adjacent countries (Diptera). Zoosystematica Rossica 11(2): 350 .

Nartshuk EP, Przhiboro AA. 2009. A new species of Incertella Sabrosky (Diptera: Chloropidae) from the White Sea coast, Russian Karelia. Entomologica Fennica 20(1): 4-8. doi: 10.33338/ef.84453.

Nielsen LB. 2014. Distribution of Oscinellinae (Diptera, Chloropidae) in the Danish landscape. Entomologiske Meddelelser 82(1): 39-62.

Nielsen LB. 2015. Distribution of Chloropinae (Diptera, Chloropidae) in the Danish landscape. Entomologiske Meddelelser 83(1): 3-20.

Nielsen NO, Nielsen LB, Toft S. 2019. Epigaeic Diptera Brachycera from the coastal sand dunes of National Park Thy, Denmark. Entomologiske Meddelelser 87(1-2): 19-40.

Nye IWB. 1958. The external morphology of some of the dipterous larvae living in the Gramineae of Britain. Transactions of the Royal Entomological Society of London 110(15): 411-487. doi: 10.1111/j.13652311.1958.tb00380.x.

Nye IWB. 1959. The distribution of shoot-fly larvae (Diptera, Acalypterae) within pasture grasses and cereals in England. Bulletin of Entomological Research 50(1): 53-62. doi: 10.1017/S0007485300054419.

Pakalniškis $\mathrm{S}$, Bernotiene R, Lutovinovas E, Petrašiūnas A, Podènas S, Rimšaite J, Sæther OA, Spungis V. 2006. Checklist of Lithuanian Diptera. New and rare for Lithuania insect species 18: 16-154.

Petersen FT. 2001. A preliminary list of the Diptera of Denmark. Chloropidae. Steenstrupia 26(2): 189-191.

Polevoi AV. 1997. On the fauna of some brachycerous Diptera (Diptera, Brachycera Orthorrhapha, Brachycera Cyclorrhapha) of Kivach Nature Reserve. In: Kravchenko AV. (ed). Flora and fauna of nature reserves. Petrozavodsk. Karelian Research Centre RAS. pp 30-43. (In Russian).

Polevoi AV. 2006. New data on the Diptera fauna of Kivach Nature Reserve. Transactions of Karelian Research Centre of the Russian Academy of Sciences 10: 94-105. (In Russian).

Polevoi AV, Humala AE. 2003. Insects. In: Gromtsev AN. (ed). Data on inventory of natural complexes and scientific feasibility study of the "Syrovatka" landscape reserve. Petrozavodsk. Karelian Research Centre RAS. pp 67-72. (In Russian).

Polevoi AV, Humala AE. 2005. Insects. In: Gromtsev AN. (ed). Nature complexes of the Vepsian Volost: featires, present-day status, conservation and management. Petrozavodsk. Karelian Research Centre RAS. pp 172-186. (In Russian).

Polevoi AV, Humala AE. 2007. Insects. In: Gromtsev AN. (ed). Inventory materials on the natural environment and nature assessment of the "Chukozero" area. Petrozavodsk. Karelian Research Centre RAS. pp 85-89. (In Russian).

Polevoi AV, Humala AE. 2009. Insects. In: Gromtsev AN. (ed). Nature of Mount Vottovaara: characteristic, condition, conservation. Petrozavodsk. Karelian Research Centre RAS. pp 106-118. (In Russian).

Pschorn-Walcher H. 1956. Chloropiden als Kostgänger bei Chrysomelidenlarven. Zoologischer Anzeiger 156(11-12): 318-319.

Rollard C. 1987. La biocenose associée aux araneides, en landes armoricaines. Étude des relations insectes - araignées. Thèse de Doctorat de l'Université de Rennes. 293 p.

Rollard 1992. Révision des Insectes consommateurs d'œfs d‘Araignées. II. Les Diptères et les Névroptères. Bulletin de la Socièté entomologique de Mulhouse 1992: 41-47.

Ryu HS. 1994. Check list of insects from Korea. Entomological Society of Korea and Korean Society of Applied Entomology. 745 p.

Sabrosky CW. 1967. Corrections to a catalog of the Diptera of America north of Mexico. Bulletin of the Entomological Society of America 13(2): 115-125. doi: 10.1093/besa/13.2.115.

Savage J, Wheeler TA. 1999. Systematics of Cetema Hendel (Diptera: Chloropidae): revision of the Nearctic species and phylogeny and zoogeography of the Holarctic fauna. Insect Systematics \& Evolution 30(3): 249-262. doi: 10.1163/187631200X00101.

Stackelberg AA. 1958. On the Diptera fauna of the Leningrad Province. III. Diptera. Acalyptrata. Part I. Trudy Zoologicheskogo Instituta Akademii Nauk SSSR 24: 103-191. (In Russian).

von Tschirnhaus M. 1981. Die Halm- und Minierfliegen im Grenzbereich Land-Meer der Nordsee. Eine Oekologische Studie mit Beschreibung von zwei neuen Arten und neuen Fang- und Konservierungsmethoden (Diptera: Chloropidae et Agromyzidae). Spixiana, Zeitschrift für Zoologie, Supplement 6: 1-405.

von Tschirnhaus M. 1992. Minier- und Halmfliegen (Agromyzidae, Chloropidae) und 52 weitere Familien (Diptera) aus Malaise-Fallen in Kiesgruben und einem Vorstadtgarten in Köln. Decheniana, Beihefte 31: 445-497.

von Tschirnhaus M, Borkenstein A, Jödicke R. 2015. Lestes dryas (Odonata: Lestidae) und kommensalische Fliegen (Diptera: Chloropidae), mit einer Übersicht über Kleptoparasitismus bei Halmfliegen. Mercuriale 14(2014): 1-12.

Wendt H. 1999. Checkliste der Dipteren Deutschlands. Chloropidae. Studia dipterologica, Supplement 2: 140-143.

Yakovlev E, Scherbakov A, Polevoi A, Humala A. 2000. Insect fauna of Paanajärvi National Park and proposed Kalevala National Park with particular emphasis on saproxylic Coleoptera, Diptera and Hymenoptera. In: Heikkilä R, Heikkilä M, Polevoi A, Yakovlev E. (eds). Biodiversity of old-growth forests and its conservation in the northwestern Russia. Oulu. North Ostrobothnia Regional Environmental Centre. pp 103-157.

Zatwarnicki T. 2001. Wykaz Muchówek Polski. Check-list of Polish Diptera. Wersja: IV 2001. <http://www.online-keys.net/sciaroidea/2000_/ Muchowki_2001_Checl_list_of_polish_diptera.pdf $>$. Accessed: 2020.04.20.

Znamenskaya MK. 1941. Pests of cereals and fodder grasses in Khibines. Vestnik Zashchity Rastenii 1941(1): 42-44. (In Russian).

Znamenskaya MK. 1962. A review of the crop pests of the Murmansk region. Entomological Review 41(2): 190-194.

Editorial responsibility: Torkild Bakken.

This article is open-access and distributed under the terms of the Creative Commons Attribution 4.0 International license. This permits all noncommercial use, distribution, and reproduction in any medium, provided the original work is properly cited.

(http://creativecommons.org/licenses/by/4.0/). 
Appendix I. Collecting localities of Chloropidae in the Russian part of Fennoscandia. See Figure 1 for abbreviations of provinces.

\begin{tabular}{|c|c|c|c|c|}
\hline Province & Locality name & Outdated and label names & Position (WGS84) & Comment \\
\hline \multicolumn{5}{|c|}{ Murmansk province } \\
\hline Lps & Borisoglebskii & Kolttaköngäs & N69.654:E30.134 & \\
\hline Lps & Korablekk Mt. & & N69.239:E29.468 & \\
\hline Lps & Kuvernöörikoski & Kuvernööri & N69.510:E30.423 & \\
\hline Lps & Langvatn & & N69.365:E29.746 & $\begin{array}{l}\text { Expansion (lake) of the Paz (Paatsjoki) River } \\
\text { (Pasvik Nature Reserve) }\end{array}$ \\
\hline Lps & Lotta River & Fl. Lutto, Lutto & $\begin{array}{l}\text { N68.475:E28.512 } \\
\text { N68.576:E29.408 }\end{array}$ & $\begin{array}{l}\text { B. Poppius and S. Platonoff collected in several } \\
\text { localities along the river (all in Lps). We give rough } \\
\text { coordinates approximately corresponding to the } \\
\text { middle point of each collector's route. }\end{array}$ \\
\hline Lps & Niilansaari Isl. & Varlama Isl., Varlamsaari & N69.141:E29.241 & \\
\hline Lps & Pechenga & Petsamo & N69.547:E31.209 & \\
\hline Lps & Salmijärvi & & N69.437:E30.118 & \\
\hline Lps & Trifonovo & Trifona & N69.595:E31.266 & \\
\hline Lps & Vuoremijärvi & Vuoremi & N69.553:E30.916 & \\
\hline Lps & Yläluostari & & N69.423:E31.049 & \\
\hline Lps & Zemlyanoe & Pummanki & N69.786:E31.961 & \\
\hline Lt & Kola & & N68.881:E33.015 & \\
\hline$L t$ & Murmansk & & N68.968:E33.078 & \\
\hline$L t$ & Notozero & Nuortijärvi, Lac. Nuorti & N68.429:E30.994 & Nowadays flooded by Verkhnetulomskoe dam lake \\
\hline Lt & Polyarnyi & Aleksandrovsk & N69.199:E33.444 & \\
\hline Lt & Tuloma River & & N68.881:E33.006 & Location inside modern limits of the Kola township \\
\hline$L t$ & Tyuva-Guba & & N69.185:E33.635 & \\
\hline$L t$ & $\begin{array}{l}\text { Verhnetulomskii } \\
\text { (50 km W of })\end{array}$ & & $\mathrm{N} 68.595: \mathrm{E} 30.526$ & \\
\hline Lmur & Dal'nie Zelentsy & & N69.114:E36.063 & \\
\hline Lmur & Gavrilovo & Gavrilova & N69.177:E35.845 & \\
\hline Lmur & Lovozero & & N67.998: E35.024 & \\
\hline Lmur & Mishukovo & & N69.045:E33.037 & \\
\hline Lmur & Seidozero Lake & Seidjaur & N67.825:E34.853 & \\
\hline Lmur & Tumannyi & & N68.883:E35.692 & \\
\hline Lmur & Voron'ya River & Fl. Voron & N68.860:E35.601 & $12-50 \mathrm{~km}$ from the mouth of river \\
\hline Lmur & Voroninsk & $\begin{array}{l}\text { Pg. Voron, Voroninskii } \\
\text { pogost }\end{array}$ & N68.455:E35.344 & Nowadays flooded by Serebryanskoe dam lake \\
\hline Lim & Apatity & & N67.563:E33.367 & \\
\hline Lim & Belaya Guba & Bjäloguba & N67.675:E33.235 & $\begin{array}{l}\text { Bay of Imandra Lake near Apatity. Materials with } \\
\text { this label were collected along the Malaya Belaya } \\
\text { (Lutarmajok) River }\end{array}$ \\
\hline Lim & Berezhnoi Vlasov Isl. & & N67.084:E32.689 & \\
\hline Lim & Bolshoi Vudjavr Lake & & N67.636:E33.652 & \\
\hline Lim & Kandalaksha & $\begin{array}{l}\text { Kantalaks, Kantalahti, } \\
\text { Kandalahti }\end{array}$ & N67.155:E32.412 & \\
\hline Lim & Khibiny & Chibinä & N67.674:E33.210 & \\
\hline Lim & Kirovsk & & N67.616:E 33.667 & \\
\hline Lim & Kokorin Stream & & N67.635:E32.687 & Small river in Laplandskii Nature Reserve \\
\hline Lim & Kolvitsa & & N67.086:E32.983 & \\
\hline Lim & Lisii Stream & & $\begin{array}{l}\text { N67.653:E32.595 } \\
\text { N67.651:E32.599 }\end{array}$ & Small river in Laplandskii Nature Reserve \\
\hline
\end{tabular}


Appendix I. Continued.

\begin{tabular}{|c|c|c|c|c|}
\hline Province & Locality name & Outdated and label names & Position (WGS84) & Comment \\
\hline Lim & Luven'ga & & $\begin{array}{l}\text { N67.105:E32.697 } \\
\text { N67.102:E32.713 } \\
\text { N67.097:E32.709 }\end{array}$ & \\
\hline Lim & Malyi Vudjavr Lake & & N67.667:E33.619 & \\
\hline Lim & Monchegorsk & & N67.938:E32.935 & \\
\hline Lim & Ryashkov Isl. & & N67.017:E32.557 & \\
\hline Lim & Telyachii Isl. & & N67.115:E32.315 & \\
\hline Lim & Umba & & N66.679:E34.344 & \\
\hline Lim & Vtoroi Stream & & N67.654:E32.637 & Small river in Laplandskii Nature Reserve \\
\hline Lim & Yuksporrjok River & & N67.638:E33.718 & \\
\hline$L v$ & Varzuga & & N66.407:E36.584 & \\
\hline$L p$ & Ponoi & Ponoj & N67.076:E41.126 & \\
\hline Ks & Kuolajärvi & Kuolajärvi (Salla) & N66.975:E29.252 & \\
\hline$K k$ & Kovda & Kouta & N66.691:E32.868 & Village south-east of Zelenoborskii \\
\hline \multicolumn{5}{|l|}{ Karelia } \\
\hline Ks & Leppälä & & N66.272:E30.088 & Abandoned village in the Paanajärvi area \\
\hline$K s$ & Nuorunen Mt. & & N66.144:E30.235 & \\
\hline$K s$ & Paanajärvi & & N66.262:E29.812 & $\begin{array}{l}\text { Average coordinates for Paanajärvi area to allow } \\
\text { mapping of the published data }\end{array}$ \\
\hline Ks & Vartolambina & Vartiolampi & N66.242:E30.576 & Abandoned village in the Paanajärvi area \\
\hline Kk & Chupa & & N66.275:E33.056 & \\
\hline Kk & Gridino & & $\begin{array}{l}\text { N65.982:E34.696 } \\
\text { N65.966:E34.716 } \\
\text { N65.924:E34.688 } \\
\text { N65.918:E34.661 } \\
\text { N65.906:E34.599 } \\
\text { N65.869:E34.605 }\end{array}$ & \\
\hline Kk & Kartesh & & $\begin{array}{l}\text { N66.366:E33.735 } \\
\text { N66.365:E33.603 } \\
\text { N66.350:E33.592 } \\
\text { N66.348:E33.616 } \\
\text { N66.346:E33.623 } \\
\text { N66.344:E33.621 } \\
\text { N66.341:E33.669 } \\
\text { N66.339:E33.635 } \\
\text { N66.339:E33.659 } \\
\text { N66.337:E33.649 } \\
\text { N66.335:E33.615 }\end{array}$ & $\begin{array}{l}\text { Localities in the vicinity of the White Sea } \\
\text { Biological Station of the Zoological Institute of } \\
\text { Russian Academy of Sciences (St Petersburg) }\end{array}$ \\
\hline$K k$ & Keret' & & N66.273:E33.552 & \\
\hline Kk & Kuzema & & N65.358:E34.253 & \\
\hline Kk & Malyi Andronin Isl. & & N66.334:E33.763 & \\
\hline Kk & Nikol'skaya Bay & & N66.206:E33.912 & \\
\hline$K k$ & Pezhostrov Isl. & & N66.258:E33.887 & \\
\hline Kk & Primorskii & & $\begin{array}{l}\text { N66.553:E33.093 } \\
\text { N66.552:E33.102 } \\
\text { N66.552:E33.114 }\end{array}$ & $\begin{array}{l}\text { The White Sea Biological Station of Moscow State } \\
\text { University and localities on the mainland nearby }\end{array}$ \\
\hline Kk & Sidorov Isl. & & N66.348:E33.823 & \\
\hline Kk & Sonostrov & Sonostroff & $\begin{array}{l}\text { N66.158:E34.223 } \\
\text { N66.170:E34.222 }\end{array}$ & Village and neighboring island \\
\hline Kk & Syrovatka & & $\begin{array}{l}\text { N65.552:E34.736 } \\
\text { N65.539:E34.711 } \\
\text { N65.528:E34.730 } \\
\text { N65.515:E34.688 } \\
\text { N65.512:E34.738 }\end{array}$ & $\begin{array}{l}\text { Small island in the White Sea north of Kuzema and } \\
\text { localities on the mainland nearby }\end{array}$ \\
\hline
\end{tabular}


Appendix I. Continued.

\begin{tabular}{|c|c|c|c|c|}
\hline Province & Locality name & Outdated and label names & Position (WGS84) & Comment \\
\hline Kрос & Belomorsk & Soroka & $\begin{array}{l}\text { N64.544:E34.775 } \\
\text { N64.529:E34.779 }\end{array}$ & \\
\hline Kpoc & Kem' & & N64.958:E34.603 & \\
\hline Kpoc & Kostomuksha & Kostamus & N64.588:E30.595 & \\
\hline Kpoc & Kuzharvi Lake & & N63.615:E32.559 & \\
\hline Kpoc & Ladvozero & Latvajärvi & $\begin{array}{l}\text { N64.829:E29.882 } \\
\text { N64.890:E29.830 }\end{array}$ & Localities near the lake and homonymic village \\
\hline Kрос & Levi Lake & & N65.142:E29.948 & \\
\hline Kpoc & Lodeinyi Isl. & & N64.918:E35.165 & \\
\hline Kpoc & Murdoioki River & & N64.202:E30.866 & \\
\hline Kpoc & Nemetskii Kuzov Isl. & & N64.952:E35.162 & \\
\hline Kpoc & Nesterova Mt. & & N63.713:E32.505 & \\
\hline Kpoc & Russkii Kuzov Isl. & & N64.935:E35.128 & \\
\hline Kpoc & Shueretskoe & Tschuja & N64.751:E34.724 & \\
\hline Kpoc & Taparukha Isl. & & N64.986:E35.028 & \\
\hline Kpoc & Voknavolok & Vuokiniemi & N64.952:E30.542 & \\
\hline Kpoc & Zhiloi Isl. & & N64.945:E35.239 & \\
\hline Kpor & Bolshoi Zhuzhmui Isl. & & N64.677:E35.560 & \\
\hline Kpor & Kondostrov Isl. & & N64.224:E36.622 & \\
\hline Kpor & Ladozero Lake & & N63.563:E35.691 & \\
\hline Kpor & Malyi Zhuzhmui Isl. & & N64.619:E35.669 & \\
\hline Kpor & Myagostrov Isl. & & N64.372:E35.960 & \\
\hline Kpor & Nadvoitsy & Wojatsch & N63.880:E34.261 & \\
\hline Kpor & Pechak Isl. & & N64.626:E35.629 & \\
\hline Kpor & $\begin{array}{l}\text { Perkhludy Archipelago. } \\
\text { Yuzhnyi Isl. }\end{array}$ & & N64.324:E36.479 & \\
\hline Kpor & Segezha & Sekehen, Säkehä & $\begin{array}{l}\text { N63.744:E34.299 } \\
\text { N63.727:E34.249 }\end{array}$ & \\
\hline Kpor & Sumskii Posad & & N64.255:E 35.410 & \\
\hline Kpor & Valdai & & N63.547:E35.563 & \\
\hline$K b$ & Tolvojärvi & & N62.317:E31.435 & \\
\hline Kon & Ar'koila & & N61.935:N32.842 & \\
\hline Kon & Belaya Gora & & N62.582:E33.959 & \\
\hline Kon & Bolshoi Lelikovskii Isl. & & N61.935:E35.141 & \\
\hline Kon & Bukol'nikov Isl. & & N62.005:E35.199 & \\
\hline Kon & Eglov Isl. & & N62.129:E35.170 & \\
\hline Kon & Ernitskii Isl. & & N61.979:E35.176 & \\
\hline Kon & Girvas & & $\begin{array}{l}\text { N62.501:E33.681 } \\
\text { N62.456:E33.687 }\end{array}$ & \\
\hline Kon & Gomsel'ga & & N62.060:E33.992 & \\
\hline Kon & Karel'skii Isl. & & N62.014:E35.209 & \\
\hline Kon & Khvost Isl. & & N62.149:E35.150 & \\
\hline Kon & Kivach & & $\begin{array}{l}\text { N62.281:E33.967 } \\
\text { N62.272:E33.986 } \\
\text { N62.272:E33.986 }\end{array}$ & $\begin{array}{l}\text { Three localities in the central part of Kivach Nature } \\
\text { Reserve }\end{array}$ \\
\hline Kon & Kizhi Isl. & & N62.082:E35.224 & \\
\hline Kon & Klimenitsy & & $\begin{array}{l}\text { N61.901:E35.216 } \\
\text { N61.890:E35.199 }\end{array}$ & $\begin{array}{l}\text { Localities north of abandoned village in the south } \\
\text { part of Bolshoi Klimenetskii Island }\end{array}$ \\
\hline
\end{tabular}


Appendix I. Continued.

\begin{tabular}{|c|c|c|c|c|}
\hline Province & Locality name & Outdated and label names & Position (WGS84) & Comment \\
\hline Kon & Konchezero & Kenjärvi & $\begin{array}{l}\text { N62.154:E34.013 } \\
\text { N62.129:E34.013 } \\
\text { N62.107:E33.993 } \\
\text { N62.084:E34.037 }\end{array}$ & \\
\hline Kon & Kondopoga & Kontupohja & N62.206:E34.266 & \\
\hline Kon & Kosalma & & N62.034:E34.102 & \\
\hline Kon & Kuivakhda Isl. & & N61.965:E35.168 & \\
\hline Kon & Kurgenitsy & & N62.083:E35.321 & \\
\hline Kon & Lambaznik Isl. & & N61.995:E35.190 & \\
\hline Kon & Lelikovo & & $\begin{array}{l}\text { N61.991:E35.148 } \\
\text { N61.978:E35.160 }\end{array}$ & $\begin{array}{l}\text { Two localities near the village on Malyi Lelikovskii } \\
\text { Island }\end{array}$ \\
\hline Kon & Lipovitsy & & N62.132:E35.093 & \\
\hline Kon & Lisitsyno & & N62.356:E35.539 & \\
\hline Kon & Lizhma & & N62.420:E34.450 & \\
\hline Kon & Lyudskoi Isl. & & $\begin{array}{l}\text { N61.970:E35.175 } \\
\text { N61.963:E35.179 }\end{array}$ & \\
\hline Kon & Martsial'nye Vody & Dvortsy, Dvoretz & N62.156:E33.875 & \\
\hline Kon & Medvezh'egorsk & Karhumäki & $\begin{array}{l}\text { N62.911:E34.444 } \\
\text { N62.893:E34.365 }\end{array}$ & \\
\hline Kon & Myagrozero & & N62.470:E34.815 & \\
\hline Kon & Myal’ Isl. & & N61.999:E35.148 & \\
\hline Kon & Nizhnee Myagrozero & & N62.507:E34.782 & \\
\hline Kon & Nizhnii Myarat Lake & & N63.113:E33.058 & \\
\hline Kon & Oyatevschina & & N62.076:E35.139 & \\
\hline Kon & Padany & Paadana & N63.286:E33.419 & \\
\hline Kon & Paleostrov Isl. & & N62.569:E35.259 & \\
\hline Kon & Pin'guba & & N61.865:E34.556 & $\begin{array}{l}\text { Bay of Onego Lake and a cluster of summer cot- } \\
\text { tages }\end{array}$ \\
\hline Kon & Pod'el'niki & & $\begin{array}{l}\text { N62.106:E35.174 } \\
\text { N62.138:E35.130 }\end{array}$ & \\
\hline Kon & Polya & & N62.290:E35.309 & \\
\hline Kon & Radkol'e Isl. & & N61.996:E35.186 & \\
\hline Kon & Raiguba & & N62.371:E33.770 & \\
\hline Kon & Semchezero & Semsjärvi & N62.949:E33.608 & \\
\hline Kon & Shaidoma & & $\begin{array}{l}\text { N62.809:E34.192 } \\
\text { N62.789:E34.138 } \\
\text { N62.757:E34.181 } \\
\text { N62.751:E34.107 } \\
\text { N62.750:E34.150 } \\
\text { N62.734:E34.201 }\end{array}$ & Six localities in Shaidomskii Reserve \\
\hline Kon & Shuyal Lake & & N63.221:E32.888 & \\
\hline Kon & Solomennoe & Solomina & N61.847:E34.346 & \\
\hline Kon & Sychevets Isl. & & N62.029:E35.201 & \\
\hline Kon & Tereki & & N62.213:E33.875 & \\
\hline Kon & Tipinitsy & & N62.158:E35.462 & \\
\hline Kon & Tivdiya & Tiutia, Tivdia, Tiudie, Tjudi & $\mathrm{N} 62.562: \mathrm{E} 33.956$ & \\
\hline Kon & Vegoruksy & & N62.221:E34.878 & \\
\hline Kon & Velikaya Niva & & N62.359:E35.219 & \\
\hline Kon & Vendery & & $\begin{array}{l}\text { N62.241:E33.297 } \\
\text { N62.249:E33.296 }\end{array}$ & \\
\hline
\end{tabular}


Appendix I. Continued.

\begin{tabular}{|c|c|c|c|c|}
\hline Province & Locality name & Outdated and label names & Position (WGS84) & Comment \\
\hline Kon & Vikshezero Lake & & $\begin{array}{l}\text { N62.582:E34.372 } \\
\text { N62.568:E34.353 }\end{array}$ & \\
\hline Kon & Vorob'i & & $\begin{array}{l}\text { N62.063:E35.256 } \\
\text { N62.046:E35.249 }\end{array}$ & \\
\hline Kon & Vottovaara Mt. & & N63.084:E32.625 & \\
\hline Kon & Yalguba & Jalguba & N61.886:E34.567 & \\
\hline Kton & Besov Nos & & N61.672:E36.029 & \\
\hline Kton & Kolgostrov & & N62.380:E36.935 & $\begin{array}{l}\text { Island and abandoned village (Vodlozerskii } \\
\text { National Park) }\end{array}$ \\
\hline Kton & Kolovo & & N61.814:E36.674 & \\
\hline Kton & Koskosalma & & N62.401:E36.997 & $\begin{array}{l}\text { Abandoned village and field base (Vodlozerskii } \\
\text { National Park) }\end{array}$ \\
\hline Kton & Novguda River & & N62.542:E37.007 & $\begin{array}{l}\text { Field base in the river mouth (Vodlozerskii National } \\
\text { Park) }\end{array}$ \\
\hline Kton & Sheltoporog & & N63.081:E35.632 & \\
\hline Kton & Shoikapolda River & & N62.528:E37.377 & \\
\hline Kton & Sukhaya Vodla River & & $\begin{array}{l}\text { N62.413:E37.110 } \\
\text { N62.396:E37.360 }\end{array}$ & $\begin{array}{l}\text { Localities along the upper course of the river (partly } \\
\text { in Vodlozerskii National Park) }\end{array}$ \\
\hline Kton & Vozritsy & & N62.659:E35.391 & \\
\hline$K l$ & Haapalampi & & N61.646:E30.581 & \\
\hline$K l$ & Harlu & & N61.805:E30.934 & \\
\hline$K l$ & Impilahti & Impilaks & N61.675:E31.158 & \\
\hline$K l$ & Iso-Iijärvi Lake & & N61.597:E29.921 & \\
\hline$K l$ & Jaakkima & & N61.512:E30.129 & \\
\hline$K l$ & Kilpola Isl. & & $\begin{array}{l}\text { N61.203:E29.960 } \\
\text { N61.203:E30.003 } \\
\text { N61.182:E29.968 }\end{array}$ & \\
\hline$K l$ & Kirjävalahti & Kirjävalaks & N61.781:E30.782 & \\
\hline$K l$ & Kurkijoki & & N61.300:E29.874 & \\
\hline$K l$ & Meijeri & & $\begin{array}{l}\text { N61.627:E30.552 } \\
\text { N61.620:E30.588 }\end{array}$ & \\
\hline$K l$ & Niemelänhovi & & N61.608:E30.587 & \\
\hline$K l$ & Niva & & $\begin{array}{l}\text { N61.634:E30.252 } \\
\text { N61.615:E30.274 }\end{array}$ & \\
\hline$K l$ & Puikkola & & $\begin{array}{l}\text { N62.051:E30.662 } \\
\text { N62.046:E30.857 }\end{array}$ & \\
\hline$K l$ & Reuskula & & $\begin{array}{l}\text { N61.621:E30.393 } \\
\text { N61.602:E30.443 }\end{array}$ & \\
\hline$K l$ & Ruskeala & & N61.928:E30.576 & \\
\hline$K l$ & Salmi & & N61.366:E31.857 & \\
\hline$K l$ & Sortavala & Sordavala & N61.693:E30.670 & \\
\hline$K l$ & Suistamo & & N61.917:E31.143 & \\
\hline$K l$ & Sukopohja & & N61.687:E30.160 & \\
\hline$K l$ & Valaam & Valamo, Walamo & $\begin{array}{l}\text { N61.393:E30.910 } \\
\text { N61.389:E30.947 } \\
\text { N61.371:E30.958 } \\
\text { N61.362:E30.986 } \\
\text { N61.350:E30.990 }\end{array}$ & Localities in different parts of the archipelago \\
\hline Kol & Bol'shie Gory & Suurimäki & N61.312:E32.463 & \\
\hline Kol & Derevyannoe & Puujoki & N61.615:E34.640 & \\
\hline Kol & Gizhino & Kuujärvi & N60.994:E33.788 & \\
\hline
\end{tabular}


Appendix I. Continued.

\begin{tabular}{|c|c|c|c|c|}
\hline Province & Locality name & Outdated and label names & Position (WGS84) & Comment \\
\hline Kol & Kaskesruchei & & $\begin{array}{l}\text { N61.238:E35.591 } \\
\text { N61.203:E35.594 } \\
\text { N61.194:E35.585 }\end{array}$ & \\
\hline
\end{tabular}

Kol Kindasovo

Kol Kolatsel'ga

Kol Lososinnoe

Kol Matveeva Sel'ga

Kol Mayachino

Kol Novikovo

Kol Olonets

Kol Pai

Kol Petrozavodsk

Kol Sändeba

Kol Sheltozero

Kolatselkä

N61.725:E33.468

N61.685:E32.231

N61.708:E34.241

N61.295:E35.176

N60.777:E32.818

N60.757:E32.816

N61.070:E33.777

N60.980:E32.958

N61.210:E34.423

Äänislinna. Petrosawodsk

Säntämä

Soutjärvi

N61.163:E32.765

N61.401:E35.335
N61.775:E34.341

N61.393:E35.308

N61.370:E35.358

N61.364:E35.422

$\begin{array}{ll}\text { Kol } & \text { Shoksha } \\ \text { Kol } & \text { Sudalitsa } \\ \text { Kol } & \text { Ust'e Obzhank } \\ \text { Kol } & \text { Ust'e Tuloksy }\end{array}$

N61.473:E35.141

N60.964:E32.974

N60.822:E32.819

N61.129:E32.524

N61.113:E32.552

$\begin{array}{lll}\text { Kol } & \text { Vedlozero } & \text { Vielijärvi } \\ \text { Kol } & \text { Vidlitsa } & \text { Viitele }\end{array}$

Jalolahti

N61.579:E32.832

N61.190:E32.393

N61.193:E32.314

N61.179:E32.429

N61.177:E32.370

N61.156:E32.457

N61.331:E35.369

N61.886:E34.567

N61.908:E37.767

N61.793:E37.511

N61.708:E37.705

\section{Arkhangelsk province}

Kton Vozhosel'ga

N62.774:E37.159

Locality in the mouth of Vyzhiga River

(Vodlozerskii National Park)

\section{Leningrad province}

Kol Gimreka

N61.153:E35.618

N61.151:E35.640

$\begin{array}{llll}\text { Kol } & \text { Gumbaritsy } & \text { Gumbaritsa } & \text { N60.684:E32.931 } \\ \text { Kol } & \text { Lahta } & \text { Kutlahta } & \text { N60.643:E33.086 } \\ \text { Kol } & \text { Moshnich'e } & & \text { N61.149:E33.854 } \\ \text { Kol } & \text { Svir' River } & \text { Fl. Swir } & \text { N60.946:E34.096 } \\ \text { Kol } & \text { Vazhiny } & \text { Vaaseni, Vaašeni } & \text { N60.963:E34.024 }\end{array}$


Appendix 2. Distribution maps of Chloropidae species, occurring in the Russian part of Fennoscandia.
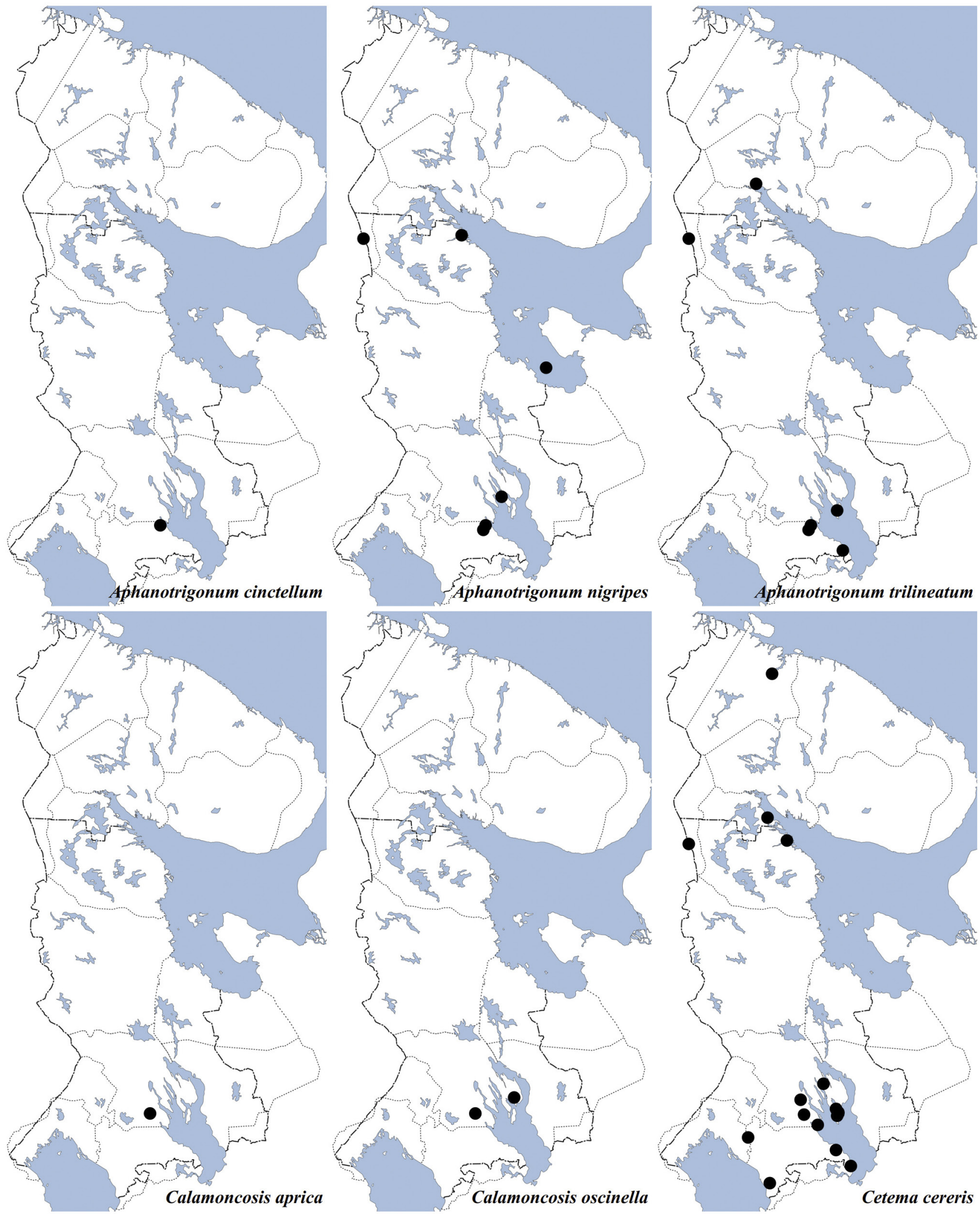
Appendix 2. Continued.
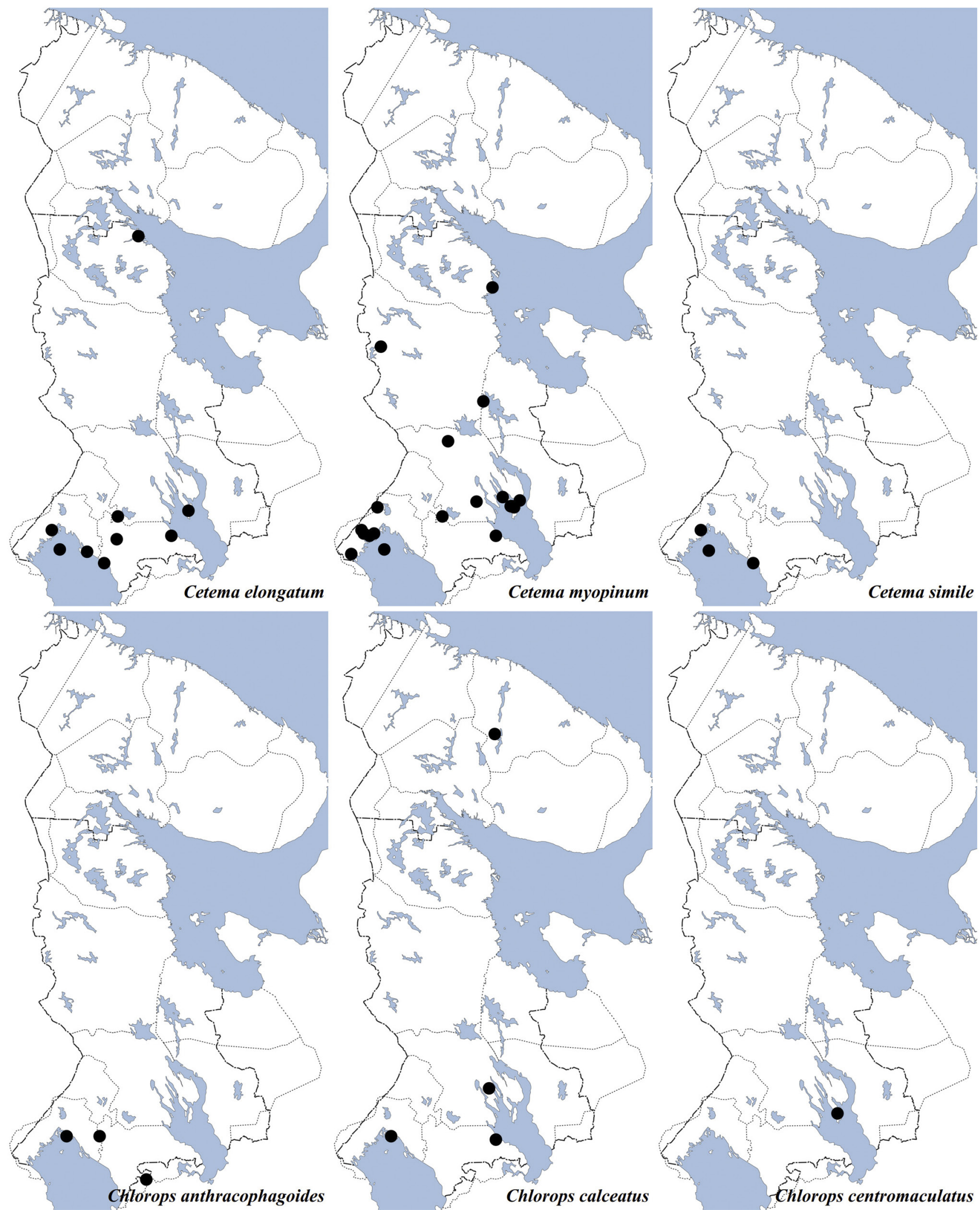
Appendix 2. Continued.
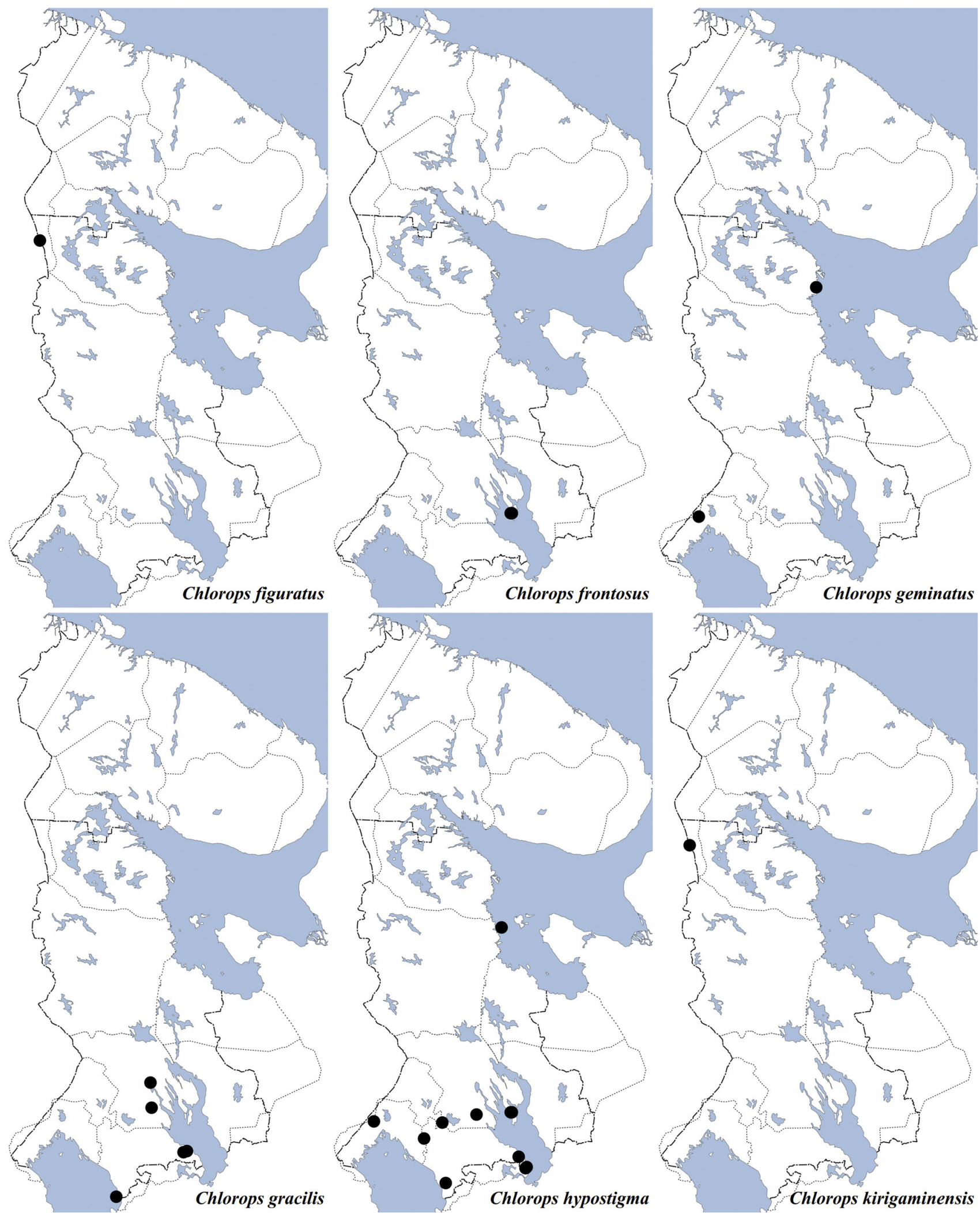
Appendix 2. Continued.
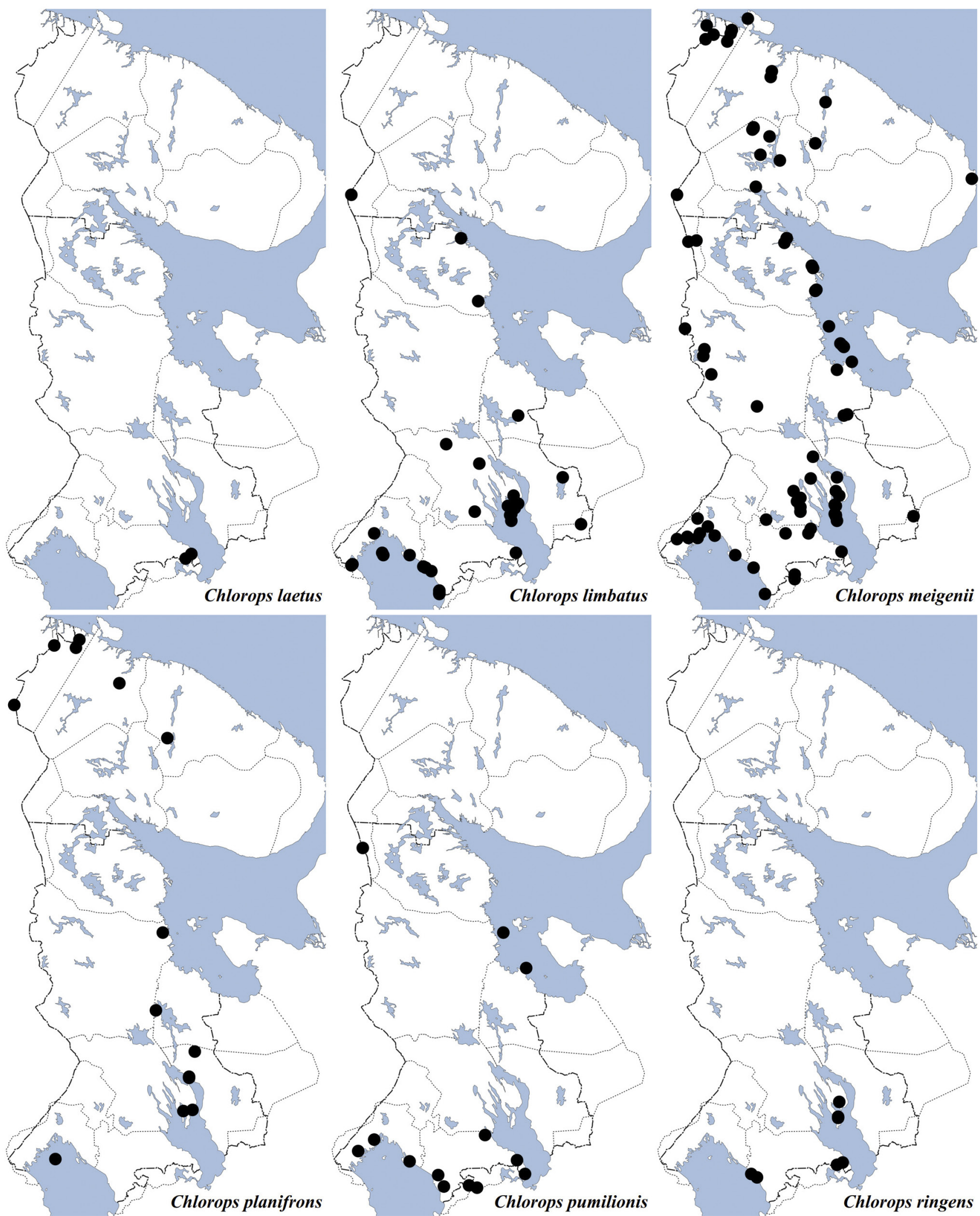
Appendix 2. Continued.
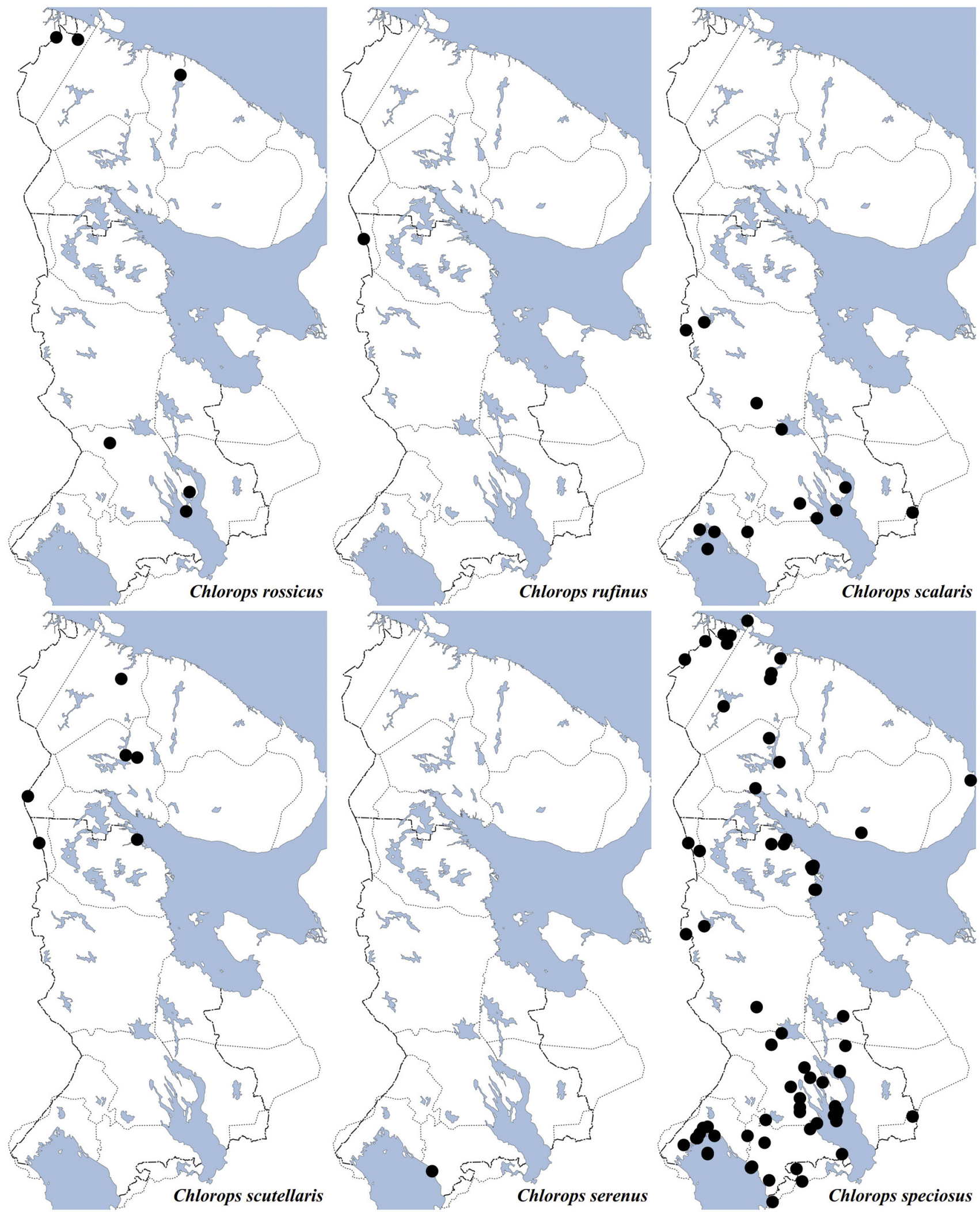
Appendix 2. Continued.
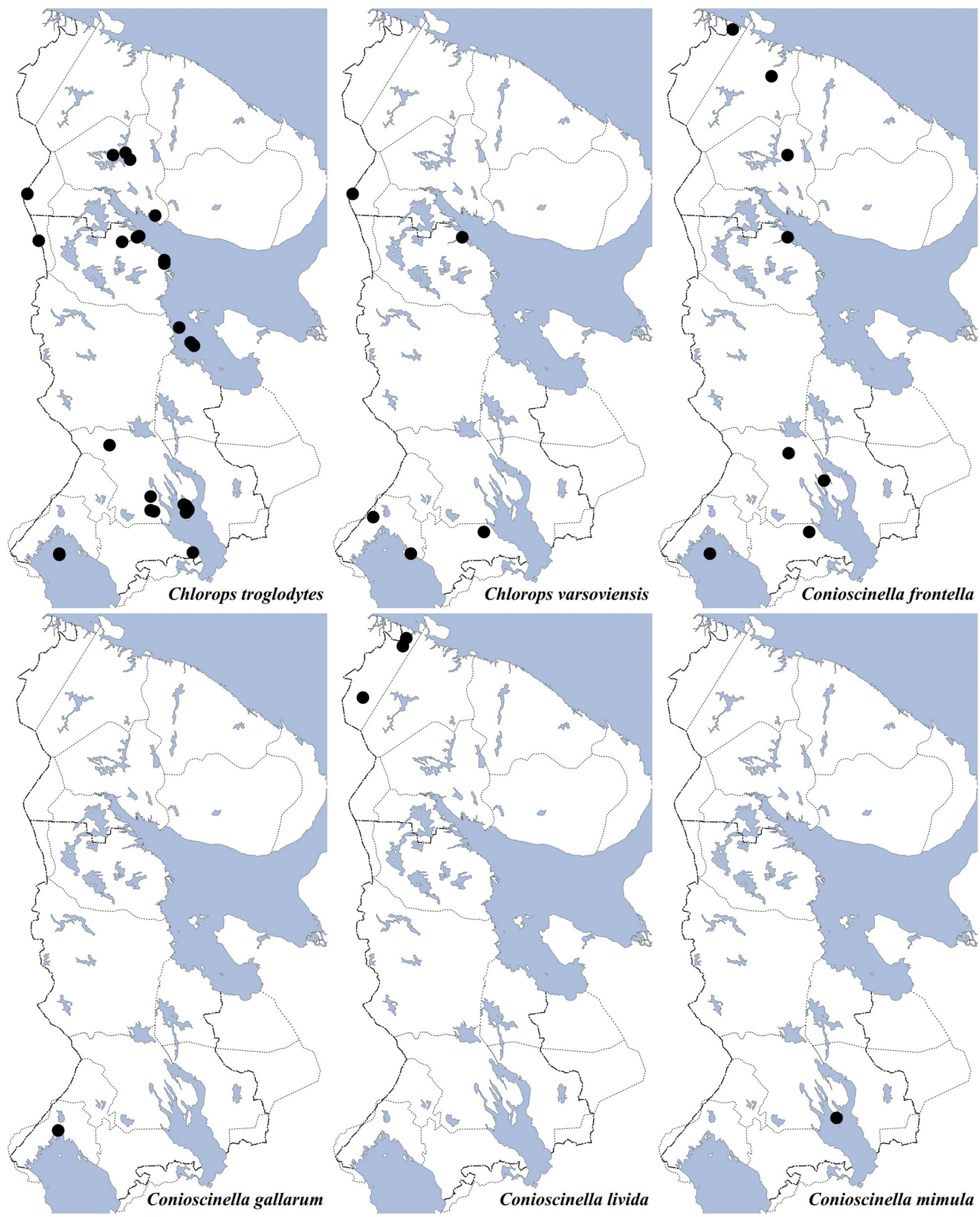
Appendix 2. Continued.
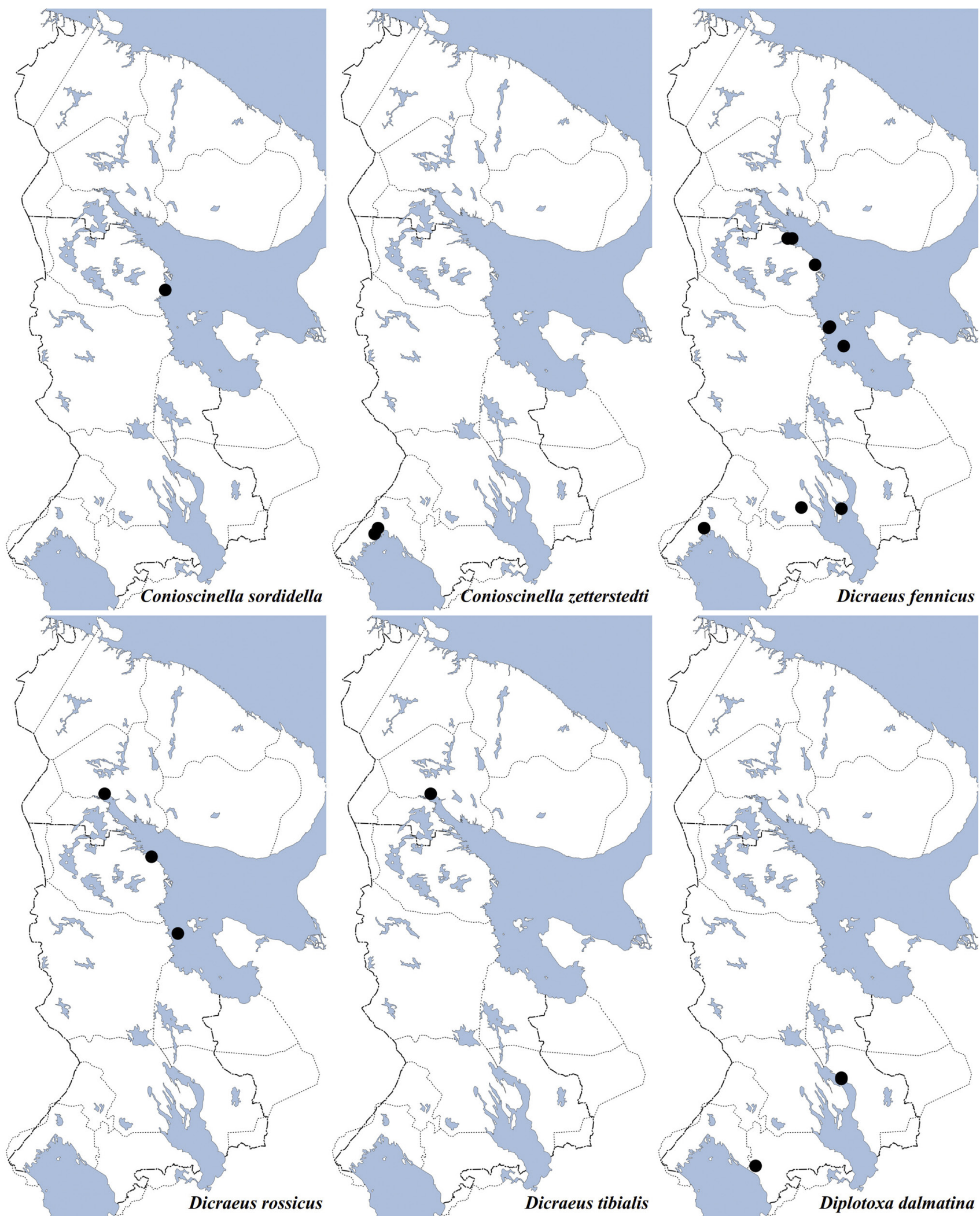
Appendix 2. Continued.
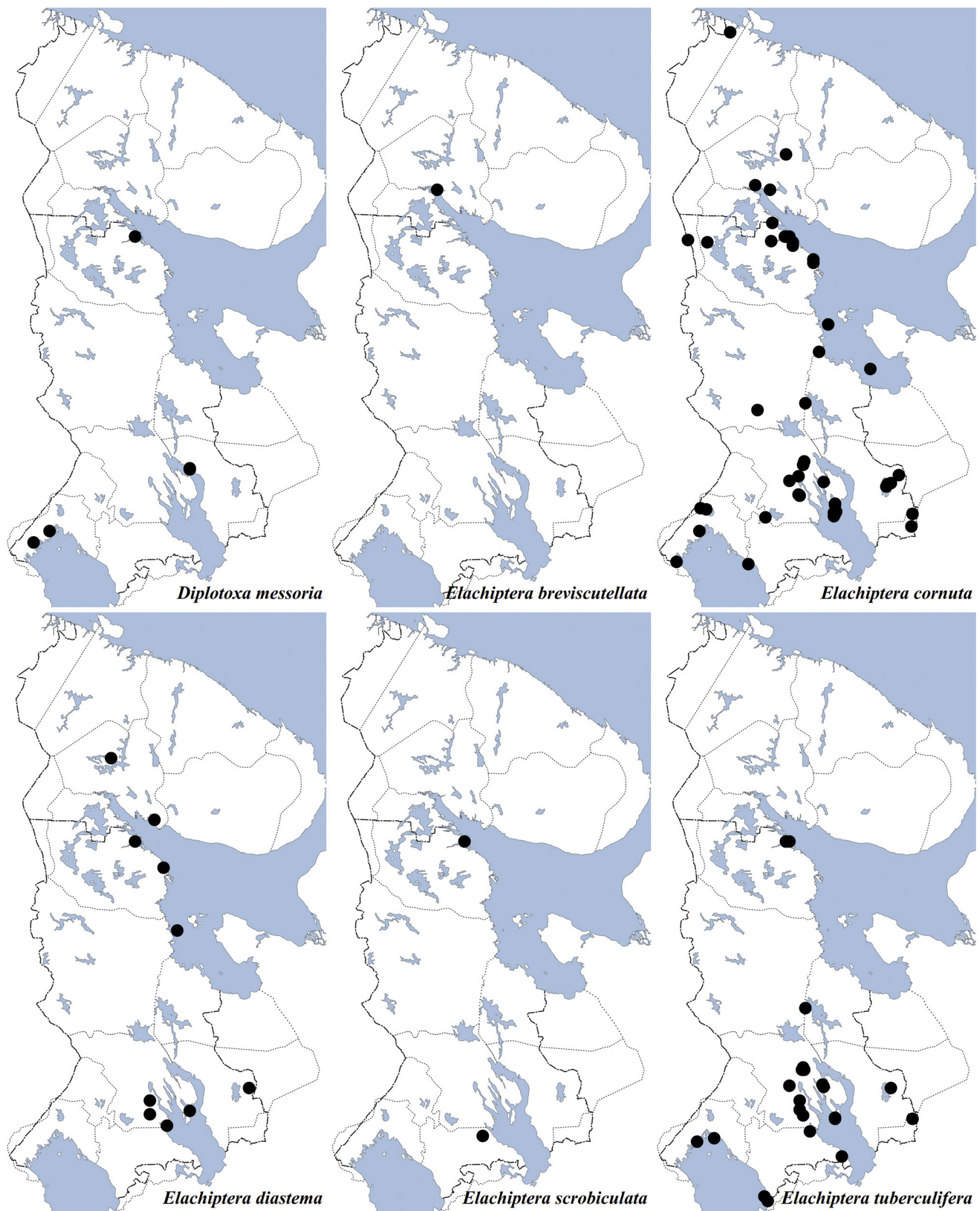
Appendix 2. Continued.
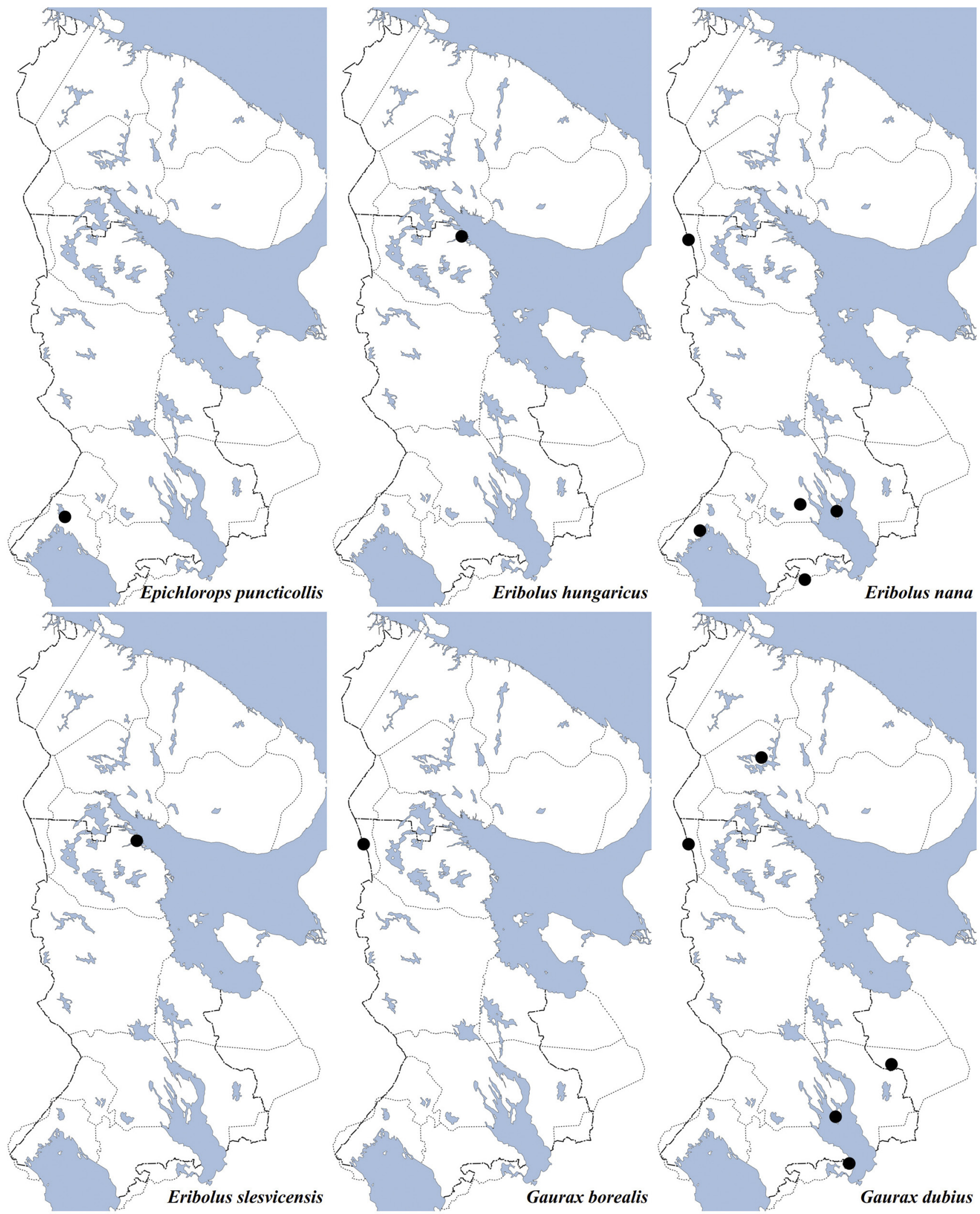
Appendix 2. Continued.
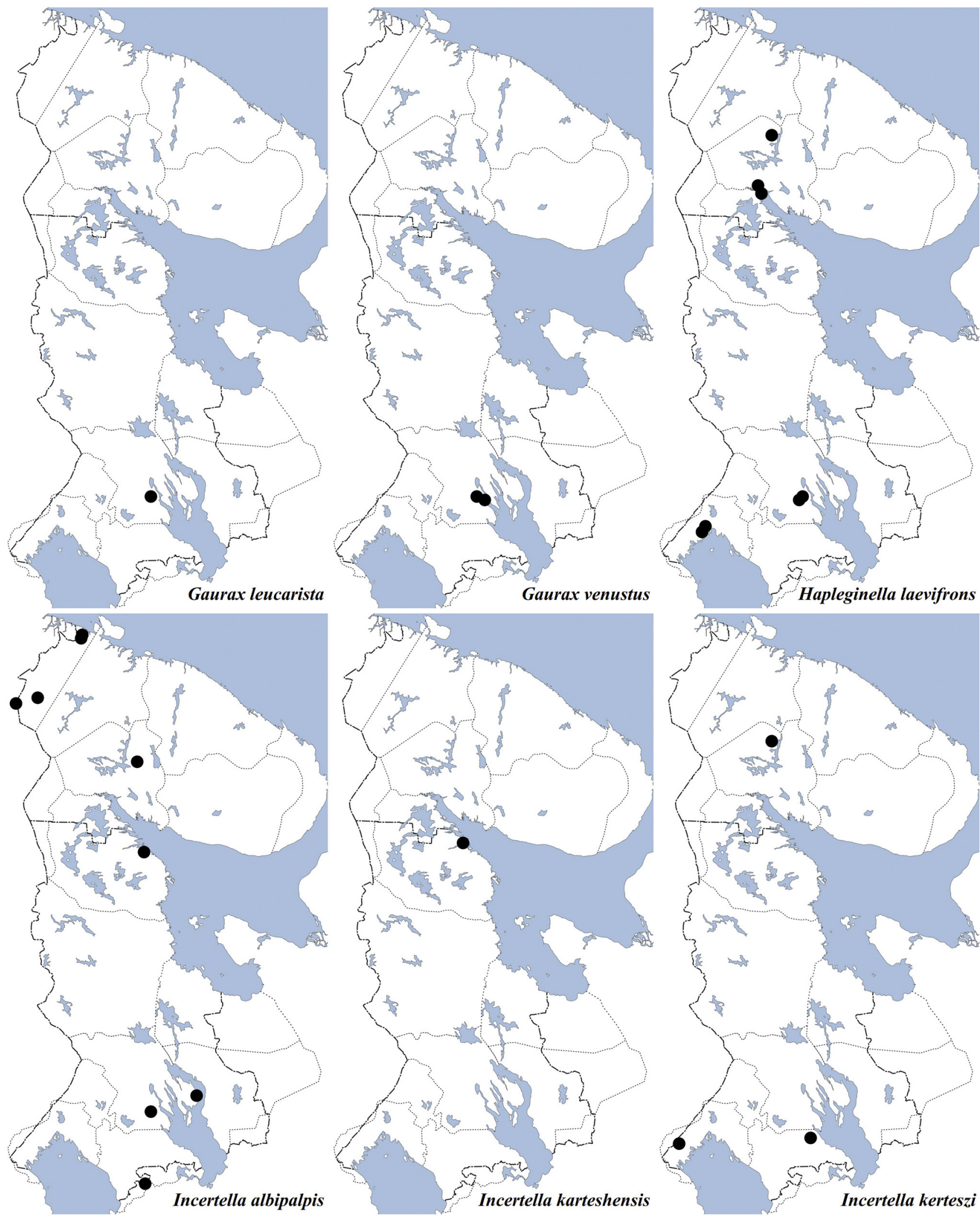
Appendix 2. Continued.
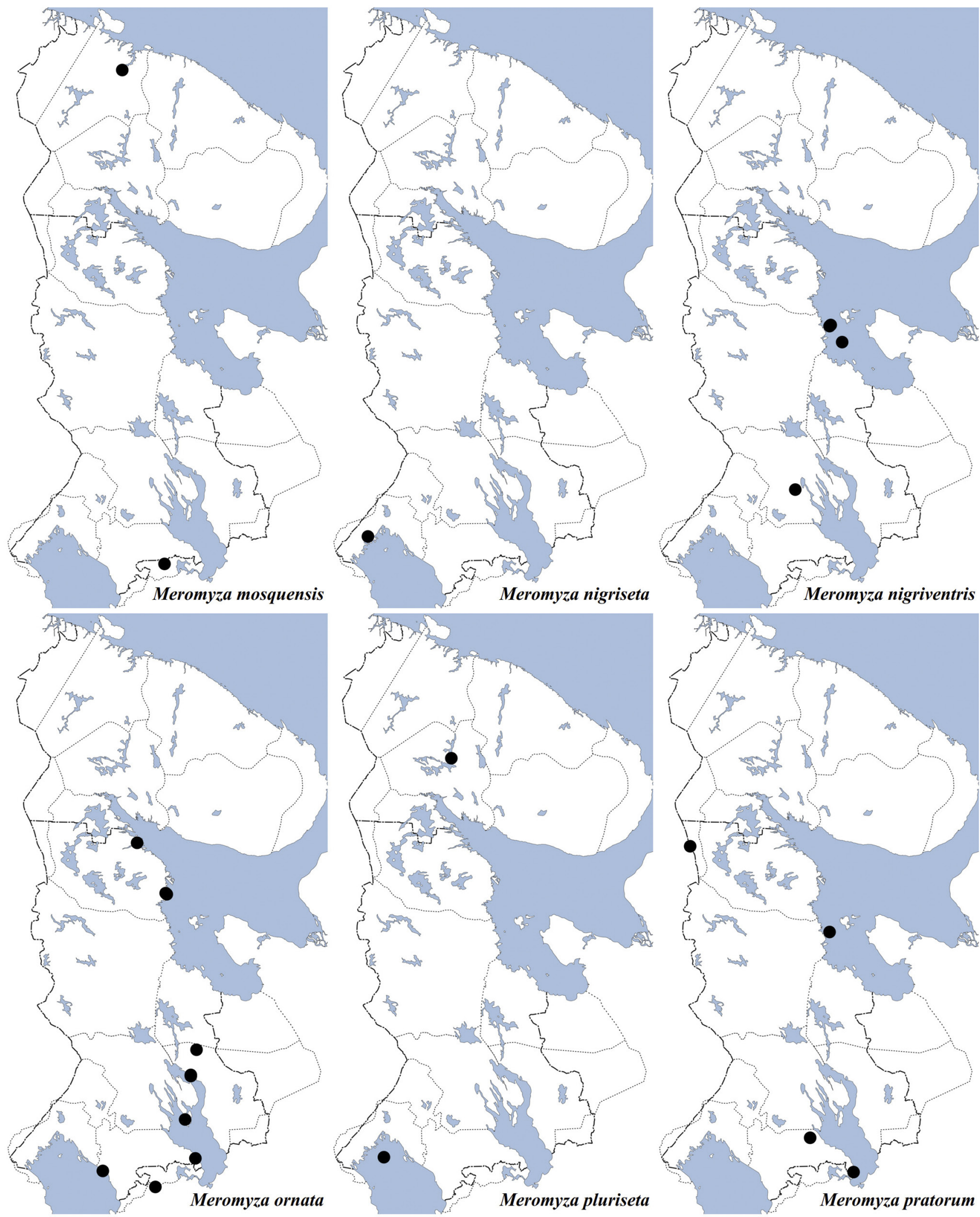
Appendix 2. Continued.
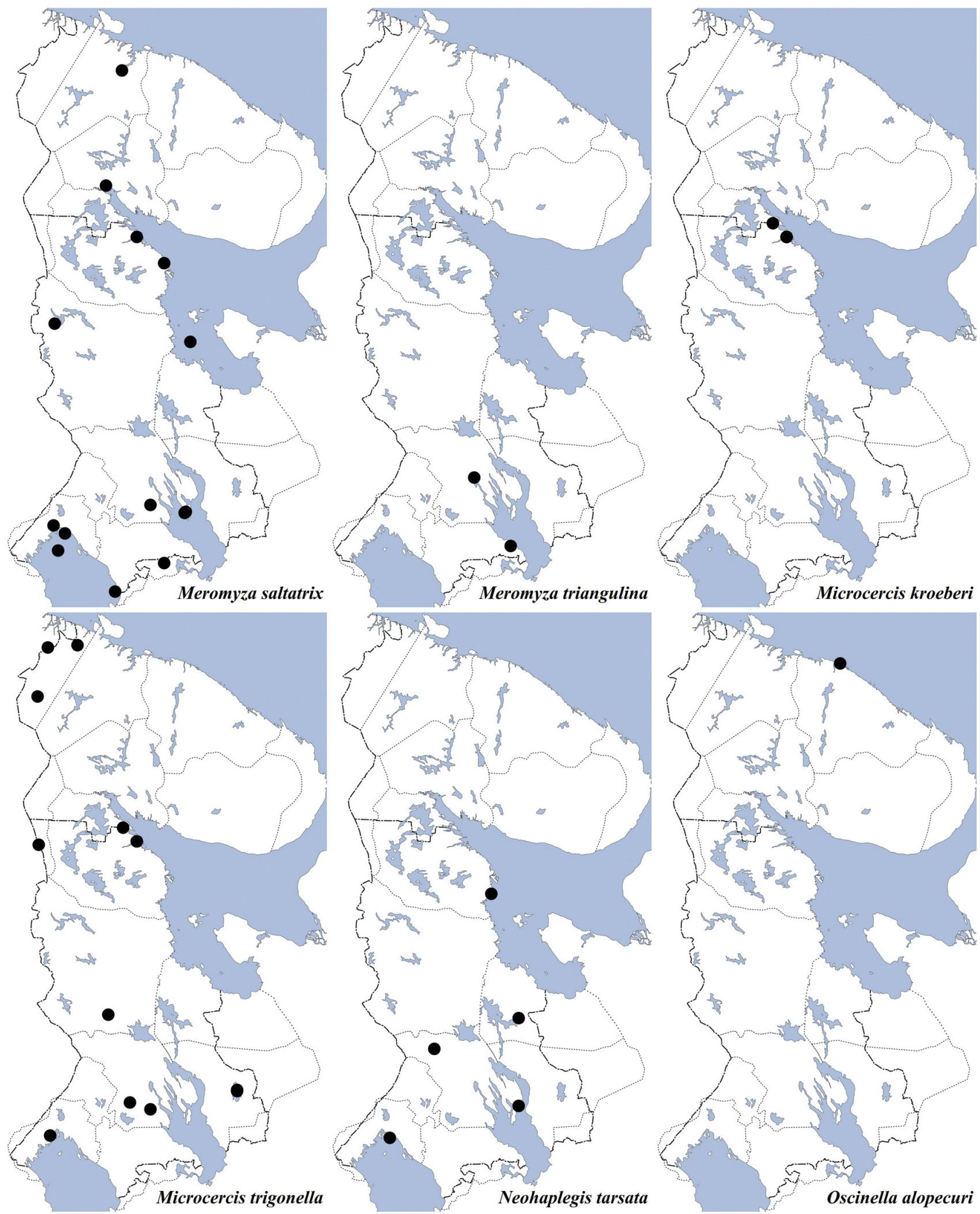
Appendix 2. Continued.
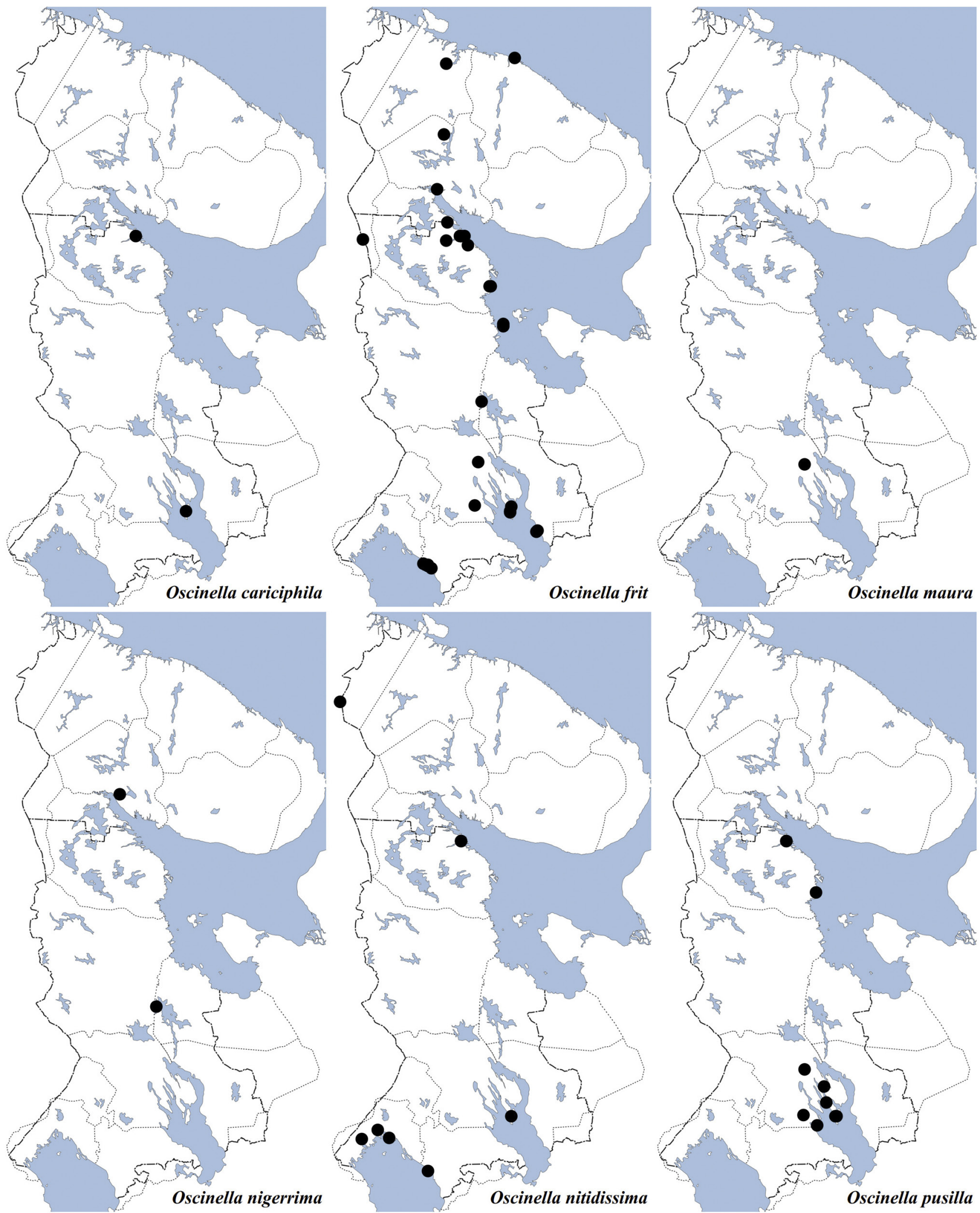
Appendix 2. Continued.
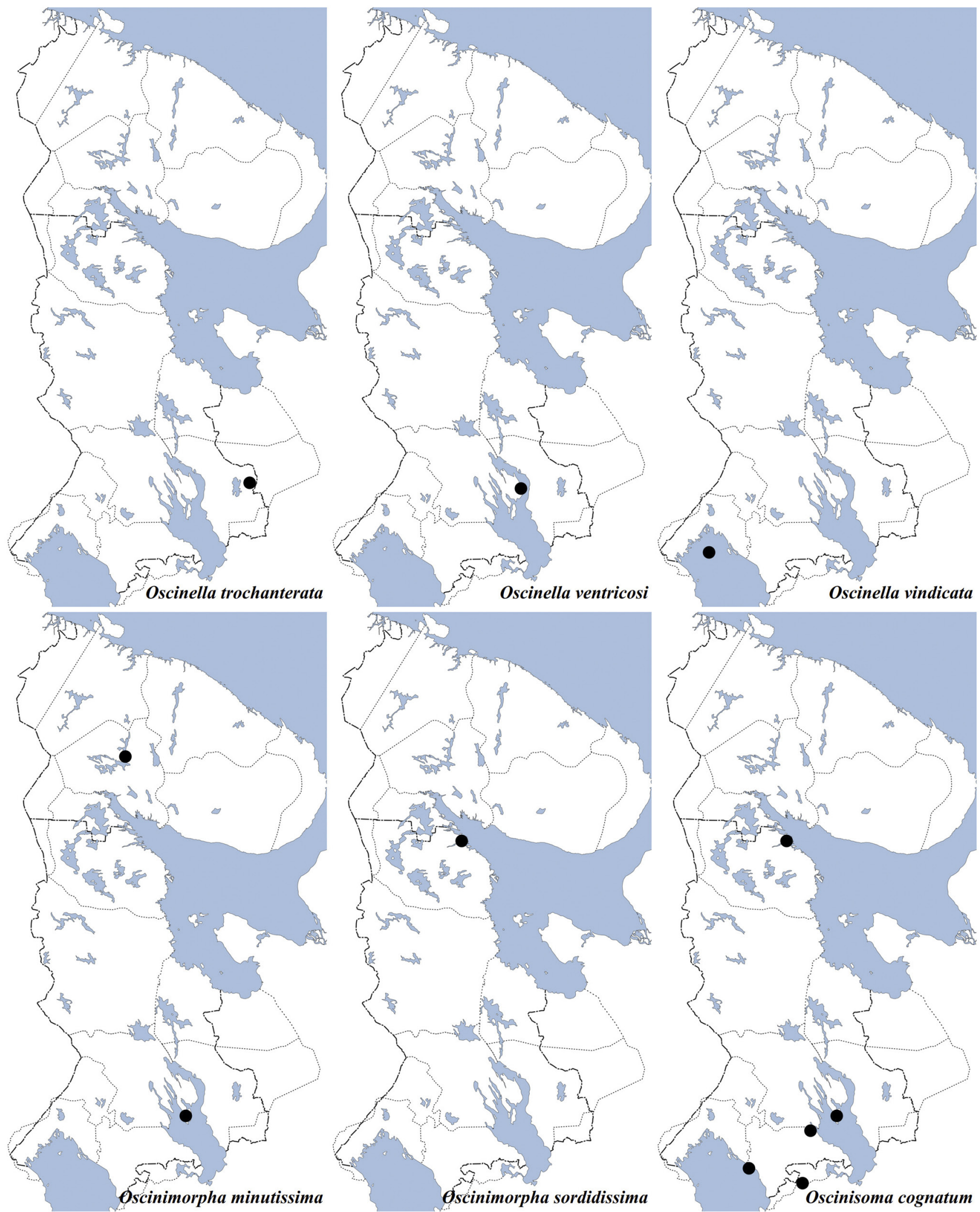
Appendix 2. Continued.
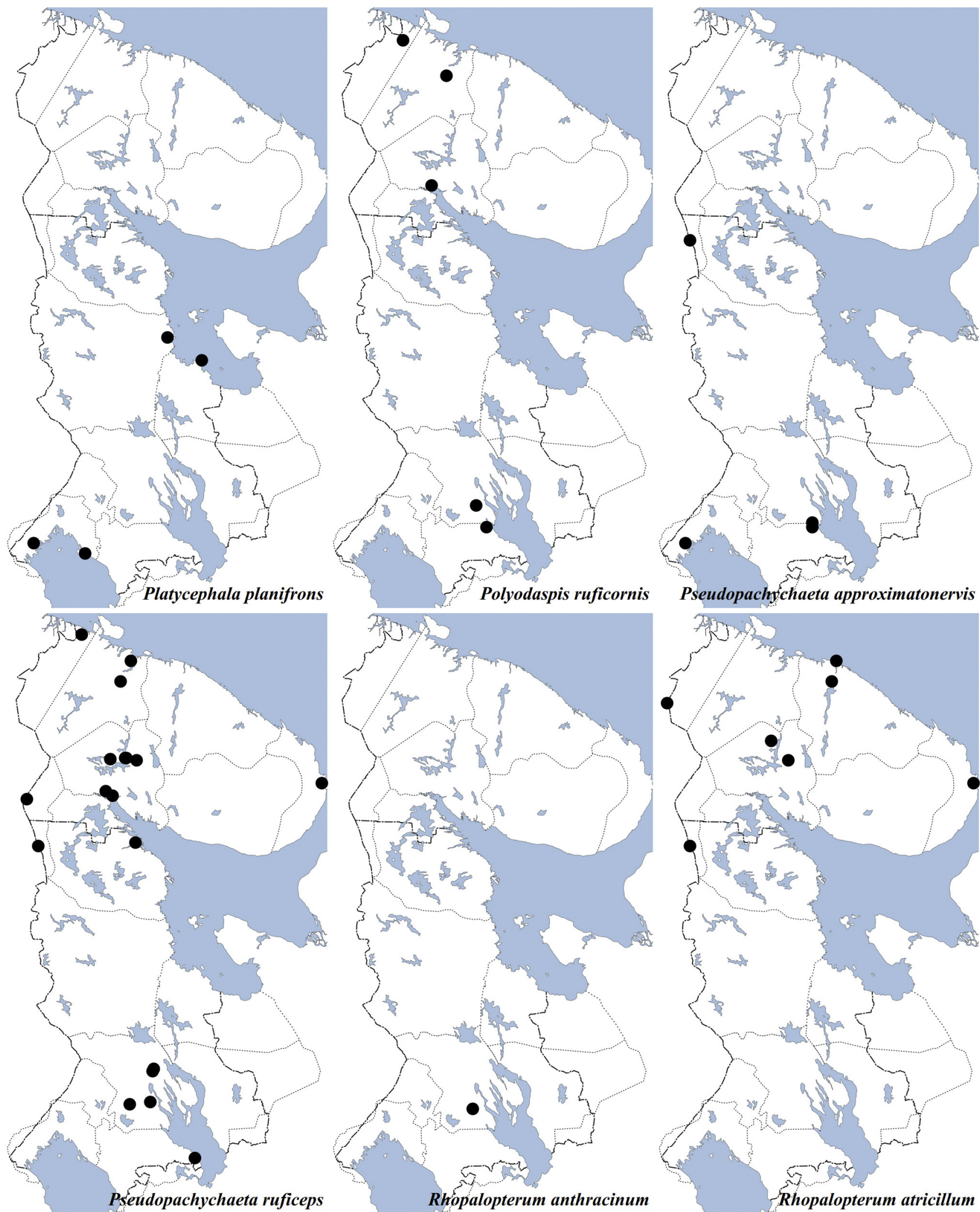
Appendix 2. Continued.
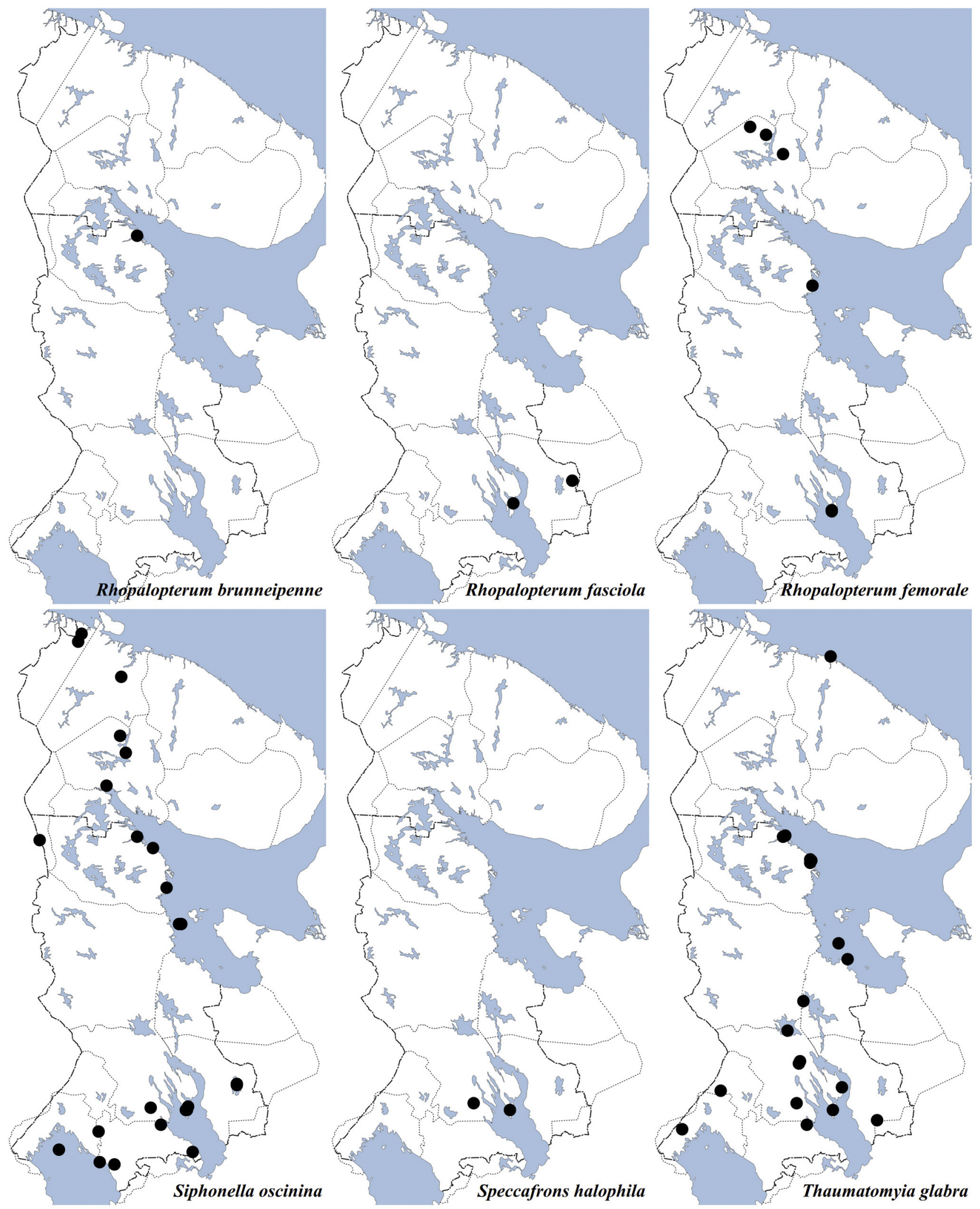
Appendix 2. Continued.
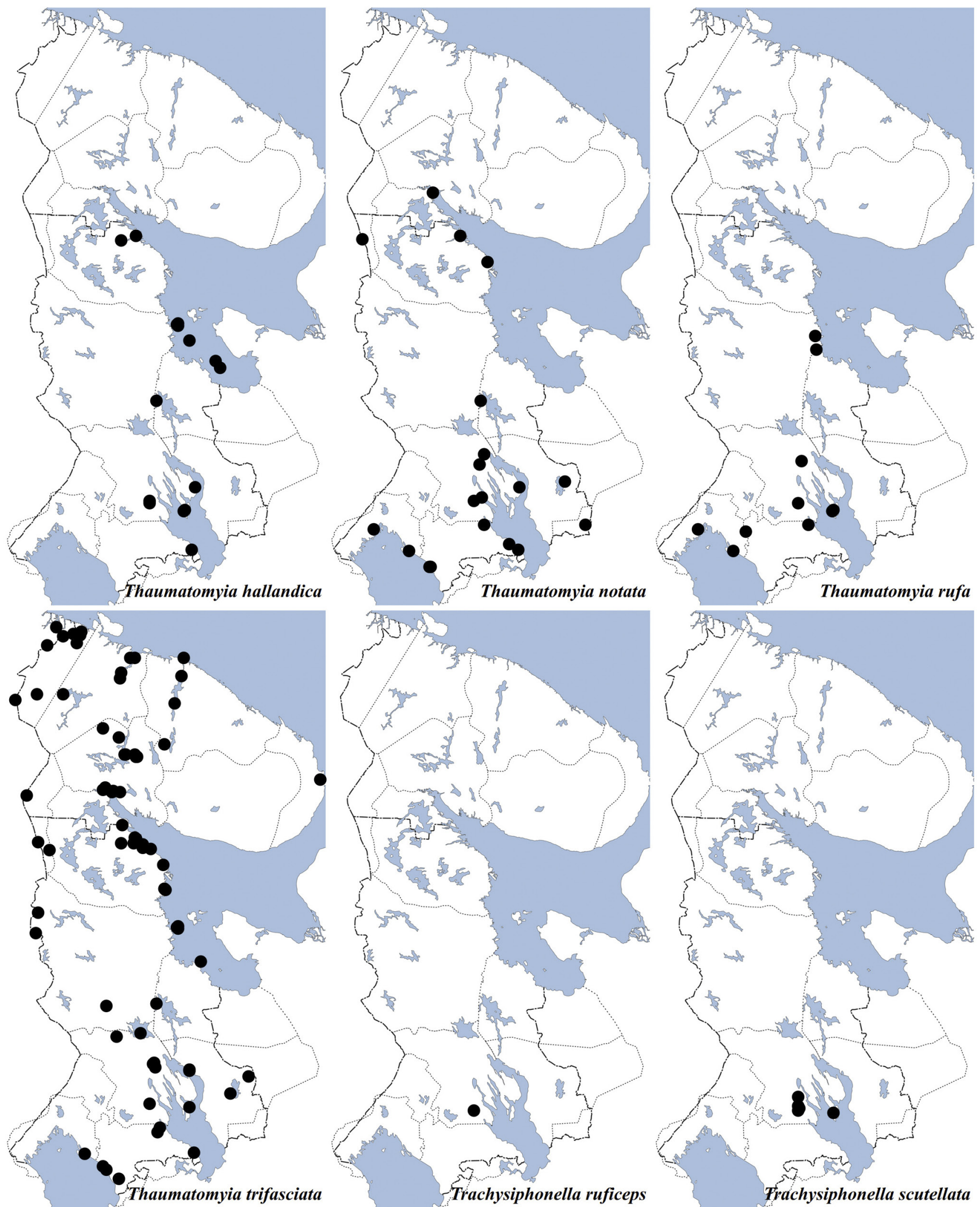
Appendix 2. Continued.

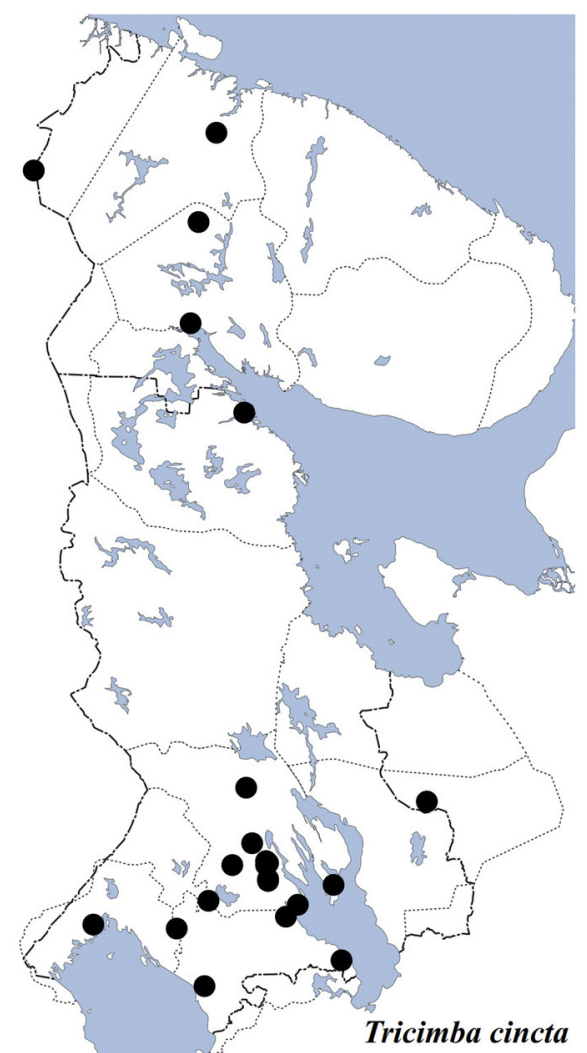

